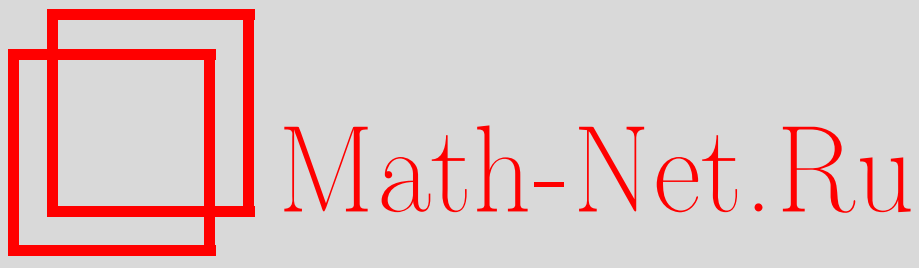

А. С. Кривошеев, Регулярность роста системы функций и системы неоднородных уравнений свертки в выпуклых областях комплексной плоскости, Изв. РАН. Сер. матем., 2000, том 64, выпуск 5, 69-132

DOI: https://doi.org/10.4213/im305

Использование Общероссийского математического портала Math-Net.Ru подразумевает, что вы прочитали и согласны с пользовательским соглашением

http://www.mathnet.ru/rus/agreement

Параметры загрузки:

IP : 54.174 .149 .18

26 апреля 2023 г., 17:36:44 
УДК 517.5

\author{
А. С. Кривошеев
}

\title{
Регулярность роста системы функций и системы неоднородных уравнений свертки в выпуклых областях комплексной плоскости
}

\begin{abstract}
В работе вводится понятие регулярности роста системы целых функций конечного порядка и типа. Это понятие является прямым и естественным обобщением классической полной регулярности роста целой функции. Получены достаточные и необходимые условия разрешимости системы неоднородных уравнений свертки в выпуклых областях комплексной плоскости. Эти условия формулируются в терминах регулярности роста системы функций, которая состоит из преобразований Лапласа аналитических функционалов, порождающих уравнения свертки. В случае гладких выпуклых областей условия разрешимости являются критерием.
\end{abstract}

Библиографиия: 18 наименований.

\section{§1. Введение. Вспомогательные сведения}

Пусть $D$ - выпуклая область в $\mathbb{C}$. Через $H(D)$ обозначим пространство функций, аналитических в $D$, с топологией равномерной сходимости на компактных подмножествах из $D ; H^{*}(D)$ - сильно сопряженное к $H(D)$ пространство, называемое пространством аналитических функционалов в области $D$. Пространство $H(D)$ является замкнутым подпространством пространства непрерывных функций в области $D$. Поэтому из теорем Хана-Банаха и Рисса следует, что каждому элементу $\mu \in H^{*}(D)$ соответствует мера $m$ с компактным носителем в $D$ такая, что

$$
(\mu, \psi)=\int_{D} \psi(y) d m(y), \quad \psi \in H(D) .
$$

Пусть $\mu$-аналитический функционал в $\mathbb{C}$. Преобразованием Лапласа функционала $\mu$ называется функция $f(z)$, определяемая равенством

$$
f(z)=\left(\mu_{y}, \exp (y z)\right), \quad z \in \mathbb{C} .
$$

Она является целой и имеет экспоненциальньй тип (т.е. порядок меньше единицы либо порядок один и конечный тип; см. [1]). Пусть $K$ - сопряженная диаграмма функции $f[1]$. Тогда для каждой окрестности $U$ компакта $K$ найдется постоянная $C_{U}$ такая, что

$$
|(\mu, \psi)| \leqslant C_{U} \sup _{z \in U}|\psi(z)|, \quad \psi \in H(\mathbb{C}) .
$$

Работа подготовлена при поддержке грантов Российского фонда фундаментальных исследований № 96-01-00095 и ведущих научных школ № 96-15-96196.

(C) А.С. Кривошеев, 2000 
Компакт $K$, обладающий этим свойством, назьвают определяющим множеством функционала $\mu$. Сопряженная диаграмма функции $f$ является наименьшим выпуклым компактом, определяюшим $\mu$. Иногда такой компакт называют носителем функционала $\mu$. Поскольку целые функции плотны в любом пространстве $H(\Omega)$, где $\Omega$ - вьпуклая область в $\mathbb{C}$, то из указанного вьше неравенства легко следует, что функционал $\mu$ продолжается как линейный и непрерывный на пространство $H(K)$ функций, аналитических на $K$. Следовательно, если некоторый сдвиг $K+z$ компакта $K$ лежит в области $D$, то для любой функции $\psi \in H(D)$ определено значение функционала $\mu$ на элементе $\psi(y+z), y \in D-z$, пространства $H(D-z) \subset H(K)$.

Пусть $D$ представляется в виде $G+K$, где $G$-выпуклая область. Тогда в силу сказанного на пространстве $H(D)$ определен оператор $\mathbf{M}$, называемый оператором свертки и действуюший по правилу:

$$
\mathbf{M}[\psi](z)=(\mu, \psi(y+z))=\int \psi(y+z) d m(y), \quad \psi \in H(D), \quad z \in G .
$$

Нетрудно видеть, что значениями оператора $\mathbf{M}$ являются функции, аналитические в области $G$, т.е. М действует из $H(D)$ в $H(G)$. При этом преобразование Лапласа $f$ функционала $\mu$ называется характеристической функиией оператора $\mathbf{M}$. Частными случаями операторов свертки являются линейные дифференциальные операторы с постоянными коэффициентами, линейные разностные и дифференциально-разностные операторы с постоянными коэффициентами как конечного, так и бесконечного порядков, некоторые типы интегральных операторов и др. (см. [2]).

Пусть $G$-выпуклая область в $\mathbb{C}, \mu_{1}, \ldots, \mu_{n}$ - аналитические функционалы в $\mathbb{C}$, $f_{1}, \ldots, f_{n}$ и $K_{1}, \ldots, K_{n}$ - соответственно их преобразования Лапласа и носители (сопряженные диаграммы). Положим

$D=G+K_{1}+\cdots+K_{n}, \quad G_{i}=G+K_{1}+\cdots+K_{i-1}+K_{i+1}+\cdots+K_{n}, \quad i=1, \ldots, n$.

Тогда для каждого $i=1, \ldots, n$ функционал $\mu_{i}$ определяет оператор свертки $\mathbf{M}_{i}$, действуюший из пространства $H(D)$ в пространство $H\left(G_{i}\right)$. Рассмотрим систему сверточных уравнений

$$
\mathbf{M}_{1}[\psi]=g_{1}, \quad \ldots, \quad \mathbf{M}_{n}[\psi]=g_{n} .
$$

Естественно возникает вопрос об условиях разрешимости этой системы. Для случая $n=1$, т.е. одного сверточного уравнения, имеется большое количество работ отечественных и зарубежных математиков, посвяшенных проблеме разрешимости. Полное ее решение в выпуклых областях комплексного пространства произвольного числа измерений найдено автором в работах [3] и [4], где имеется также список наиболее важных публикаций по этой теме. Значительно хуже обстоит дело с проблемой разрешимости систем неоднородных уравнений свертки. Здесь можно отметить работы [5] и [6], где изучались системы дифференциальных уравнений конечного порядка с постоянными коэффициентами. В работах [5], [2], [7] исследовалась разрешимость системы (1.1) в случае, когда $G$ - это вся плоскость (или $m$-мерное комплексное пространство) и функции $f_{1}, \ldots, f_{n}$ не имеют обших нулей. 
Полное решение для этого случая найдено в работе [2]. Здесь показано, что система (1.1) разрешима в $H\left(\mathbb{C}^{m}\right)$ при любой допустимой (этот термин мы разъясним несколько ниже) правой части $g=\left(g_{1}, \ldots, g_{n}\right) \in H\left(\mathbb{C}^{m}\right) \times \cdots \times H\left(\mathbb{C}^{m}\right)$ тогда и только тогда, когда существуют постоянные $a, b>0$ такие, что выполнено неравенство

$$
\left|f_{1}(z)\right|+\cdots+\left|f_{n}(z)\right| \geqslant a \exp (-b|z|), \quad z \in \mathbb{C}^{m} .
$$

K настоящему моменту разрешимость систем уравнений свертки общего вида исследована лишь в этом достаточно частном случае. Такое сушественное различие в степени изученности проблемы разрешимости одного и нескольких сверточных уравнений обусловлено возникновением массы новых сложных задач при исследовании систем уравнений. Одной из основных трудностей здесь является отсутствие приемлемой характеристики роста системы целых функций $\left(f_{1}, \ldots, f_{n}\right)$ как единого целого. В случае одного сверточного уравнения роль такой характеристики (и не только в вопросе разрешимости) прекрасно сыграло классическое понятие полной регулярности роста целой функции [1]. Оказалось, что для области $G$ с гладкой гранищей сюръективность оператора свертки $\mathbf{M}: H(G+$ $\left.K_{1}\right) \rightarrow H(G)$ эквивалентна полной регулярности роста преобразования Лапласа $f_{1}$ функционала $\mu_{1}$. Для изучения систем уравнений свертки американской школой математиков (Л. Эренпрайс, К. А. Беренстейн, Б. А. Тейлор) было введено и усиленно разрабатывается в настояшее время многими зарубежными ученьми понятие медленного убывания системы целых функций. Однако в общем случае это понятие является достаточно сложным и громоздким. Кроме того, оно малоэффективно при исследовании систем неоднородных уравнений свертки и дает некоторые результаты лишь при изучении пространств решений систем однородных сверточных уравнений.

В настоящей работе вводится новое понятие регулярности роста системы целых функций, которое является прямым и естественным обобщением классического понятия полной регулярности роста одной целой функции. На этой основе приводятся достаточные условия разрешимости системы сверточных уравнений в выпуклых областях из $\mathbb{C}$ в общем случае (т.е. при наличии общих нулей у $f_{1}, \ldots, f_{n}$ ). Показывается также, что в ряде случаев эти условия являются и необходимыми, т.е. дают критерий разрешимости системы (1.1). Например, для области $G$ с гладкой границей разрешимость (1.1) оказывается эквивалентной регулярности роста системы функций $\left(f_{1}, \ldots, f_{n}\right)$. Таким образом, вводимое здесь понятие регулярности роста систем целых функций играет ту же роль для систем уравнений свертки, что и классическое понятие полной регулярности роста целой функции для одного сверточного уравнения.

Приведем теперь некоторые известные факты и понятия, необходимые нам в дальнейшем. Пусть области $D, G, G_{i}$, функционалы $\mu_{i}$, функции $f_{i}$ и операторы $\mathbf{M}_{i}-$ те же, что и выше. Положим $\mathbf{M}=\left(\mathbf{M}_{1}, \ldots, \mathbf{M}_{n}\right)$ и

$$
\mathbf{M}[\psi]=\left(\mathbf{M}_{1}[\psi], \ldots, \mathbf{M}_{n}[\psi]\right) .
$$

Оператор $\mathbf{M}$ действует из $H(D)$ в произведение $H\left(G_{1}\right) \times \cdots \times H\left(G_{n}\right)$. Пусть $\mathbf{M}^{*}-$ сопряженный к $\mathbf{M}$ оператор, действуюший из $H^{*}\left(G_{1}\right) \times \cdots \times H^{*}\left(G_{n}\right)$ в $H^{*}(D)$. Известно (см. [1], [2]), что преобразование Лапласа устанавливает алгебраический и 
топологический изоморфизм пространств $H^{*}(D)$ и $P_{D}$, где $P_{D}-$ пространство целых функций экспоненциального типа, сопряженные диаграммы которых лежат в области $D$. Топология в $P_{D}$ задается как топология индуктивного предела пространств

$$
P_{j}=\left\{\varphi \in H(\mathbb{C}): \sup _{z \in \mathbb{C}}|\varphi(z)| \exp \left[-H_{L_{j}}(z)\right]<+\infty\right\}
$$

где $L_{j}$ - последовательность выпуклых компактов из области $D$ такая, что $L_{j} \subset$ int $L_{j+1}$ и $\cup L_{j}=D ; H_{L}-$ опорная функция множества $L$ :

$$
H_{L}(y)=\sup _{z \in L} \operatorname{Re}(z y), \quad y \in \mathbb{C} .
$$

На самом деле функция $H_{L}$ является опорной для множества, комплексно сопряженного к $L$. Однако в последнее время в комплексном анализе для удобства $H_{L}$ называют опорной функиией именно множества $L$. Так будем называть ее и мы. Отметим, что опорная функция ограниченного множества всегда непрерывна. Если же выпуклое множество неограничено, то его опорная функция полунепрерывна снизу.

Из определения топологии в $P_{D}$ следует (см. [2]), что последовательность функций $\left\{\varphi_{k}\right\} \subset P_{D}$ сходится в $P_{D}$ к функции $\varphi$, если найдется номер $j$ такой, что $\varphi,\left\{\varphi_{k}\right\} \subset P_{j}$ и $\left\{\varphi_{k}\right\}$ сходится к $\varphi$ в пространстве $P_{j}$. Сходимость же в $P_{j}$ означает, что для некоторого $C>0$ и всех $k$ выполнено неравенство $\left|\varphi_{k}(z)\right| \leqslant$ $C \exp H_{L_{j}}(z), z \in \mathbb{C}$, и $\left\{\varphi_{k}\right\}$ сходится равномерно на каждом компакте из $\mathbb{C}$ к функции $\varphi$.

При указанном выше изоморфизме оператор $\mathbf{M}^{*}$ однозначно определяет некоторый оператор, действующий из $P_{G_{1}} \times \cdots \times P_{G_{n}}$ в $P_{D}$, который мы обозначим $\Sigma$. Пусть $\nu=\left(\nu_{1}, \ldots, \nu_{n}\right) \in H^{*}\left(G_{1}\right) \times \cdots \times H\left(G_{n}\right)$ и $\mu=\mathbf{M}^{*}[\nu]$. Обозначим через $\varphi_{i}$ и $F$ преобразования Лапласа соответственно функционалов $\nu_{i}$ и $\mu$. Найдем явньй вид оператора $\Sigma$. Для этого в равенство, определяющее сопряженный оператор, подставим экспоненту и получим

$$
\begin{aligned}
F(z) & =\left(\mathbf{M}^{*}[\nu], \exp (z \xi)\right)=(\mathbf{M}[\exp (z \xi)], \nu)=\sum_{i=1}^{n}\left(\left(\mu_{i}, \exp ((y+\xi) z)\right), \nu_{i}\right) \\
& =\sum_{i=1}^{n}\left(\left(\mu_{i}, \exp (z \xi)\right) \exp (y z), \nu_{i}\right)=\sum_{i=1}^{n} f_{i}(z)\left(\exp (y z), \nu_{i}\right)=\sum_{i=1}^{n} f_{i}(z) \varphi_{i}(z) .
\end{aligned}
$$

Таким образом, для каждого вектора $\varphi=\left(\varphi_{1}, \ldots, \varphi_{n}\right) \in P\left(G_{1}\right) \times \cdots \times P\left(G_{n}\right)$ имеем

$$
\Sigma[\varphi](z)=f_{1}(z) \varphi_{1}(z)+\cdots+f_{n}(z) \varphi_{n}(z)
$$

Пусть $g \in H\left(G_{1}\right) \times \cdots \times H\left(G_{n}\right)$ лежит в образе оператора $\mathbf{M}$, т.е. $\mathbf{M}[h]=g$ для некоторого $h \in H(D)$. Если $\nu$ - произвольный функционал из ker $\mathbf{M}^{*}$, то

$$
(\nu, g)=(\nu, \mathbf{M}[h])=\left(M^{*}[\nu], h\right)=0 .
$$

Следовательно, необходимым условием разрешимости системы (1.1) с правой частью $g=\left(g_{1}, \ldots, g_{n}\right)$ является равенство $(\nu, g)=0$ для любого $\nu \in \operatorname{ker} \mathbf{M}^{*}$. С учетом явного вида оператора $\Sigma$ в терминах преобразования Лапласа это условие 
можно переписать следующим образом: $(\nu, g)=0$ для каждого $\nu=\left(\nu_{1}, \ldots, \nu_{n}\right)$ такого, что $f_{1} \varphi_{1}+\cdots+f_{n} \varphi_{n} \equiv 0\left(\varphi_{1}, \ldots, \varphi_{n}-\right.$ те же, что и выше). Правую часть $g$ системы (1.1), удовлетворяюшую указанному условию, будем называть допустимой. Очевидно, множество всех допустимых правых частей является замкнутым подпространством в $\prod H\left(G_{i}\right)$. Сказанное означает, что ставить вопрос о разрешимости системы (1.1) имеет смысл лишш для элементов этого подпространства.

ЛЕмма 1.1. Образ оператора $\mathbf{M}$ плотен во множестве допустимых правых частей системь (1.1).

ДокАЗАТЕЛЬСтво. Рассмотрим систему вектор-функций

$$
\Theta=\{\exp (z \lambda) f(\lambda)\}, \quad \lambda \in \mathbb{C}
$$

где $f=\left(f_{1}, \ldots, f_{n}\right)$. Эта система лежит в im М. Действительно,

$$
\mathbf{M}[\exp y \lambda]=\left(\mu_{y}, \exp (y+z) \lambda\right)=\exp (z \lambda) f(\lambda)
$$

Здесь $\mu=\left(\mu_{1}, \ldots, \mu_{n}\right)$. Образ оператора $\mathbf{M}$ содержит также и линейные комбинации функций из $\Theta$. Достаточно показать теперь, что линейная оболочка $\Theta$ плотна во множестве допустимых правых частей системы (1.1). Пусть $\nu=\left(\nu_{1}, \ldots, \nu_{n}\right)-$ произвольный функционал из $H^{*}\left(G_{1}\right) \times \cdots \times H^{*}\left(G_{n}\right)$, обращающийся в нуль на элементах системы $\Theta$. Тогда

$$
0=(\nu, \exp (z \lambda) f(\lambda))=\sum_{i=1}^{n}\left(\nu_{i}, \exp (z \lambda)\right) f_{i}(\lambda)=\sum_{i=1}^{n} \varphi_{i}(\lambda) f_{i}(\lambda), \quad \lambda \in \mathbb{C}
$$

где $\varphi_{i}$ - преобразование Лапласа $\nu_{i}$. Согласно сказанному выше из этого равенства вытекает, что $(\nu, g)=0$ для любой допустимой правой части $g$. По следствию из теоремы Хана-Банаха это означает, что линейная оболочка $\Theta$ плотна во множестве допустимых правых частей (1.1). Лемма доказана.

ЗАмЕчАнИЕ 1.1. Из доказанной леммы следует, что система (1.1) разрешима в $H(D)$ при любой допустимой правой части $g \in \prod H\left(G_{i}\right)$ тогда и только тогда, когда образ оператора $\mathbf{M}$ замкнут. Так как $H(D)$ и $\prod H\left(G_{i}\right)$ являются полными метризуемыми пространствами [2], т.е. пространствами Фреше, то по теореме о сопряженной операции для таких пространств (см. [8]) замкнутость im M равносильна замкнутости образа сопряженного оператора $\mathbf{M}^{*}$, или, что то же самое, замкнутости множества im $\Sigma \subset P_{D}$.

Пусть $\Lambda=\left\{\lambda_{k}, m_{k}\right\}$, где $\left\{\lambda_{k}\right\}$ - последовательность общих нулей $f_{1}, \ldots, f_{n}$ и $m_{k}-$ кратность $\lambda_{k}$, т.е.

$$
\frac{d^{m} f_{i}}{d z^{m}}\left(\lambda_{k}\right)=0, \quad 0 \leqslant m<m_{k}, \quad k=1,2, \ldots, \quad i=1, \ldots, n,
$$

и для каждого $k=1,2, \ldots$ найдется номер $i=1, \ldots, n$ такой, что $\frac{d^{m_{k}} f_{i}}{d z^{m}}\left(\lambda_{k}\right) \neq 0$. Нетрудно показать (см., например, [2]), что система функций

$$
\left\{z^{m} \exp \left(z \lambda_{k}\right), \quad 0 \leqslant m<m_{k}, \quad k=1,2, \ldots\right\}
$$


принадлежит ядру оператора $\mathbf{M}$. Говорят, что подпространство ker $\mathbf{M}$ допускает спектральный синтез, если линейная оболочка системы (1.2) плотна в нем. Согласно [9], [10] достаточным условием для наличия спектрального синтеза в подпространстве ker $\mathbf{M}$ является сушествование такой функции $F \in P_{D}$, что для каждого $i=1, \ldots, n$ функция $F / f_{i}$ целая. В рассматриваемом здесь случае в качестве $F$ можно взять $\exp (y z) f_{1}(z) \ldots f_{n}(z)$, где $y$-произвольная фиксированная точка области $G$. Таким образом, подпространство $\operatorname{ker} \mathbf{M}$ в нашей ситуации всегда допускает спектральный синтез. В пространстве $P_{D}$ определим множество

$$
I(D, \Lambda)=\left\{\psi \in P_{D}: \frac{d^{m} \psi}{d z^{m}}\left(\lambda_{k}\right)=0, \quad 0 \leqslant m<m_{k}, \quad k=1,2, \ldots\right\} .
$$

Оно, очевидно, замкнуто по отношению к линейным операциям. Оно также замкнуто в топологии пространства $P_{D}$, поскольку сходимость в $P_{D}$ мажорирует сходимость на компактных подмножествах из $\mathbb{C}$. Следовательно, $I(D, \Lambda)$ является замкнутым подпространством в $P_{D}$.

В заключение этого параграфа приведем лемму, которая сводит задачу разрешимости системы (1.1) к проблеме специального представления функций из подпространства $I(D, \Lambda)$.

ЛЕмма 1.2. Следующие два утверждения эквивалентны.

1) Для каждой функиии $F \in I(D, \Lambda)$ существует әлемент $\left(\varphi_{1}, \ldots, \varphi_{n}\right)$ пространства $P_{G_{1}} \times \cdots \times P_{G_{n}}$ такой, что

$$
F(z)=\varphi_{1}(z) f_{1}(z)+\cdots+\varphi_{n}(z) f_{n}(z) \quad \forall z \in \mathbb{C} .
$$

2) Система уравнений свертки (1.1) разрешима в пространстве $H(D)$ при любой допустимой правой части $g \in H\left(G_{1}\right) \times \cdots \times H\left(G_{n}\right)$.

ДокАзАТЕльСтво. 1) $\rightarrow$ 2). Как отмечено вьше, для вьполнения 2) достаточно установить замкнутость множества $\mathrm{im} \Sigma$. Очевидно, что имеет место включение $\operatorname{im} \Sigma \subset I(D, \Lambda)$. С другой стороны, выполнение 1$)$ означает, что $I(D, \Lambda) \subset \operatorname{im} \Sigma$. Таким образом, $\operatorname{im} \Sigma=I(D, \Lambda)$. Требуемое утверждение следует теперь из замкнутости множества $I(D, \Lambda)$.

$2) \rightarrow 1)$. Нужно показать, что $I(D, \Lambda) \subset \operatorname{im} \Sigma$. Если выполнено 2$)$, то в силу замечания, сделанного вьше, подпространство im $\Sigma$ замкнуто. Предположим, что $I(D, \Lambda) \backslash \operatorname{im} \Sigma$ не пусто. Возьмем произвольный функционал $\nu \in H^{*}(D)$, преобразование Лапласа $\psi(z)$ которого лежит в $I(D, \Lambda) \backslash \operatorname{im} \Sigma$. В силу рефлексивности пространства $H(D)$ (см., например, [2]) с учетом теоремы Хана-Банаха найдем функцию $h \in H(D)$ такую, что

$$
(\nu, h) \neq 0 \quad \text { и } \quad(\tau, h)=0 \quad \forall \tau \in \operatorname{im} \mathbf{M}^{*} .
$$

Пусть $s$ - произвольный элемент пространства $H^{*}\left(G_{1}\right) \times \cdots \times H^{*}\left(G_{n}\right)$. Тогда

$$
(s, \mathbf{M}[h])=\left(\mathbf{M}^{*}[s], h\right)=0 .
$$

Следовательно, $h \in \operatorname{ker} \mathrm{M}$. С другой стороны, так как $\psi \in I(D, \Lambda)$, то легко видеть (см. [2]), что функционал $\nu$ обращается в нуль на любой функции из системы (1.2). Поскольку, как показано выше, подпространство ker $\mathbf{M}$ допускает спектральньй синтез, то отсюда следует, что $\nu$ аннулирует ker M. В частности, $(\nu, h)=0$. Полученное противоречие завершает доказательство. 


\section{§2. Регулярность роста системы целых функций конечного порядка и типа}

В этом параграфе мы введем новое понятие, характеризующее совместное асимптотическое поведение системы целых функций, и опишем некоторые его свойства. Это понятие мы введем, последовательно обобщая классическое понятие полной регулярности роста целой функции.

Пусть $\psi(z)$ - целая функция порядка (не выше) $\rho>0$ и конечного типа (при порядке $\rho$ ), т.е. для некоторых $a, b>0$ выполнено неравенство

$$
|\psi(z)| \leqslant a \exp \left(b|z|^{\rho}\right) \quad \forall z \in \mathbb{C}
$$

Через $h_{\psi}(z)$ обозначим индикатор функции $\psi(z)$ :

$$
h_{\psi}(z)=\varlimsup_{t \rightarrow+\infty} \frac{\ln |\psi(t z)|}{t^{\rho}}, \quad z \in \mathbb{C} .
$$

Функция $h_{\psi}(z)$ субгармонична, положительно однородна порядка $\rho$ (т.е. $h_{\psi}(t z)=$ $\left.t^{\rho} h_{\psi}(z), z \in \mathbb{C}, t \geqslant 0\right)$ и непрерывна в $\mathbb{C}($ см. [1]).

Приведем классическое определение регулярности роста. Говорят [1], что $\psi(z)$ имеет (вполне) регулярный рост вдоль луча (на луче) $t y, t>0$, если регулярный рост имеет субгармоническая функция $\ln |\psi|$, т.е. если

$$
\lim _{t \rightarrow+\infty, t \notin E_{y}} \frac{\ln |\psi(t y)|}{t^{\rho}}=h_{\psi}(y),
$$

где $E_{y}-$ множество нулевой относительной меры, т.е. $\operatorname{mes}\left[E_{y} \cap(0, r)\right] \rightarrow 0$ при $r \rightarrow+\infty$.

Пусть $\mathbb{S}$ - единичная окружность с центром в нуле и $z \in \mathbb{S}$. Через $\mathbf{E}(z, \delta)$, $\delta>0$, обозначим совокупность последовательностей $\left\{z_{k}\right\}_{k=1}^{\infty}$, лежащих на луче $\{t z, t>0\}$ и удовлетворяющих условиям $\left|z_{k}\right| \rightarrow+\infty$ и $\left|z_{k+1}\right| /\left|z_{k}\right|<1+\delta$, $k=1,2, \ldots$ Кроме того, пусть $\mathbf{E}(z)$ обозначает множество последовательностей $\left\{z_{k}\right\}_{k=1}^{\infty}$, лежаших на луче $\{t z, t>0\}$ и таких, что для каждого $\delta>0$ и некоторого $k(\delta)$ последовательность $\left\{z_{k}\right\}_{k=k(\delta)}^{\infty}$ принадлежит $\mathbf{E}(z, \delta)$.

В настоящее время сушествует достаточно много различных эквивалентных указанному выше определений регулярности роста целой функции. Приведем на наш взгляд самое простое из них.

ОПРЕДЕЛЕНИЕ 2.1 [11], [12]. Говорят, что функция $\psi(z)(\ln |\psi|)$ имеет регулярньй рост вдоль луча $t y, y \in \mathbb{S}$, если существует последовательность $\left\{z_{k}\right\} \in \mathbf{E}(y)$ такая, что

$$
\lim _{k \rightarrow \infty} \frac{\ln \left|\psi\left(z_{k}\right)\right|}{\left|z_{k}\right|^{\rho}}=h_{\psi}(y)
$$

Одним из наиболее важных достоинств регулярности роста является то, что ее наличие у характеристической функции оператора свертки необходимо и достаточно для разрешимости сверточного уравнения. В связи с этим обобщение этого понятия на случай системы целых функций можно было бы считать содержательным, если оно будет играть аналогичную роль для систем сверточных уравнений. 
Однако ни одно из указанных вьше определений не приспособлено для такого обобщения. Поэтому мы дадим еще одно, эквивалентное предыдущим, определение регулярности роста.

Пусть $\Lambda=\left\{\lambda_{j}, m_{j}\right\}_{j=1}^{\infty}$, где $\left\{\lambda_{j}\right\}_{j=1}^{\infty} \subset \mathbb{C}$ и $\left\{m_{j}\right\}_{j=1}^{\infty}-$ последовательность натуральных чисел. Для каждых $\delta>0$ и $0 \neq x \in \mathbb{C} \backslash\left\{\lambda_{j}\right\}_{j=1}^{\infty}$ введем функцию

$$
q_{\delta}(z, x, \Lambda)=\prod_{\lambda_{j} \in B(x, \delta|x|)}\left(\frac{z-\lambda_{j}}{x-\lambda_{j}}\right)^{m_{j}}, \quad z \in \mathbb{C},
$$

где $B(x, r)$ - круг с центром в точке $x$ и радиуса $r>0$. Если же $B(x, \delta|x|)$ не содержит точек из последовательности $\left\{\lambda_{j}\right\}$, то положим $q_{\delta}(z, x, \Lambda) \equiv 1$. Легко видеть, что верны следуюшие соотношения:

$$
q_{\delta}(x, x, \Lambda)=1, \quad\left|q_{\delta}(z, x, \Lambda)\right| \geqslant 1, \quad z \notin B(x, 2 \delta|x|) .
$$

Пусть $\Lambda_{\psi}=\left\{\lambda_{j}, m_{j}\right\}_{j=1}^{\infty}-$ последовательность нулей и их кратностей целой функции $\psi(z)$.

ОПРЕДЕЛЕнИЕ 2.2. Будем говорить, что целая функция $\psi(z)$ порядка (не выше) $\rho$ и конечного типа (при порядке $\rho$ ) имеет регулярный рост вдоль луча ty, $y \in \mathbb{S}$, если существует последовательность $\left\{z_{k}\right\} \in \mathbf{E}(y)$, удовлетворяющая условию: для каждых $r \in(0,1)$ и $\varepsilon>0$ найдутся $\delta(r, \varepsilon)>0$ и номер $k_{0}$ такие, что при любом положительном $\delta<\delta(r, \varepsilon)$ выполнены неравенства

$$
\ln \left|\frac{\psi(z)}{q_{\delta}\left(z, z_{k}, \Lambda_{\psi}\right)}\right| \geqslant h_{\psi}(z)-\varepsilon|z|^{\rho}, \quad z \in B\left(z_{k}, r \delta\left|z_{k}\right|\right), \quad k \geqslant k_{0}
$$

\section{ПРЕДЛОЖЕНИЕ 2.1. Определения 2.1 и 2.2 әквивалентны.}

ДоКАЗАТЕЛЬСТво. Из определения индикатора $h_{\psi}$ и первого соотношения в (2.2) следует, что неравенство (2.3) влечет за собой выполнение (2.1). Докажем теперь обратное. Предположим, что выполнено (2.1). Фиксируем произвольные числа $\tilde{\varepsilon}>0$ и $r \in(0,1)$. Из непрерывности $h_{\psi}(z)$ и теоремы Хартогса о семействе субгармонических функций легко следует (см., например, [2]), что найдутся $R, \delta^{\prime}>0$, для которых имеет место неравенство

$$
\frac{\ln |\psi(t z)|}{t^{\rho}} \leqslant h_{\psi}(y)+\tilde{\varepsilon}, \quad z \in B\left(y, 6 \delta^{\prime}\right), \quad t>R .
$$

В силу непрерьвности функции $h_{\psi}(z)$ (уменьшая при необходимости $\delta^{\prime}>0$ ) можно считать, что

$$
\left|h_{\psi}(y)-h_{\psi}(z)\right|<\tilde{\varepsilon}, \quad z \in B\left(y, \delta^{\prime}\right) .
$$

Согласно (2.1) выберем номер $k_{0}$, удовлетворяюший условиям: $\left|z_{k}\right|>R$ и

$$
\frac{\ln \left|\psi\left(z_{k}\right)\right|}{\left|z_{k}\right|^{\rho}} \geqslant h_{\psi}(y)-\tilde{\varepsilon}, \quad k \geqslant k_{0}
$$


Для каждого $k \geqslant k_{0}$ рассмотрим функцию

$$
\psi_{k}(z)=\psi(z)\left[\psi\left(z_{k}\right) q_{\delta}\left(z, z_{k}, \Lambda_{\psi}\right)\right]^{-1}, \quad \delta \in\left(0, \delta^{\prime}\right) .
$$

По построению $\psi_{k}$ - целая функция и $\psi_{k}\left(z_{k}\right)=1$. Из соотношений $(2.2),(2.4),(2.6)$ и принципа максимума модуля легко следует оценка

$$
\ln \left|\psi\left(z_{k}\right)\right| \leqslant 2 \tilde{\varepsilon}\left|z_{k}\right|^{\rho}, \quad z \in B\left(z_{k}, 6 \delta\left|z_{k}\right|\right) .
$$

Отсюда по теореме об оценке снизу модуля аналитической функции (см. [1, с. 33]) находим, что в круге $B\left(z_{k}, \delta\left|z_{k}\right|\right)$, но вне исключительных кружков с общей суммой радиусов, равной $(1-r) \delta\left|z_{k}\right| / 2$, выполнено неравенство

$$
\ln \left|\psi_{k}(z)\right| \geqslant-2 a \tilde{\varepsilon}\left|z_{k}\right|^{\rho},
$$

где $a=2+\ln (3 e / 2 \eta), \eta=(1-r) / 8$. При этом, как видно из доказательства леммы Картана об оценке снизу полинома, на которой основана цитируемая теорема, каждый из исключительных кружков содержит хотя бы один нуль функции $\psi_{k}(z)$. По построению $\psi_{k}$ не имеет нулей в круге $B\left(z_{k}, \delta\left|z_{k}\right|\right)$. Поэтому $B\left(z_{k}, r \delta\left|z_{k}\right|\right)$ не пересекает исключительных кружков. Тогда в силу $(2.5),(2.6)$, однородности $h_{\psi}$, определения $\psi_{k}$ и последнего неравенства получаем

$$
\ln \left|\frac{\psi(z)}{q_{\delta}\left(z, z_{k}, \Lambda_{\psi}\right)}\right| \geqslant\left[h_{\psi}(z)-(1+2 a) \tilde{\varepsilon}|z|^{\rho}\right](1 \pm \delta)^{\rho}, \quad z \in B\left(z_{k}, r \delta\left|z_{k}\right|\right),
$$

где знак внутри круглой скобки совпадает со знаком выражения, стоящего в квадратной скобке. Полагая $\varepsilon=2(1+a) \tilde{\varepsilon}$ и выбирая $\delta(r, \varepsilon)$ (в зависимости также от величин $\rho$ и $\left.\max _{z \in \mathbb{S}}\left|h_{\psi}(z)\right|<\infty\right)$ достаточно малым, при любом положительном $\delta<\delta(r, \varepsilon)$ получаем оценку (2.3). Предложение доказано.

Понятие регулярности роста в той форме, которая присутствует в определении 2.2 , можно естественным образом распространить на случай системы целых функций.

ОПРЕДЕЛЕНИЕ 2.3. Будем говорить, что система целых функций $\left(\psi_{1}, \ldots, \psi_{n}\right)$ порядка (не вьше) $\rho$ и конечного типа (при порядке $\rho$ ) имеет регулярный рост вдоль луча ty $(y \in \mathbb{S})$, если сушествует последовательность $\left\{z_{k}\right\} \in \mathbf{E}(y)$, удовлетворяюшая условию: для каждых $r \in(0,1)$ и $\varepsilon>0$ найдутся $\delta(r, \varepsilon)>0$ и номер $k_{0}$ такие, что при любом $\delta \in(0, \delta(r, \varepsilon))$ вьполнены неравенства

$$
\max _{1 \leqslant i \leqslant n}\left[\ln \left|\frac{\psi_{i}(z)}{q_{\delta}\left(z, z_{k}, \Lambda\right)}\right|-h_{\psi_{i}}(z)\right] \geqslant-\varepsilon|z|^{\rho}, \quad z \in B\left(z_{k}, r \delta\left|z_{k}\right|\right), \quad k \geqslant k_{0},
$$

где $\Lambda=\left\{\lambda_{j}, m_{j}\right\}_{j=1}^{\infty}-$ последовательность всех обших нулей и их кратностей системы $\left(\psi_{1}, \ldots, \psi_{n}\right)$.

Простое сравнение определений 2.2 и 2.3 показывает, что понятие регулярности роста системы функций $\left(\psi_{1}, \ldots, \psi_{n}\right)$ в случае $n=1$ совпадает с обычной регулярностью роста целой функции.

Приведем формально более слабые, чем в определении 2.3 , условия на систему функций $\left(\psi_{1}, \ldots, \psi_{n}\right)$, эквивалентные, тем не менее, регулярности роста этой системы. 
ПРЕДЛОЖЕНИЕ 2.2. Пусть $y \in \mathbb{S}$. Предположим, что выполнено следующее. Существует последовательность $\left\{z_{k}\right\}_{k=1}^{\infty} \in \mathbf{E}(y)$, удовлетворяющая условиям: для каждого $\varepsilon>0$ найдутся номер $k_{0}$, число $r_{0} \in(0,1)$ и последовательности $\left\{\delta_{k}\right\}_{k=1}^{\infty}, \quad\{i(k)\}_{k=1}^{\infty}$ такие, что $\inf _{k \geqslant 1} \delta_{k}>0, \quad i(k) \in\{1, \ldots, n\}$ $u$

$$
\begin{gathered}
\max _{1 \leqslant i \leqslant n}\left[\ln \left|\frac{\psi_{i}(z)}{q_{\delta_{k}}\left(z, z_{k}, \Lambda\right)}\right|-h_{\psi_{i}}(z)\right] \geqslant-\varepsilon|z|^{\rho}, \quad z \in B\left(z_{k}, r_{0} \delta_{k}\left|z_{k}\right|\right), \quad k \geqslant k_{0} \\
\ln \left|\psi_{i(k)}(z)\right| \leqslant h_{\psi_{i(k)}}\left(z_{k}\right)+\varepsilon\left|z_{k}\right|^{\rho}, \quad z \in B\left(z_{k}, 3 \delta_{k}\left|z_{k}\right|\right), \quad k \geqslant k_{0}, \quad \\
\ln \left|\psi_{i(k)}\left(z_{k}\right)\right| \geqslant h_{\psi_{i(k)}}\left(z_{k}\right)-\varepsilon\left|z_{k}\right|^{\rho}, \quad k \geqslant k_{0}
\end{gathered}
$$

Тогда система $\left(\psi_{1}, \ldots, \psi_{n}\right)$ имеет регулярный рост на луче ty.

ДокАЗАТЕЛЬСтво. Пусть $\tilde{\varepsilon}>0$ и $r \in(0,1)$ фиксированы. Выберем любое положительное $\varepsilon<a \tilde{\varepsilon}$, где $a=\left[1-2^{\rho+1} \ln (1-r)\right]^{-1}$. Согласно $(2.8)$ и $(2.9)$ имеем

$$
\ln \left|\frac{\psi_{i(k)}(z)}{\psi_{i(k)}\left(z_{k}\right)}\right| \leqslant 2 \varepsilon\left|z_{k}\right|^{\rho}, \quad z \in B\left(z_{k}, 3 \delta_{k}\left|z_{k}\right|\right), \quad k \geqslant k_{0} .
$$

Отсюда и из теоремы о нулях аналитической функции в круге (см. [1]) следует, что число нулей (с учетом кратности) $l(k)$ функции $\psi_{i(k)}$ в круге $B\left(z_{k}, \delta_{k}\left|z_{k}\right|\right)$ удовлетворяет оценке

$$
l(k) \leqslant 2 \varepsilon\left|z_{k}\right|^{\rho}, \quad k \geqslant k_{0} .
$$

Пусть $\delta(r, \tilde{\varepsilon})=\min \left\{r_{0} \inf _{k \geqslant 1} \delta_{k}, 1 / 2\right\}$ и $\delta \in(0, \delta(r, \tilde{\varepsilon}))$. Положим

$$
q_{k}(z)=\prod_{\lambda_{j} \in B\left(z_{k}, \delta_{k}\left|z_{k}\right|\right) \backslash B\left(z_{k}, \delta\left|z_{k}\right|\right)}\left(\frac{z-\lambda_{j}}{z_{k}-\lambda_{j}}\right)^{m_{j}},
$$

где $\Lambda=\left\{\lambda_{j}, m_{j}\right\}$ - последовательность обших нулей и их кратностей системы $\left(\psi_{1}, \ldots, \psi_{n}\right)$. Если $\left\{\lambda_{j}\right\}_{j=1}^{\infty} \cap B\left(z_{k}, \delta_{k}\left|z_{k}\right|\right) \backslash B\left(z_{k}, \delta\left|z_{k}\right|\right)=\varnothing$, то полагаем $q_{k}(z) \equiv 1$. Нетрудно видеть, что

$$
\left|q_{k}(z)\right| \geqslant(1-r)^{m(k)}, \quad z \in B\left(z_{k}, r \delta\left|z_{k}\right|\right), \quad k \geqslant k_{0},
$$

где $m(k)$ - число обших нулей (с учетом кратности) функций $\psi_{1}, \ldots, \psi_{n}$ в кольце $B\left(z_{k}, \delta_{k}\left|z_{k}\right|\right) \backslash B\left(z_{k}, \delta\left|z_{k}\right|\right)$. Так как $m(k) \leqslant l(k)$, то из предыдущего и $(2.10)$ получаем

$\ln \left|q_{k}(z)\right| \geqslant 2 \ln (1-r) \varepsilon\left|z_{k}\right|^{\rho} \geqslant 2^{\rho+1} \ln (1-r) \varepsilon|z|^{\rho}, \quad z \in B\left(z_{k}, r \delta\left|z_{k}\right|\right), \quad k \geqslant k_{0}$.

Отсюда для $k \geqslant k_{0}$, всех $i=1, \ldots, n$ и каждого $z \in B\left(z_{k}, r \delta\left|z_{k}\right|\right)$ имеем

$$
\ln \left|\frac{\psi_{i}(z)}{q_{\delta}\left(z, z_{k}, \Lambda\right)}\right|=\ln \left|\frac{\psi_{i}(z)}{q_{\delta_{k}}\left(z, z_{k}, \Lambda\right)}\right|+\ln \left|q_{k}(z)\right| \geqslant \ln \left|\frac{\psi_{i}(z)}{q_{\delta_{k}}\left(z, z_{k}, \Lambda\right)}\right|-(\tilde{\varepsilon}-\varepsilon)|z|^{\rho} .
$$

Это с учетом (2.7) и определения $\delta(r, \tilde{\varepsilon})$ дает нам требуемое для регулярности роста системы $\left(\psi_{1}, \ldots, \psi_{n}\right)$ неравенство. Предложение доказано. 
ЗАМЕчАНИЕ 2.1. Условия предложения 2.2 легко следуют из условий определения 2.3. Действительно, (2.7) и (2.9) непосредственно вытекают из неравенства в этом определении. Соотношение же (2.8), как и в (2.4), выполнено для каждой функции $\psi_{i}$ при всех достаточно больших $k$ (т.е. для всех $\delta_{k}>0$, меньших некоторого $\delta_{0}$, зависяшего от $\varepsilon$ ). Таким образом, условия предложения 2.2 дают другую эквивалентную формулировку понятия регулярности роста системы целых функций.

Рассмотрим теперь ситуацию, когда каждая из функций $\psi_{1}, \ldots, \psi_{n}$ по отдельности имеет регулярный рост на луче $t y$. В этом случае можно привести простые условия на взаимное расположение нулей этих функций, достаточные для того, чтобы система $\left(\psi_{1}, \ldots, \psi_{n}\right)$ имела регулярный рост вдоль луча $t y$. Поскольку объединение конечного числа множеств нулевой относительной меры также является множеством нулевой относительной меры, то согласно приведенному здесь классическому определению регулярности роста целых функций найдется последовательность $\left\{z_{k}\right\}_{k=1}^{\infty} \in \mathbf{E}(y)$ (единая для всех функций $\psi_{1}, \ldots, \psi_{n}$ ) такая, что

$$
\lim _{k \rightarrow \infty} \frac{\ln \left|\psi_{i}\left(z_{k}\right)\right|}{\left|z_{k}\right|^{\rho}}=h_{\psi_{i}}(y), \quad i=1, \ldots, n
$$

Как и выше, отсюда и из теоремы об оценке снизу модуля аналитической функции следует, что для $\psi_{i, k}(z)=\psi_{i}(z) q_{\delta}^{-1}\left(z, z_{k}, \Lambda\right)$ верно неравенство

$$
\ln \left|\psi_{i, k}(z)\right| \geqslant h_{\psi_{i}}(z)-\varepsilon|z|^{\rho},
$$

которое выполнено в круге $B\left(z_{k}, \delta\left|z_{k}\right|\right)$, но вне исключительного множества $C_{i, k}$, $i=1, \ldots, n, k \geqslant k_{0}$. Для регулярности роста системы $\left(\psi_{1}, \ldots, \psi_{n}\right)$ согласно определению 2.3 достаточно потребовать теперь, чтобы для каждого $r \in(0,1)$ и всех достаточно больших номеров $k$ пересечение $C_{1, k} \cap \cdots \cap C_{n, k}$ было пустым в круге $B\left(z_{k}, r \delta\left|z_{k}\right|\right)$.

В некоторых случаях можно дать довольно простое описание множеств $C_{i, k}$. Пусть $\Gamma$ - открытый угол (с вершиной в нуле), содержаший луч $\{t y, t>0\}$; $\left\{\lambda_{i, j}, m_{i, j}\right\}$ - последовательность, построенная следующим образом. Она содержит все нули $\lambda_{i, j}$ функции $\psi_{i}(z)$, лежащие в $Г$ и не являющиеся общими для $\psi_{1}, \ldots, \psi_{n}$, при этом $m_{i, j}$ обозначает кратность $\lambda_{i, j}$ как нуля $\psi_{i}(z)$. Оставшаяся (после исключения указанных $\lambda_{i, j}$ ) часть последовательности $\left\{\lambda_{i, j}\right\}_{j=1}^{\infty}$ состоит из всех тех обших нулей $\lambda_{j}$ системы $\left(\psi_{1}, \ldots, \psi_{n}\right)$, лежаших в $\Gamma$, для которых общая кратность $m_{j}$ меньше кратности $\lambda_{j}$ как нуля $\psi_{i}(z)$. В этом случае $m_{i, j}$ является разностью между кратностью $\lambda_{i, j}$ как нуля $\psi_{i}(z)$ и его кратностью как общего нуля системы $\left(\psi_{1}, \ldots, \psi_{n}\right)$. Предположим, что для каждого $i=1, \ldots, n$ последовательность $\left\{m_{i, j}\right\}_{j=1}^{\infty}$ ограничена и выполнено одно из следующих условий (правильно распределенное множество, удовлетворяюшее этим условиям, называется регулярным.м (см. [1])):

1) сушествует такое число $d_{i}>0$, что кружки радиусов $r_{i, j}=d_{i}\left|\lambda_{i, j}\right|^{1-\rho / 2} \mathrm{c}$ центрами в точках $\lambda_{i, j}$ не пересекаются;

2) существует $d_{i}>0$ такое, что $\left|\lambda_{i, j+1}\right|-\left|\lambda_{i, j}\right|>d_{i}\left|\lambda_{i, j}\right|^{1-\rho}$.

Пусть $C_{i}$ обозначает множество, состоящее из объединения кружков $\left|z-\lambda_{i, j}\right|<$ $r_{i, j}$ в первом случае и $\left|z-\lambda_{i, j}\right|<d_{i}\left|\lambda_{i, j}\right|^{1-\rho}$ во втором. При достаточно малом 
$\delta>0$ в круге $B\left(z_{k}, \delta\left|z_{k}\right|\right)$ функция $\psi_{i, k}(z)$ имеет нули только из числа точек $\left\{\lambda_{i, j}\right\}$, причем с кратностью $m_{i, j}$. Поэтому, повторяя почти дословно рассуждения из $[1$, c. 163-166], нетрудно показать, что оценка, аналогичная (2.11), будет выполнена в круге $B\left(z_{k}, r \delta\left|z_{k}\right|\right)$, но вне множества $C_{i}$. Для регулярности роста системы $\left(\psi_{1}, \ldots, \psi_{n}\right)$ остается потребовать, чтобы было пустым пересечение $C_{1} \cap \cdots \cap C_{n}$ (для некоторых $d_{i}>0, i=1, \ldots, n$ ). Например, если все функции $\psi_{i}(z)$ удовлетворяют условию 2 ), то в случае $\rho=1$ достаточно потребовать существование числа $d>0$ такого, что для любых $i=1, \ldots, n$ и $j=1,2, \ldots$ найдется номер $l=1, \ldots, n$, для которого выполнены неравенства $\left|\lambda_{i, j}-\lambda_{l, s}\right|>d, s=1,2 \ldots$

Рассмотрим некоторые примеры. Поскольку в дальнейшем мы будем иметь дело с функциями экспоненциального типа (т.е. порядка не выше один или конечного типа при порядке один), то примеры приведем для таких функций.

1. Пусть $\psi_{i}(z), i=1, \ldots, n$, являются полиномами. Тогда из сказанного вьше легко следует, что система $\left(\psi_{1}, \ldots, \psi_{n}\right)$ имеет регулярный рост на каждом луче в комплексной плоскости.

2. Пусть $\psi_{i}(z)=\sin [z \pi /(i+1)], i=1, \ldots, n$. Каждая из функций $\psi_{i}(z)$ имеет экспоненциальный тип и регулярньй рост в $\mathbb{C}$ (на каждом луче из $\mathbb{C}$ ). Более того, нулевое множество $\psi_{i}(z)$ является регулярным. Для любых двух не совпадающих нулей $\lambda_{i, j}$ и $\lambda_{l, s}$ функций $\psi_{i}$ и $\psi_{l}$ соответственно выполнена оценка $\left|\lambda_{i, j}-\lambda_{l, s}\right| \geqslant 1$. Таким образом, как показано выше, система $\left(\psi_{1}, \ldots, \psi_{n}\right)$ имеет регулярный рост в $\mathbb{C}$.

\section{3. Пусть}

$$
\psi_{1}(z)=\sin (z \pi), \quad \psi_{2}(z)=\prod_{k=1}^{\infty}\left(1+\frac{z}{k}\right) \prod_{k=1}^{\infty}\left(1-\frac{z}{k+k^{-1}}\right) .
$$

Как и в предыдущем примере, эти функции имеют экспоненциальный тип и регулярные нулевые множества. Однако их не совпадаюшие нули не отделены друг от друга фиксированным расстоянием. Тем не менее, система $\left(\psi_{1}, \psi_{2}\right)$ имеет регулярный рост в $\mathbb{C}$. Покажем это. Регулярность нулевых множеств функций $\psi_{1}$ и $\psi_{2}$ означает, в частности, что (см. [1]) для каждого $\varepsilon>0$ найдется $R>0$ такое, что вне круга $B(0, R)$ и кружков $\left|z-\lambda_{i, j}\right|<1 / 2, j=1,2, \ldots$, выполнено неравенство

$$
\ln \left|\psi_{i}(z)\right| \geqslant h_{\psi_{i}}(z)-\varepsilon|z|, \quad i=1,2,
$$

где $\lambda_{i, j}$ - нули $\psi_{i}(z)$. Поскольку последние вещественны, то отсюда сразу следует регулярность роста системы $\left(\psi_{1}, \psi_{2}\right)$ вдоль каждого луча из $\mathbb{C}$, не лежашего на действительной оси. В отрицательном направлении этой оси регулярность роста $\left(\psi_{1}, \psi_{2}\right)$ следует из регулярности роста каждой из функций по отдельности и совпадения их нулевых множеств в левой полуплоскости. Докажем регулярность роста системы $\left(\psi_{1}, \psi_{2}\right)$ вдоль положительной вещественной полуоси. Пусть $\varepsilon>0$ и $k>R$. Согласно (2.12) имеем

$$
\ln \left|\frac{\psi_{1}(z)}{z-k}\right| \geqslant h_{\psi_{1}}(z)-\varepsilon|z|+\ln 2, \quad|z-k|=\frac{1}{2} .
$$


В силу непрерьвности и однородности функции $h_{\psi_{1}}$ можно считать, что при $k>R$ выполнено неравенство

$$
\left|h_{\psi_{1}}(z)-h_{\psi_{1}}(k)\right| \leqslant \varepsilon|z| \quad \forall z \in B(k, 1 / 2) .
$$

Тогда из предыдушего получаем

$$
\ln \left|\frac{\psi_{1}(z)}{z-k}\right| \geqslant h_{\psi_{1}}(k)-4 \varepsilon k \quad \forall z \in \partial B(k, 1 / 2) .
$$

Поскольку $\psi_{1}(z)(z-k)^{-1}$ не имеет нулей в $B(k, 1 / 2)$, то, последнее неравенство будет выполнено всюду в $B(k, 1 / 2)$. Следовательно, с учетом (2.13) имеем

$$
\ln \left|\frac{\psi_{1}(z)}{z-k}\right| \geqslant h_{\psi_{1}}(z)-9 \varepsilon|z| \quad \forall z \in B(k, 1 / 2) .
$$

Отсюда следует, что

$$
\ln \left|\psi_{1}(z)\right| \geqslant h_{\psi_{1}}(z)-11 \varepsilon|z| \quad \forall z \in B(k, 1 / 2) \backslash B\left(k, e^{-\varepsilon k}\right) .
$$

Вместе с (2.12) это дает нам неравенство

$$
\ln \left|\psi_{1}(z)\right| \geqslant h_{\psi_{1}}(z)-11 \varepsilon|z|, \quad z \notin \bigcup_{k=k_{0}}^{\infty} B\left(k, e^{-\varepsilon k}\right), \quad \operatorname{Re} z>R+1 .
$$

Очевидно, аналогичная оценка с заменой $B\left(k, e^{-\varepsilon k}\right)$ на $B\left(k+1 / k, e^{-\varepsilon k}\right)$ верна и для функции $\psi_{2}(z)$. Требуемая для регулярности роста системы $\left(\psi_{1}, \psi_{2}\right)$ оценка получается теперь из того факта, что для некоторого $k_{0}$ множества $\bigcup_{k=k_{0}}^{\infty} B\left(k, e^{-\varepsilon k}\right)$ и $\bigcup_{k=k_{0}}^{\infty} B\left(k+1 / k, e^{-\varepsilon k}\right)$ не пересекаются.

4. Пусть $\sigma>0$. Положим

$$
\psi_{1}(z)=\sin (z \pi), \quad \psi_{2}(z)=\prod_{k=1}^{\infty}\left(1+\frac{z}{k}\right) \prod_{k=1}^{\infty}\left(1-\frac{z}{k+e^{-k \sigma}}\right) .
$$

Как и в предыдушем примере, система $\left(\psi_{1}, \psi_{2}\right)$ имеет регулярный рост всюду в $\mathbb{C}$, за исключением положительной вешественной полуоси. На этом последнем направлении она не имеет регулярного роста. Действительно, как и в формуле (2.6), при достаточно больших $k$ имеем

$$
\frac{\ln \left|\psi_{1}(z)\right|}{k} \leqslant h_{\psi_{1}}(1)+\frac{\sigma}{2}, \quad|z-k| \leqslant 1 .
$$

Отсюда и из принципа максимума модуля получаем

$$
\ln \left|\frac{\psi_{1}(z)}{z-k}\right| \leqslant h_{\psi_{1}}(k)+\frac{k \sigma}{2}, \quad|z-k| \leqslant 1 .
$$

При тех же $k$ это дает нам неравенство

$$
\ln \left|\psi_{1}\left(k+e^{-k \sigma}\right)\right| \leqslant h_{\psi_{1}}(k)-\frac{k \sigma}{2} .
$$

Таким образом, учитывая неравенство (2.13), мы можем сказать, что необходимая для регулярности роста системы $\left(\psi_{1}, \psi_{2}\right)$ оценка не выполняется в нулевых точках функции $\psi_{2}(z)$ при $\varepsilon<\sigma / 2$. 
ЗАмЕчАНИЕ 2.2. Из последнего примера видно, что в отличие от случая обычной регулярности роста целой функции множество направлений регулярного роста системы функций, вообще говоря, не замкнуто.

Рассмотрим снова целую функцию порядка $\rho$ и конечного типа. Каждая такая функция имеет в некотором смысле "слабый" регулярньй рост всюду в $\mathbb{C}$. Действительно, согласно теореме об оценке снизу целой функции конечного порядка и типа на системе окружностей (см. [1]) для каждого $\delta>0$ найдутся возрастаюшая неограниченная последовательность положительных чисел $\left\{r_{k}\right\}_{k=1}^{\infty}$ и число $A>0$ такие, что $r_{k+1} / r_{k} \leqslant 1+\delta$ и выполнена оценка

$$
\ln |\psi(z)| \geqslant-A|z|^{\rho}, \quad|z|=r_{k}, \quad k=1,2, \ldots
$$

Пусть $y \in \mathbb{S}$. Поскольку $\psi(z)$ имеет конечный тип, то для каждого $\delta_{1} \in(0,1)$ сушествует $B>0$ такое, что при достаточно больших $t>0$

$$
\frac{\ln |\psi(t z)|}{t^{\rho}}<B \quad \forall z \in B\left(y, \delta_{1}\right) .
$$

С учетом двух последних формул, как и в предложении 2.1 , нетрудно показать, что для любых $r>\tau>1$ сушествует $\delta_{0}>0$, удовлетворяюшее условию: для каждого $\delta \in\left(0, \delta_{0}\right)$ найдутся последовательность $\left\{z_{k}\right\}_{k=1}^{\infty} \in \mathbf{E}(y, \delta)$ и число $a>0$ такие, что выполнены неравенства

$$
\ln \left|\frac{\psi(z)}{q_{r \delta}\left(z, z_{k}, \Lambda_{\psi}\right)}\right| \geqslant-a|z|^{\rho}, \quad z \in B\left(z_{k}, \tau \delta\left|z_{k}\right|\right), \quad k \geqslant 1 .
$$

В связи с этим дадим следующее определение.

ОПРЕДЕЛЕнИЕ 2.4. Будем говорить, что система целых функций $\left(\psi_{1}, \ldots, \psi_{n}\right)$ порядка (не вьше) $\rho$ и конечного типа (при порядке $\rho$ ) в $\mathbb{C}$ имеет слабый регулярный рост вдоль луча ty $(y \in \mathbb{S})$, если для любых $r>\tau>1$ сушествует $\delta_{0}>0$, удовлетворяюшее условию: для каждого $\delta \in\left(0, \delta_{0}\right]$ найдутся последовательность $\left\{z_{k}\right\} \in \mathbf{E}(y, \delta)$ и число $A>0$ такие, что

$$
\max _{1 \leqslant i \leqslant n} \ln \left|\frac{\psi_{i}(z)}{q_{r \delta}\left(z, z_{k}, \Lambda\right)}\right| \geqslant-A|z|^{\rho}, \quad z \in B\left(z_{k}, \tau \delta\left|z_{k}\right|\right), \quad k \geqslant 1,
$$

где последовательность $\Lambda=\left\{\lambda_{j}, m_{j}\right\}_{j=1}^{\infty}$-та же, что и в определении 2.3.

ПРЕДЛОЖЕНИЕ 2.3. Пусть $у \in \mathbb{S}$. Предположим, что выполнено следующее. Для любых $r>\tau>1$ существует $\delta_{0}>0$, удовлетворяющее условию: для каждого $\delta \in\left(0, \delta_{0}\right)$ найдутся $a>0$ и последовательности $\left\{z_{k}\right\} \in \mathbf{E}(y, \delta)$, $\left\{r_{k}\right\}$ такие, что $\inf _{k \geqslant 1} r_{k} \geqslant r$ и выполнены неравенства

$$
\max _{1 \leqslant i \leqslant n} \ln \left|\frac{\psi_{i}(z)}{q_{r_{k} \delta}\left(z, z_{k}, \Lambda\right)}\right| \geqslant-a|z|^{\rho}, \quad z \in B\left(z_{k}, \tau \delta\left|z_{k}\right|\right), \quad k \geqslant 1 .
$$

Тогда система $\left(\psi_{1}, \ldots, \psi_{n}\right)$ имеет слабый регулярный рост вдоль луча ty. 
ДоКАЗАТЕЛЬСТВО. Процедура получения оценки в определении 2.4 из неравенства (2.15) такая же, как и в предложении 2.2. Отметим только, что в силу конечности типа функции $\psi_{i}(z)$ число ее нулей в круге $B(0, t)$ оценивается сверху величиной $c t^{\rho}$, где $c>0$ не зависит от $t>0$ (см. [1]). Поэтому не требуется наличие неравенств типа (2.8) и (2.9).

ЗАмЕчАниЕ 2.3. Сравнивая определения 2.3 и 2.4 , легко видеть, что регулярность роста системы $\left(\psi_{1}, \ldots, \psi_{n}\right)$ влечет за собой ее слабую регулярность роста.

ЗАмечАние 2.4. Анализируя доказательство упомянутой вьше теоремы об оценке снизу на окружностях целой функции конечного порядка и типа (см. [1]), легко видеть, что для любых целых функций $\psi_{1}, \ldots, \psi_{n}$ порядка $\rho$ и конечного типа найдется единая система окружностей, на которой для каждой функции $\psi_{i}(z)$ вьполнена оценка типа (2.14). Таким образом (как ранее для регулярного роста), чтобы система $\left(\psi_{1}, \ldots, \psi_{n}\right)$ имела слабый регулярный рост, достаточно наложить некоторые условия на взаимное расположение нулей функций $\psi_{1}, \ldots, \psi_{n}$. В качестве таких условий могут выступать те же условия, что и в случае регулярного роста.

ЗАмЕчАниЕ 2.5. Цитированный во введении результат В.В. Напалкова [2] о разрешимости системы (1.1) для случая, когда $G=\mathbb{C}$ и $f_{1}, \ldots, f_{n}$ не имеют общих нулей, можно сформулировать следуюшим образом. Система уравнений свертки $(1.1)$ разрешима в $H(\mathbb{C})$ при любой допустимой правой части из $H^{n}(\mathbb{C})$ тогда и только тогда, когда система $\left(f_{1}, \ldots, f_{n}\right)$ имеет слабый регулярньй рост всюду в $\mathbb{C}$. Действительно, в рассматриваемом случае функция $q_{\delta}(z, x, \Lambda)$ тождественно равна единице при всех $x$ и $\delta$. Поэтому из неравенства

$$
\left|f_{1}(z)\right|+\cdots+\left|f_{n}(z)\right| \geqslant a \exp [-b|z|] \quad \forall z \in \mathbb{C}
$$

сразу следует слабая регулярность роста системы $\left(f_{1}, \ldots, f_{n}\right)$ в $\mathbb{C}$. Обратно, если система $\left(f_{1}, \ldots, f_{n}\right)$ имеет слабый регулярный рост в $\mathbb{C}$, то для каждого $y \in \mathbb{S}$ найдутся открытый угол $\Gamma_{y}$ (который покрывается совокупностью шаров $\left.B\left(z_{k}, \tau \delta\left|z_{k}\right|\right), \quad k \geqslant 1\right)$ и числа $R_{y}, A_{y}>0$ такие, что $y \in \Gamma_{y}$ и

$$
\max _{1 \leqslant i \leqslant n} \ln \left|f_{i}(z)\right| \geqslant-A_{y}|z| \quad \forall z:|z|>R_{y}
$$

Отсюда, выбирая из системы множеств $\Gamma_{y}, y \in \mathbb{S}$, конечное подпокрытие для окружности $\mathbb{S}$, уже нетрудно получить оценку (2.16).

\section{§3. Достаточные условия разрешимости}

В этом параграфе мы докажем, что, как и в случае одного сверточного уравнения, регулярность роста системы характеристических функций влечет за собой разрешимость соответствуюшей системы уравнений свертки. Предварительно сформулируем и докажем несколько вспомогательных утверждений.

ЛЕмма 3.1. Пусть $\Omega$ - открытое множество в $\mathbb{C}, K$ - замкнутое подмножество $\Omega$ и числа $A>0, a \in(0,1)$ такие, что

$$
\operatorname{dist}(z, \partial \Omega) \geqslant 2 a \exp [-A|z|] \quad \forall z \in K \text {. }
$$


Положим

$$
\Omega^{\prime}=\{z \in \Omega: \operatorname{dist}(z, \partial \Omega) \geqslant a \exp [-A(|z|+1)]\} .
$$

Тогда существуют число с $>0$ и функиия $е(z) \in C^{\infty}(\mathbb{C})$ такие, что

$$
\begin{gathered}
0 \leqslant e(z) \leqslant 1 \quad \forall z \in \mathbb{C}, \quad e(z)=1 \quad \forall z \in K \\
e(z)=0 \quad \forall z \in \mathbb{C} \backslash \Omega^{\prime}, \quad\left|\frac{d e(z)}{d \bar{z}}\right| \leqslant c \exp A|z| \quad \forall z \in \mathbb{C} .
\end{gathered}
$$

ДОКАЗАТЕЛЬСТво. Пусть

$$
\Omega_{m}=K \cap\{z: m-1 \leqslant|z| \leqslant m\}, \quad m=1,2, \ldots
$$

Как обычно (см., например, [13, теорема 1.4.1 и формула (1.4.2)]), мы можем найти функции $e_{m} \in C^{\infty}(\mathbb{C}), m \geqslant 1$, такие, что

$$
\begin{gathered}
0 \leqslant e_{m}(z) \leqslant 1 \quad \forall z \in \mathbb{C}, \quad e_{m}(z)=1, \quad z \in \Omega_{m}, \\
e_{m}(z)=0 \quad \forall z \in \mathbb{C} \backslash\left[\Omega_{m}^{\prime}=\left\{y: \operatorname{dist}\left(y, \Omega_{m}\right) \leqslant a e^{-2 A} e^{-A m}\right\}\right], \\
\left|\frac{d e_{m}(z)}{d \bar{z}}\right| \leqslant \tilde{c} a^{-1} e^{2 A} e^{A m} \quad \forall z \in \mathbb{C},
\end{gathered}
$$

где $\tilde{c}$ не зависит от $a, A$ и $m$.

Пусть $z \in \Omega_{m}^{\prime}$. Тогда $|z|+2 \geqslant m$, и в силу последнего неравенства имеем

$$
\left|\frac{d e_{m}(z)}{d \bar{z}}\right| \leqslant \tilde{c} a^{-1} e^{4 A} e^{A|z|} \quad \forall z \in \mathbb{C} .
$$

Выберем $y \in \Omega_{m}$ такое, что $|y-z| \leqslant a e^{-2 A} e^{-A m}$. Тогда с учетом неравенств $|z|-1 \leqslant m$ и $|z|+1 \geqslant|y|$ получаем

$$
\begin{aligned}
\operatorname{dist}(z, \partial \Omega) & \geqslant \operatorname{dist}(y, \partial \Omega)-|y-z| \geqslant 2 a e^{-A|y|}-a e^{-2 A} e^{-A m} \\
& \geqslant 2 a e^{-A} e^{-A|z|}-a e^{-2 A} e^{A} e^{-A|z|}=a e^{-A} e^{-A|z|}
\end{aligned}
$$

Таким образом, верно включение $\Omega_{m}^{\prime} \subset \Omega^{\prime}, m \geqslant 1$. Определим функцию $e(z)$ по формуле

$$
e(z)=1-\prod_{m=1}^{\infty}\left(1-e_{m}(z)\right) .
$$

Отметим, что в каждой точке $z \in \mathbb{C}$ отлично от единицы не более трех сомножителей этого произведения. С учетом доказанного вьше легко видеть, что $e(z)$ удовлетворяет всем требованиям. Лемма доказана.

Для выпуклой области $D \subset \mathbb{C}$ положим

$$
I_{D}=\left\{z \in \mathbb{C}: H_{D}(z)=+\infty\right\} .
$$

Если $D$ ограничена, то, очевидно, $I_{D}$ - пустое множество. 
Лемма 3.2. Пусть $D$ - выпуклая область в $\mathbb{C}, K$ - выпуклый компакт в $D$ и число $а>0$ такие, что

$$
H_{K}(z)+a|z|<H_{D}(z), \quad z \in \mathbb{C} \backslash\{0\} .
$$

Пусть, кроме того, множество $X \subset \mathbb{S}$ компактно принадлежит int $I_{D}$ (внутренности множества $I_{D}$ ). Тогда для любого $A>0$ найдется выпукльй компакт $L \subset D$ такой, что $K \subset L$ и выполнены неравенства

$$
\begin{gathered}
H_{L}(z)+a|z|<H_{D}(z), \quad z \in \mathbb{C} \backslash\{0\}, \\
H_{L}(z)>A|z| \quad \forall z: z /|z| \in X .
\end{gathered}
$$

ДокАЗАТЕЛЬСтво. Предположим вначале, что $0 \in K$. Тогда

$$
H_{D}(z)>H_{K}(z) \geqslant 0, \quad z \in \mathbb{C} \backslash\{0\} .
$$

Если $I_{D}=\varnothing$, то утверждение тривиально. Если же $I_{D} \neq \varnothing$, то из выпуклости и однородности $H_{D}$ следует, что множество $\mathbb{C} \backslash I_{D}$ является углом с вершиной в начале координат раствора не больше $\pi$. Следовательно, множество $I_{D}$ - угол раствора не меньше $\pi$. Если $I_{D}=\mathbb{C} \backslash\{0\}$, то в качестве $L$ можно взять замкнутый круг с центром в нуле и радиуса $A+1$. Поэтому считаем, что $I_{D} \neq \mathbb{C} \backslash\{0\}$. Пусть числа $\alpha_{1}, \alpha_{2} \in[0,3 \pi]$ такие, что $\alpha_{1} \leqslant \alpha_{2}, \alpha_{2}-\alpha_{1} \leqslant \pi$ и

$$
\operatorname{int} I_{D} \cap \mathbb{S}=\left\{e^{i \alpha}: \alpha \in\left(\alpha_{1}-\frac{\pi}{2}, \alpha_{2}+\frac{\pi}{2}\right)\right\} .
$$

Возьмем произвольное число $\alpha \in\left(\alpha_{1}, \alpha_{2}\right)$ и точку $z \notin I_{D} \cup\{0\}$. Легко видеть, что $\operatorname{Re}\left[e^{-i \alpha} z\right] \leqslant 0$. Отсюда с учетом (3.1) получаем

$$
\operatorname{Re}\left[t e^{-i \alpha} z\right] \leqslant 0<H_{D}(z), \quad t \geqslant 0 .
$$

Если $z \in I_{D}$, то, очевидно, имеем $\operatorname{Re}\left[t e^{-i \alpha} z\right]<H_{D}(z)=+\infty, t \geqslant 0$. Вместе с (3.2) это означает (см. [14]), что $t e^{-i \alpha} \in D$ для всех $\alpha \in\left(\alpha_{1}, \alpha_{2}\right)$ и $t \geqslant 0$.

Поскольку $X$ компактно принадлежит $\operatorname{int} I_{D}$, то для каждого $z \in \bar{X}$ найдется число $\alpha \in\left(\alpha_{1}, \alpha_{2}\right)$ такое, что $\operatorname{Re}\left[e^{-i \alpha} z\right]>0$. Таким образом, для каждого $z \in \bar{X}$ существуют $t(z)>0$ и $\alpha(z) \in\left(\alpha_{1}, \alpha_{2}\right)$ такие, что выполнено неравенство $\operatorname{Re}\left[z t(z) e^{-i \alpha(z)}\right]>A$. Причем по непрерывности это неравенство будет выполнено для одних и тех же $t(z)$ и $\alpha(z)$ в целой окрестности точки $z$. Выбирая из этих окрестностей конечное подпокрытие для $\bar{X}$, найдем точки $y_{k}=t\left(z_{k}\right) e^{-i \alpha\left(z_{k}\right)} \in D$, $k=1, \ldots, m$, такие, что

$$
\max _{1 \leqslant k \leqslant m} \operatorname{Re}\left(y_{k} z\right)>A|z| \quad \forall z: z /|z| \in \bar{X} .
$$

Пусть $L$ - выпуклая оболочка объединения $K \cup \bigcup_{k=1}^{m}\left\{y_{k}\right\}$. Тогда $K \subset L$ и по известной формуле (см. [14])

$$
H_{L}(z)=\max \left\{H_{K}(z), \operatorname{Re}\left(y_{k} z\right), \quad k=1, \ldots, m\right\} .
$$


Отсюда с учетом $(3.1),(3.2)$ и включения $\alpha\left(z_{k}\right) \in\left(\alpha_{1}, \alpha_{2}\right)$ получаем

$$
H_{L}(z)=H_{K}(z)<H_{D}(z)-a|z|, \quad z \notin I_{D} \cup\{0\} .
$$

Кроме того, поскольку $L$ ограничено, то для каждого $z \in I_{D}$ имеем $H_{L}(z)<$ $H_{D}(z)-a|z|=+\infty$. Как и выше, эти неравенства означают, что $L \subset D$. Таким образом, компакт $L$ является искомым для случая $0 \in K$. В общем случае выберем произвольную точку $y_{0} \in K$ и рассмотрим область $D-y_{0}$ и компакт $K-y_{0}$. Для этих множеств, как и выше, построим компакт $L$, удовлетворяюший требуемьм оценкам, где число $A$ заменено на $A+\left|y_{0}\right|$. Тогда компакт $L+y_{0}$ будет, очевидно, искомым. Лемма доказана.

Для дальнейшего нам понадобится специальное семейство векторов. Опишем его. Пусть $\Xi$ обозначает совокупность всех $n$-мерных целочисленных векторов $\left(k_{1}, \ldots, k_{n}\right)$, удовлетворяюших следуюшим условиям:

1) существует номер $j=1, \ldots, n$ такой, что $k_{j}=\cdots=k_{n}=n$;

2) выполнены неравенства $1 \leqslant k_{1}<k_{2}<\cdots<k_{j-1} \leqslant n-1$.

На множестве $\Xi$ введем отношение порядка следуюшим образом. Для произвольных элементов $\left(k_{1}, \ldots, k_{n}\right)$ и $\left(\tilde{k}_{1}, \ldots, \tilde{k}_{n}\right)$ множества $\Xi$ считаем, что $\left(k_{1}, \ldots, k_{n}\right)>\left(\tilde{k}_{1}, \ldots, \tilde{k}_{n}\right)$, если для некоторого номера $i=1, \ldots, n$ выполнены соотношения: $k_{i}>\tilde{k}_{i}$ и $k_{p}=\tilde{k}_{p}, p=1, \ldots, i-1$. Очевидно, что любые два элемента из $\Xi$ сравнимы между собой. Пусть $M-$ число элементов множества $\Xi$. Обозначим через $\left(k_{1}(m), \ldots, k_{n}(m)\right), m=1, \ldots, M$, совокупность элементов $\Xi$, перенумерованных в порядке "возрастания", а через $j(m)=j$ номер из условий $1), 2)$, соответствуюший вектору $\left(k_{1}(m), \ldots, k_{n}(m)\right)$.

Построим теперь специальное покрытие комплексной плоскости, которое понадобится нам при доказательстве основного результата данного параграфа.

Фиксируем произвольное $\varepsilon>0$ и замкнутое подмножество $Z$ единичной окружности $\mathbb{S}$. Пусть $\left(f_{1}, \ldots, f_{n}\right)$ - система целых функций экспоненциального типа в $\mathbb{C}$, удовлетворяющая условиям:

i) $\left(f_{1}, \ldots, f_{n}\right)$ имеет регулярный рост на $Z$ (на каждом луче $t y, y \in Z$ );

ii) $\left(f_{1}, \ldots, f_{n}\right)$ имеет слабый регулярный рост на множестве $\mathbb{S} \backslash Z$.

В силу равномерной непрерьвности индикаторов $h_{f_{i}}(z)$ на компактных подмножествах из $\mathbb{C}$ мы можем выбрать $\delta^{\prime}>0$ такое, что $16(M+1) \delta^{\prime}<1$ и

$$
\sum_{i=1}^{n}\left|h_{f_{i}}(z)-h_{f_{i}}(y)\right|<\varepsilon \quad \forall y \in B\left(z, 10(M+1) \delta^{\prime}\right), \quad \forall z \in \mathbb{S} .
$$

Так как $h_{f_{i}}-$ индикаторная функция для $f_{i}$, то верно неравенство (см. [2])

$$
\ln \left|f_{i}(z)\right| \leqslant h_{f_{i}}(z)+\varepsilon|z|+C^{\prime} \quad \forall z \in \mathbb{C}, \quad i=1, \ldots, n,
$$

где $C^{\prime}>0$ - некоторая постоянная, зависящая от $\varepsilon>0$.

Пусть теперь $y \in Z$. Согласно і) система $\left(f_{1}, \ldots, f_{n}\right)$ имеет регулярный рост вдоль луча $t y$. Для выбранного $\varepsilon>0$ и $r=1 / 2$ через $\delta^{\prime}(y)$ обозначим величину $\delta(r, \varepsilon) / 2(M+1)$, где $\delta(r, \varepsilon)$ - число, сушествование которого требуется в определении 2.3. Для каждого $y \in Z$ фиксируем $\delta(y)>0$ такое, что

$$
\delta(y) \leqslant \min \left\{\delta^{\prime}, \delta^{\prime}(y)\right\} .
$$


Из покрытия $Z$ совокупностью кружков $B(y, \delta(y) / 4), y \in Z$, выделим конечное подпокрытие $B\left(y_{l}, \delta_{l} / 4\right)$, где $\delta_{l}=\delta\left(y_{l}\right), l=1, \ldots, l_{0}$. Через $\left\{z_{k, l}\right\}_{k=1}^{\infty}$ обозначим последовательность $\left\{z_{k}\right\}_{k=k_{0}}^{\infty} \in \mathbf{E}\left(y_{l}\right)$, для которой выполнены неравенства в определении 2.3. Согласно определениям $\mathbf{E}\left(y_{l}\right)$ и $\mathbf{E}\left(y_{l}, \delta\right)$ при помоши изъятия конечного числа элементов из последовательности $\left\{z_{k, l}\right\}$ можно добиться того, чтобы было верно включение $\left\{z_{k, l}\right\} \in \mathbf{E}\left(y_{l}, \delta_{l} / 2\right)$. С учетом этого включения нетрудно видеть, что для каждого $l=1, \ldots, l_{0}$ найдется $R_{l}>0$ такое, что вне круга $B\left(0, R_{l}\right)$ угол (с вершиной в нуле), порожденный кружком $B\left(y_{l}, \delta_{l} / 4\right)$, покрывается объединением $\bigcup_{k} B\left(z_{k, l}, \delta_{l}\left|z_{k, l}\right|\right)$. Пусть

$$
X=\mathbb{S} \backslash\left[\bigcup_{l=1}^{l_{0}} B\left(y_{l}, \frac{\delta_{l}}{4}\right)\right] .
$$

Тогда найдется $\tilde{\delta}>0$ такое, что $8(M+1) \tilde{\delta}<1$, и для каждого $x \in X$ кружок $B(x, 8(M+1) \tilde{\delta})$ компактно принадлежит углу (с вершиной в нуле), порожденному множеством $\mathbb{S} \backslash Z$. Поскольку функции $f_{i}(z), i=1, \ldots, n$, имеют экспоненциальный тип, то для некоторых $A^{\prime}, B^{\prime}>0$ верна оценка

$$
\ln \left|f_{i}(z)\right| \leqslant A^{\prime}|z|+B^{\prime} \quad \forall z \in \mathbb{C}, \quad i=1, \ldots, n .
$$

Согласно условию ii) для $\tau=2(M+1), r=2 \tau$ и каждого $x \in X$ найдем число $\delta_{0}=\delta_{0}(x)$, фигурируюшее в определении 2.4. Для каждого $x \in X$ фиксируем произвольное число $\tilde{\delta}(x)>0$, удовлетворяюшее неравенству

$$
\tilde{\delta}(x) \leqslant \min \left\{\delta^{\prime}, \tilde{\delta}, \delta_{0}(x)\right\} .
$$

Как и выше, найдем точки $x_{l} \in X, l=1, \ldots, l_{1}$, такие, что выполнено включение

$$
X \subset \bigcup_{l=1}^{l_{1}} B\left(x_{l}, \tilde{\delta}_{l} / 2\right),
$$

где $\tilde{\delta}_{l}=\tilde{\delta}\left(x_{l}\right)$. Согласно определению 2.4 для каждого $l=1, \ldots, l_{1}$ мы можем выбрать последовательность $\left\{w_{k, l}\right\} \in \mathbf{E}\left(x_{l}, \tilde{\delta}_{l}\right)$ и число $A_{l}>0$ так, чтобы неравенство в этом определении было выполнено для $\psi_{i}=f_{i}, i=1, \ldots, n$, при $\rho=1$ с заменами $\left\{z_{k}\right\}, A$ и $\delta$ соответственно на $\left\{w_{k, l}\right\}, A_{l}$ и $\tilde{\delta}_{l}$. Как и выше, найдутся $T_{l}>0, l=1, \ldots, l_{1}$, такие, что вне круга $B\left(0, T_{l}\right)$ угол, порожденный кружком $B\left(x_{l}, \tilde{\delta}_{l} / 2\right)$, покрывается объединением $\bigcup_{k=1}^{\infty} B\left(w_{k, l}, 2 \tilde{\delta}_{l}\left|w_{k, l}\right|\right)$.

Пусть $\Theta=\max _{1 \leqslant l \leqslant l_{1}} A_{l}$. Через $U_{k, l}^{i}(d)$ (соответственно $\left.\widetilde{U}_{k, l}^{i}(d)\right)$ обозначим открытое подмножество точек $z$ круга $B\left(z_{k, l}, d \delta_{l}\left|z_{k, l}\right|\right.$ ) (соответственно круга $\left.B\left(w_{k, l}, 2 d \tilde{\delta}_{l}\left|w_{k, l}\right|\right)\right)$, удовлетворяюших неравенству

$$
\ln \left|\frac{f_{i}(z)}{q_{2(M+1) \delta_{l}}\left(z, z_{k, l}, \Lambda\right)}\right|-h_{f_{i}}(z)>-d \varepsilon|z|,
$$

соответственно неравенству

$$
\ln \left|\frac{f_{i}(z)}{q_{4(M+1) \tilde{\delta}_{l}}\left(z, w_{k, l}, \Lambda\right)}\right|>-d \Theta|z|,
$$


где $\Lambda=\left\{\lambda_{j}, m_{j}\right\}$ - общие нули и их кратности функций $f_{1}, \ldots, f_{n}$. В силу выбора величин $\delta_{l}$ (соответственно $\tilde{\delta}_{l}$ и $A_{l}$ ) с учетом определения 2.3 (соответственно определения 2.4) для каждого $d \in(1, M+1]$ множества $\bigcup_{i=1}^{n} U_{k, l}^{i}(d)$ (соответственно $\left.\bigcup_{i=1}^{n} \widetilde{U}_{k, l}^{i}(d)\right)$ и $B\left(z_{k, l}, d \delta_{l}\left|z_{k, l}\right|\right)$ (соответственно $\left.B\left(w_{k, l}, 2 d \tilde{\delta}_{l}\left|w_{k, l}\right|\right)\right)$ совпадают. Пусть $R_{0} \geqslant \max \left\{R_{l}, T_{p}: l=1, \ldots, l_{0} ; p=1, \ldots, l_{1}\right\}$. По построению объединение всех кругов $B\left(z_{k, l}, \delta_{l}\left|z_{k, l}\right|\right)$ и $B\left(w_{k, l}, 2 \tilde{\delta}_{l}\left|w_{k, l}\right|\right)$ содержит множество $\mathbb{C} \backslash B\left(0, R_{0}\right)$. Следовательно, для каждого $d \in(1, M+1]$ любое $z \in \mathbb{C}$, модуль которого не меньше $R_{0}$, лежит в одном (точнее, хотя бы в одном) из множеств $U_{k, l}^{i}(d)$ либо $\widetilde{U}_{k, l}^{i}(d)$. Пусть $x_{0}$ - произвольная точка из $\mathbb{S} \subset B\left(0, R_{0}\right)$ (можно считать, что $R_{0}>1$ ), не совпадаюшая ни с одним из обших нулей $\lambda_{j}$. Очевидно, найдется число $\Theta^{\prime}>0$ такое, что

$$
\max _{1 \leqslant i \leqslant n} \ln \left|\frac{f_{i}(z)}{q_{4(M+1) R_{0}}\left(z, x_{0}, \Lambda\right)}\right|>-\Theta^{\prime} \quad \forall z \in B\left(0,2(M+1) R_{0}\right) .
$$

Для каждого $i=1, \ldots, n$ через $U_{0}^{i}(d)$ обозначим подмножество точек $z$ круга $B\left(0,2 d R_{0}\right)$ таких, что выполнено неравенство

$$
\ln \left|\frac{f_{i}(z)}{q_{4(M+1) R_{0}}\left(z, x_{0}, \Lambda\right)}\right|>-d \Theta^{\prime}
$$

Тогда, как и вьше, для любого $d \in(1, M+1]$ круг $B\left(0,2 d R_{0}\right)$ совпадает с объединением $\bigcup_{i=1}^{n} U_{0}^{i}(d)$. Положим

$$
V_{i}(d)=U_{0}^{i}(d) \cup\left[\bigcup_{k=1}^{\infty} \bigcup_{l=1}^{l_{0}} U_{k, l}^{i}(d)\right] \cup\left[\bigcup_{k=1}^{\infty} \bigcup_{l=1}^{l_{1}} \widetilde{U}_{k, l}^{i}(d)\right] .
$$

Таким образом, для каждого $d \in(1, M+1]$ построено открытое покрытие комплексной плоскости $V_{1}(d), \ldots, V_{n}(d)$. Это покрытие определяется $\varepsilon>0$, функциями $f_{1}, \ldots, f_{n}$, удовлетворяюшими условиям i) и ii), множеством $Z \subset \mathbb{S}$ и числом $M$ элементов множества $\Xi$. Введем еше следуюшее обозначение:

$$
X(d)=\mathbb{S} \cap\left[\bigcup_{l=1}^{l_{1}} B\left(x_{l}, 2 d \tilde{\delta}_{l}\right)\right]
$$

где $\tilde{\delta}_{l}$ и $x_{l}-$ те же, что и выше.

ЛЕмма 3.3. Пусть $1<d<d_{1} \leqslant M+1$. Существуют положительные постояннье $c=c\left(d, d_{1}\right)$ и $\tilde{a}=\tilde{a}\left(d, d_{1}\right)$ такие, что для каждого номера $i=$ $1, \ldots, n$ и любого $z \in V_{i}(d) \backslash\{0\}$ верна оченка

$$
\operatorname{dist}\left(z, \partial V_{i}\left(d_{1}\right)\right) \geqslant \begin{cases}c \exp [-(d+9) \varepsilon|z|], & z /|z| \notin X(d), \\ c \exp [-\tilde{a}|z|], & z /|z| \in X(d) .\end{cases}
$$


ДокАЗАТЕЛЬСТво. Пусть $z /|z| \notin X(d)$. Требуемую оценку достаточно доказать лишь для больших по модулю точек $z$. Поэтому согласно сказанному вьше можно считать, что для некоторых $k=1,2, \ldots$ и $l=1, \ldots, l_{0}$ множество $U_{k, l}^{i}(d)$ содержит $z$ ( $z$ не может принадлежать никакому из множеств $\widetilde{U}_{k, l}^{i}(d)$, поскольку последние лежат в угле, порожденном $X(d))$. Положим

$$
\psi_{k, l}^{i}(y)=\frac{f_{i}(y)}{q_{2(M+1) \delta_{l}}\left(y, z_{k, l}, \Lambda\right)} .
$$

Из оценок (2.2) и (3.4) следует, что

$$
\ln \left|\psi_{k, l}^{i}(y)\right| \leqslant h_{f_{i}}(y)+\varepsilon|y|+C^{\prime} \quad \forall y \in \partial B\left(z_{k, l}, 4(M+1) \delta_{l}\left|z_{k, l}\right|\right) .
$$

Так как $4(M+1) \delta_{l}<1$, то $|y|<2\left|z_{k, l}\right|$ для всех $y \in \partial B\left(z_{k, l}, 4(M+1) \delta_{l}\left|z_{k, l}\right|\right)$. Следовательно, из предыдушего с учетом (3.3) и однородности $h_{f_{i}}$ получаем

$$
\ln \left|\psi_{k, l}^{i}(y)\right| \leqslant h_{f_{i}}\left(z_{k, l}\right)+3 \varepsilon\left|z_{k, l}\right|+C^{\prime} \quad \forall y \in \partial B\left(z_{k, l}, 4(M+1) \delta_{l}\left|z_{k, l}\right|\right) .
$$

По принципу максимума это неравенство будет выполнено и всюду в круге $B\left(z_{k, l}, 4(M+1) \delta_{l}\left|z_{k, l}\right|\right)$. Так как $(M+1) \delta_{l}\left|z_{k, l}\right|>1$ при больших $k$, то, представляя производную $\left(\psi_{k, l}^{i}\right)^{\prime}(y)$ интегралом Коши по единичной окружности с центром в точке $y$, найдем, что для некоторого $C^{\prime \prime}>0$, не зависяшего от $k$ и $l$, имеет место оценка

$$
\ln \left|\left(\psi_{k, l}^{i}\right)^{\prime}(y)\right| \leqslant h_{f_{i}}\left(z_{k, l}\right)+3 \varepsilon\left|z_{k, l}\right|+C^{\prime \prime} \quad \forall y \in B\left(z_{k, l}, 3(M+1) \delta_{l}\left|z_{k, l}\right|\right) .
$$

Поскольку $z \in U_{k, l}^{i}(d) \subset B\left(z_{k, l},(M+1) \delta_{l}\left|z_{k, l}\right|\right)$, то из формулы для первообразной следует, что

$$
\begin{gathered}
\left|\psi_{k, l}^{i}(z)-\psi_{k, l}^{i}(y)\right| \leqslant \exp \left[h_{f_{i}}\left(z_{k, l}\right)+3 \varepsilon\left|z_{k, l}\right|+C^{\prime \prime}\right]|z-y| \\
y \in B\left(z_{k, l}, 3(M+1) \delta_{l}\left|z_{k, l}\right|\right)
\end{gathered}
$$

Пусть $y \in B(z, \exp [-(d+9) \varepsilon|z|])$. Тогда (при большом $|z|) \quad y$ лежит в круге $B\left(z_{k, l}, 3(M+1) \delta_{l}\left|z_{k, l}\right|\right)$, и в силу последнего неравенства будет верна оценка

$$
\left|\psi_{k, l}^{i}(y)\right| \geqslant\left|\psi_{k, l}^{i}(z)\right|-\exp \left[h_{f_{i}}\left(z_{k, l}\right)+3 \varepsilon\left|z_{k, l}\right|-(d+9) \varepsilon|z|+C^{\prime \prime}\right] .
$$

Из соотношения (3.3) и однородности функции $h_{f_{i}}$ следует, что

$$
h_{f_{i}}\left(z_{k, l}\right)<h_{f_{i}}(z)+\varepsilon\left|z_{k, l}\right| .
$$

Вспомним теперь, что в силу выбора величин $\delta_{l}$ имеем $(M+1) \delta_{l}<1 / 2$. Поэтому с учетом включения $z \in B\left(z_{k, l},(M+1) \delta_{l}\left|z_{k, l}\right|\right)$ находим, что

$$
|z| \geqslant\left|z_{k, l}\right|-(M+1) \delta_{l}\left|z_{k, l}\right|>\frac{\left|z_{k, l}\right|}{2} .
$$


Используя последние соотношения и (3.6), получаем

$\left|\psi_{k, l}^{i}(y)\right| \geqslant\left|\psi_{k, l}^{i}(z)\right|-\exp \left[h_{f_{i}}(z)-\varepsilon|z|-d \varepsilon|z|+C^{\prime \prime}\right], \quad y \in B(z, \exp [-(d+9) \varepsilon|z|])$

Так как $z \in U_{k, l}^{i}(d)$, то отсюда с учетом определения $U_{k, l}^{i}(d)$ следует оценка

$\left|\psi_{k, l}^{i}(y)\right| \geqslant \exp \left[h_{f_{i}}(z)-d \varepsilon|z|\right]\left(1-\exp \left[-\varepsilon|z|+C^{\prime \prime}\right]\right), \quad y \in B(z, \exp [-(d+9) \varepsilon|z|])$

Поскольку $d_{1}>d$, то, как и в $(3.3)$, из равномерной непрерывности функции $h_{f_{i}}$ на $\mathbb{S}$ и ее однородности находим, что для любых достаточно больших по модулю точек $z$ и всех $y \in B(z, \exp [-(d+9) \varepsilon|z|])$ выполнено неравенство

$$
h_{f_{i}}(z)-d \varepsilon|z|+\ln \left(1-\exp \left[-\varepsilon|z|+C^{\prime \prime}\right]\right)>h_{f_{i}}(y)-d_{1} \varepsilon|y| .
$$

Следовательно, в силу (3.7) для точек $z$, лежащих вне достаточно большого круга, имеем

$$
\left|\psi_{k, l}^{i}(y)\right| \geqslant \exp \left[h_{f_{i}}(y)-d_{1} \varepsilon|y|\right] \quad \forall y \in B(z, \exp [-(d+9) \varepsilon|z|]) .
$$

Кроме того, при больших значениях $|z|$ неравенство $d_{1}>d$ и то, что $z \in$ $B\left(z_{k, l}, d \delta_{l}\left|z_{k, l}\right|\right)$, влекут за собой включение $y \in B\left(z_{k, l}, d_{1} \delta_{l}\left|z_{k, l}\right|\right)$ для всех $y \in B(z, \exp [-(d+9) \varepsilon|z|])$. Таким образом, согласно определению множества $U_{k, l}^{i}\left(d_{1}\right)$ круг $B(z, \exp [-(d+9) \varepsilon|z|])$ лежит в нем, а следовательно, и в $V_{i}\left(d_{1}\right)$. Это дает нам первое из требуемых в лемме неравенств. Второе неравенство доказывается аналогично с использованием (3.5). Лемма доказана.

Лемма 3.4. Для каждого $d \in(1, M+1]$ существует разбиение единиць $E_{i} \in C^{\infty}(\mathbb{C}), i=1, \ldots, n$, соответствующее покрытию $V_{1}(d), \ldots, V_{n}(d)$ комплексной плоскости (т.е. $E_{i}$ равна нулю в окрестности границы множества $V_{i}(d)$ и вне его), такое, что для некоторых $\tilde{c}, \tilde{b}>0$ выполнены неравенства

$$
\left|\frac{d E_{i}(z)}{d \bar{z}}\right| \leqslant \begin{cases}\tilde{c} \exp [(d+9) \varepsilon|z|], & z /|z| \notin X(d), \\ \tilde{c} \exp [\tilde{b}|z|], & z /|z| \in X(d), \quad i=1, \ldots, n .\end{cases}
$$

ДокАЗАТЕЛЬСТво. Пусть $d_{1} \in(1, d)$. Введем множества

$$
W_{i}=\left\{z \in V_{i}\left(d_{1}\right) \backslash\{0\}: z /|z| \notin X\left(d_{1}\right)\right\}, \quad \widetilde{W}_{i}=V_{i}\left(d_{1}\right) \backslash W_{i}, \quad i=1, \ldots, n .
$$

Кроме того, для каждого $i=1, \ldots, n$ положим

$$
\Omega_{i}=\bigcup_{z \in W_{i}} B\left(z, c \exp \left[-\left(d_{1}+9\right) \varepsilon|z|\right]\right), \quad \widetilde{\Omega}_{i}=\bigcup_{z \in \widetilde{W}_{i}} B(z, c \exp [-\tilde{a}|z|]),
$$

где $c=c\left(d_{1}, d\right)$ и $\tilde{a}=\tilde{a}\left(d_{1}, d\right)$ - положительные постоянные, сушествование которых утверждается в лемме 3.3. Для каждого $i=1, \ldots, n$ через $e_{i}(z)$ обозначим функцию, построенную в лемме 3.1 для множеств $\Omega=\Omega_{i}, K=\bar{W}_{i}$ и чисел 
$A=\left(d_{1}+9\right) \varepsilon, a=c / 2$ (очевидно, можно считать, что $\left.c<2\right)$. Аналогичные функции $\tilde{e}_{i}(z)$ найдем также для множеств $\Omega=\widetilde{\Omega}_{i}, K=\overline{\widetilde{W}}_{i}$ и чисел $A=\tilde{a}, a=c / 2$. Положим теперь

$$
E_{i}(z)=\left(e_{i}(z)+\tilde{e}_{i}(z)\right)\left[\sum_{j=1}^{n}\left(e_{j}(z)+\tilde{e}_{j}(z)\right)\right]^{-1}, \quad i=1, \ldots, n .
$$

Согласно лемме $3.1 e_{i}(z)$ и $\tilde{e}_{i}(z)$ неотрищательны. Поэтому $E_{i}(z) \geqslant 0 \forall z$. По построению $E_{i}(z) \leqslant 1 \forall z$ и $\sum E_{i}(z) \equiv 1$. По лемме $3.1 e_{i}(z) \quad\left(\tilde{e}_{i}(z)\right)$ равна нулю в окрестности границы множества $\Omega_{i}\left(\widetilde{\Omega}_{i}\right)$ и вне его. По лемме 3.3 множества $\Omega_{i}$ и $\widetilde{\Omega}_{i}$ лежат в $V_{i}(d)$. Следовательно, $E_{i}(z)$ равна нулю в окрестности гранищы множества $V_{i}(d)$ и вне его. Таким образом, $E_{i}(z)$ являются разбиением единицы, соответствуюшим покрытию $V_{i}(d), i=1, \ldots, n$.

По лемме 3.1 функции $e_{i}(z)$ и $\tilde{e}_{i}(z)$ ограничены сверху единицей всюду в $\mathbb{C}$ и равны единице соответственно на $W_{i}$ и $\widetilde{W}_{i}$. Кроме того, множества $V_{i}\left(d_{1}\right)=W_{i} \cup \widetilde{W}_{i}$, $i=1, \ldots, n$, покрьвают в совокупности всю комплексную плоскость. Следовательно, имеют место неравенства

$$
1 \leqslant \sum_{i=1}^{n}\left(e_{i}(z)+\tilde{e}_{i}(z)\right) \leqslant 2 n \quad \forall z
$$

Но тогда

$$
\left|\frac{d E_{i}(z)}{d \bar{z}}\right| \leqslant 2 n \sum_{j=1}^{n}\left(\left|\frac{d e_{i}(z)}{d \bar{z}}\right|+\left|\frac{d \tilde{e}_{i}(z)}{d \bar{z}}\right|\right) .
$$

Отсюда с учетом оценок на производные функций $e_{i}(z)$ и $\tilde{e}_{i}(z)$ в лемме 3.1 получаем второе неравенство настоящей леммы. Пусть $z$ таково, что $z /|z| \notin X(d)$. Тогда $\tilde{e}_{i}(z)=0$ при достаточно большом значении $|z|$. Действительно, согласно лемме $3.1 \tilde{e}_{i}(z)=0 \forall z \notin \widetilde{\Omega}_{i}$, а поскольку $d>d_{1}$, то из определения $\widetilde{\Omega}_{i}$ следует, что для некоторого $R>0$ множество $\widetilde{\Omega}_{i} \backslash B(0, R)$ лежит в угле, порожденном $X(d)$. Таким образом, для указанных $z$ имеем

$$
\left|\frac{d E_{i}(z)}{d \bar{z}}\right| \leqslant 2 n \sum\left|\frac{d e_{j}(z)}{d \bar{z}}\right|
$$

Отсюда, как и выше, получаем первое неравенство нашей леммы.

Для последовательности $\Lambda=\left\{\lambda_{j}, m_{j}\right\}$ общих нулей и их кратностей функций $f_{1}, \ldots, f_{n}$ экспоненциального типа определим функцию $f(z)$ по формуле

$$
f(z)=z^{m_{0}} \prod_{j=1, \lambda_{j} \neq 0}^{\infty}\left(1-\frac{z}{\lambda_{j}}\right)^{m_{j}} \exp \left(\frac{m_{j} z}{\lambda_{j}}\right), \quad z \in \mathbb{C},
$$

где $m_{0}-$ кратность общего нуля $z=0$ функций $f_{1}, \ldots, f_{n}$ (возможно, равная нулю). Поскольку точки $\lambda_{j}, j=1,2, \ldots$, с учетом их кратностей являются нулями (частью нулей) целой функции экспоненциального типа, скажем $f_{1}$, то (см. $[15$, с. 25]) 
наше произведение сходится во всей плоскости и представляет собой целую функцию. Кроме того, $f(z)$ обрашается в нуль только в точках $\lambda_{j}$, причем с кратностью $m_{j}$. Для всех $k=1,2, \ldots$ и $l=1, \ldots, l_{0}$ положим

$$
\psi_{k, l}(z)=\left\{\begin{array}{r}
\ln |f(z)|, \quad z \notin B\left(z_{k, l}, 4(M+1) \delta_{l}\left|z_{k, l}\right|\right), \\
\max \left\{\ln |f(z)| ; \ln \left|\frac{f(z)}{q_{2(M+1) \delta_{l}}\left(z, z_{k, l}, \Lambda\right)}\right|\right\} \\
z \in B\left(z_{k, l}, 4(M+1) \delta_{l}\left|z_{k, l}\right|\right) .
\end{array}\right.
$$

Для всех $k=1,2, \ldots$ и $l=1, \ldots, l_{1}$ определим еще функцию $\tilde{\psi}_{k, l}(z)$ по предыдущей формуле, где $B\left(z_{k, l}, 4(M+1) \delta_{l}\left|z_{k, l}\right|\right)$ и $q_{2(M+1) \delta_{l}}\left(z, z_{k, l}, \Lambda\right)$ нужно заменить соответственно на $B\left(w_{k, l}, 8(M+1) \tilde{\delta}_{l}\left|w_{k, l}\right|\right)$ и $q_{4(M+1) \tilde{\delta}_{l}}\left(z, w_{k, l}, \Lambda\right)$. Пусть также

$$
\psi_{0}(z)=\left\{\begin{array}{c}
\ln |f(z)|, \quad z \notin B\left(x_{0}, 8(M+1) R_{0}\right), \\
\max \left\{\ln |f(z)| ; \ln \left|\frac{f(z)}{q_{4(M+1) R_{0}}\left(z, x_{0}, \Lambda\right)}\right|\right\}, \\
z \in B\left(x_{0}, 8(M+1) R_{0}\right) .
\end{array}\right.
$$

Функции $\psi_{k, l}, \tilde{\psi}_{k, l}, \psi_{0}$ субгармоничны в $\mathbb{C}$. Действительно, при наличии у них полунепрерывности сверху их субгармоничность следует из очевидного вьполнения интегрального неравенства для субгармонических функций в каждой точке из $\mathbb{C}$ (поскольку это неравенство выполнено для субгармонических функций, через которые определяются $\left.\psi_{k, l}, \tilde{\psi}_{k, l}, \psi_{0}\right)$. Полунепрерывность же функций $\psi_{k, l}, \tilde{\psi}_{k, l}, \psi_{0}$ требует проверки лиш соответственно на границах кругов $B\left(z_{k, l}, 4(M+1) \delta_{l}\left|z_{k, l}\right|\right), B\left(w_{k, l}, 8(M+1) \tilde{\delta}_{l}\left|w_{k, l}\right|\right)$ и $B\left(x_{0}, 8(M+1) R_{0}\right)$.

Необходимая нам полунепрерьвность сверху легко вытекает из неравенства

$$
\ln |f(z)| \geqslant \ln \left|\frac{f(z)}{q_{\delta}(z, x, \Lambda)}\right|
$$

выполненного согласно $(2.2)$ для всех $x \neq \lambda_{j}, j=1,2, \ldots$, и $\delta>0$ на границе круга $B(x, 2 \delta|x|)$. Положим теперь

$$
\psi(z)=\sup \left\{\psi_{0}(z), \psi_{k, l}(z), \tilde{\psi}_{p, r}(z)\right\}, \quad z \in \mathbb{C},
$$

где супремум берется по всевозможным значениям индексов $k, l, p, r$. Функция $\psi(z)$ субгармонична в $\mathbb{C}$, поскольку на самом деле в окрестности каждой точки $z \in \mathbb{C}$ представляет собой максимум лишь конечного числа субгармонических функций. Остальные функции там совпадают, так как некоторая окрестность каждой точки из $\mathbb{C}$ пересекается лишш с конечным числом кругов $B\left(z_{k, l}, 4(M+1) \delta_{l}\left|z_{k, l}\right|\right)$ и $B\left(w_{k, l}, 8(M+1) \tilde{\delta}_{l}\left|w_{k, l}\right|\right)$.

По построению в каждой точке $z \in B\left(x_{0}, 8(M+1) R_{0}\right)$ выполнено неравенство

$$
\psi(z) \geqslant \psi_{0}(z) \geqslant \ln \left|\frac{f(z)}{q_{4(M+1) R_{0}}\left(z, x_{0}, \Lambda\right)}\right| .
$$


Согласно определениям функций $f(z)$ и $q_{4(M+1) R_{0}}\left(z, x_{0}, \Lambda\right)$ их частное не имеет нулей в $B\left(x_{0}, 4(M+1) R_{0}\right)$. Поскольку круг $B\left(0, R_{0}\right)$ компактно принадлежит $B\left(x_{0}, 4(M+1) R_{0}\right)$, то в силу последнего неравенства функция $\psi(z)$ ограничена снизу на $B\left(0, R_{0}\right)$.

Для каждого $i=1, \ldots, n$ положим

$$
F_{i}(z)=\frac{f_{i}(z)}{f(z)}, \quad z \in \mathbb{C} .
$$

Из определения $f(z)$ и множества $V_{i}(M+1)$ следует, что функция $F_{i}(z)$ аналитическая и не имеет нулей на замыкании множества $V_{i}(M+1)$.

Лемма 3.5. Существует $C>0$ такое, ито для каждого $i=1, \ldots, n u$ каждого $d \in(1, M+1]$ выполнено неравенство

$$
\ln \left|F_{i}(z)\right|+\psi(z) \geqslant \begin{cases}H_{K_{i}}(z)-d \varepsilon|z|, & z \in V_{i}(d), \quad z /|z| \notin X(d), \\ -d \Theta|z|, & z \in V_{i}(d), \quad z /|z| \in X(d) .\end{cases}
$$

Здесь $\Theta-$ то же, что и при определении множеств $\widetilde{U}_{k, l}^{i}(d)$.

ДоказАТельСТво. Пусть $z \in V_{i}(d) \backslash B\left(0, R_{0}\right)$ ( $R_{0}$ определено вьше) такое, что $z /|z| \notin X(d)$. Тогда, как уже отмечалось, для некоторых $k, l$ точка $z$ принадлежит $U_{k, l}^{i}(d)$. В силу определений $\psi(x)$ и $U_{k, l}^{i}(d)$ получаем

$$
\begin{aligned}
\ln \left|F_{i}(z)\right|+\psi(z) & \geqslant \ln \left|F_{i}(z)\right|+\ln \left|\frac{f(z)}{q_{2(M+1) \delta_{l}}\left(z, z_{k, l}, \Lambda\right)}\right| \\
& =\ln \left|\frac{f_{i}(z)}{q_{2(M+1) \delta_{l}}\left(z, z_{k, l}, \Lambda\right)}\right| \geqslant h_{f_{i}}(z)-d \varepsilon|z| .
\end{aligned}
$$

Поскольку $K_{i}-$ сопряженная диаграмма $f_{i}$, то по теореме Полиа (см. [1]) верно равенство $H_{K_{i}}(x)=h_{f_{i}}(x), x \in \mathbb{C}$. Вместе с предыдушим это доказывает лемму в рассматриваемом случае. Аналогично доказывается требуемое неравенство и для точек $z \in V_{i}(d) \backslash B\left(0, R_{0}\right)$ таких, что $z /|z| \in X(d)$. Что касается точек $z \in$ $V_{i}(d) \cap B\left(0, R_{0}\right)$, необходимая оценка следует здесь из замечаний, сделанных ранее относительно ограниченности $\psi$ и аналитичности $F_{i}$.

Лемма 3.6. Существует $C>0$ такое, что для каждого $i=1, \ldots, n$ выполнено неравенство

$$
\ln \left|F_{i}(z)\right|+\psi(z) \leqslant C+H_{K_{i}}(z)+8 \varepsilon|z|, \quad z \in \mathbb{C} .
$$

ДокАЗАТЕльСТво. Достаточно показать, что требуемое неравенство верно, если $\psi$ заменить на какую-либо функцию из семейства $\left\{\psi_{0}, \psi_{k, l}, \tilde{\psi}_{p, r}\right\}$. Пусть $\psi=\psi_{0}$. Очевидно, что необходимая оценка требует доказательства лишш в точ$\operatorname{kax} z$ с достаточно большим модулем. Для таких $z$ имеет место равенство $\psi_{0}(z)=$ $\ln |f(z)|$. Поэтому с учетом тождества $H_{K_{i}} \equiv h_{f_{i}}$ и (3.4) получаем

$$
\ln \left|F_{i}(z)\right|+\psi_{0}(z)=\ln \left|f_{i}(z)\right| \leqslant H_{K_{i}}(z)+\varepsilon|z|+C^{\prime} .
$$


Пусть теперь $\psi=\tilde{\psi}_{p, r}$. Случай, когда $\tilde{\psi}_{p, r}(z)=\ln |f(z)|$, уже рассмотрен. Поэтому можно считать, что $z \in B\left(w_{p, r}, 8(M+1) \tilde{\delta}_{r}\left|w_{p, r}\right|\right)$ и

$$
\tilde{\psi}_{p, r}(z)=\ln \left|\frac{f(z)}{q_{4(M+1) \tilde{\delta}_{r}}\left(z, w_{p, r}, \Lambda\right)}\right| .
$$

Как и в лемме 3.3, из $(2.2),(3.3),(3.4)$ и принципа максимума следует, что

$$
\ln \left|F_{i}(z)\right|+\tilde{\psi}_{p, r}(z) \leqslant h_{f_{i}}\left(w_{p, r}\right)+3 \varepsilon\left|w_{p, r}\right|+C^{\prime} .
$$

Так как $z \in B\left(w_{p, r}, 8(M+1) \tilde{\delta}_{r}\left|w_{p, r}\right|\right)$ и $16(M+1) \tilde{\delta}_{r}<1$, то $\left|w_{p, r}\right|<2|z|$. Применяя еше раз (3.3), находим, что $h_{f_{i}}\left(w_{p, r}\right) \leqslant h_{f_{i}}(z)+\varepsilon\left|w_{p, r}\right|$. Следовательно,

$$
\ln \left|F_{i}(z)\right|+\tilde{\psi}_{p, r}(z) \leqslant h_{f_{i}}(z)+8 \varepsilon|z|+C^{\prime}
$$

С учетом тождества $H_{K_{i}} \equiv h_{f_{i}}$ получаем требуемое неравенство. Аналогично рассматривается и случай $\psi=\psi_{k, l}$. Лемма доказана.

Пусть $\alpha(s)=\left(\alpha_{1}, \ldots, \alpha_{s}\right)$ обозначает $s$-мерный целочисленньй вектор такой, что $1 \leqslant \alpha_{p} \leqslant n$ при всех $p=1, \ldots, s$. Через $V_{\alpha(s)}(d)$ обозначим пересечение $V_{\alpha_{1}}(d) \cap \cdots \cap V_{\alpha_{s}}(d)$, где $V_{p}(d)$ - элемент описанного вьше покрытия комплексной плоскости. При этом в случае $s=1$, конечно, естественнее использовать обозначение $V_{p}(d)$ вместо $V_{\alpha(s)}(d)$. Пусть $\left(k_{1}(m), \ldots, k_{n}(m)\right), 1 \leqslant m \leqslant M,-$ элемент введенного ранее множества $\Xi$ и $j(m)$ - определенный при описании $\Xi$ номер, который соответствует данному элементу. Введем теперь пространство, которое мы обозначим через $\Phi_{m}$. Оно состоит из всех наборов функций $\left\{\varphi_{\alpha(s)}^{i}\right\}$ (где $s$ пробегает множество $\{1, \ldots, j(m)\}, \alpha(s)$ - всевозможные указанные выше $s$-мерные векторы, а $i$ меняется в пределах от $k_{s-1}(m)$ до $n$ при $s>1$ и в пределах от 1 до $n$ при $s=1)$, удовлетворяющих следующим условиям:

1) для всех возможных значений индексов $i$ и $\alpha(s)$ функция $\varphi_{\alpha(s)}^{i}$ аналитична на множестве $V_{\alpha(s)}(M-m+2)$;

$2)$ если $V_{\alpha(s)}(M-m+2)=\varnothing$, то $\varphi_{\alpha(s)}^{i}$ тождественно равна нулю при всех возможных $i$;

3) для всех возможных $s$ и $i$ набор $\left\{\varphi_{\alpha(s)}^{i}\right\}$ является коцепю, т.е. $\varphi_{\alpha(s)}^{i}-$ кососимметрическая функция индексов $\alpha_{1}, \ldots, \alpha_{s}$, составляюших $\alpha(s)$.

Значком $\Delta$ обозначим обычный кограничный оператор, ставяший в соответствие коцепи $\varphi_{\alpha(s)}$ коцепь $\varphi_{\alpha(s+1)}$ по известному правилу. В дальнейшем равенство $\varphi_{\alpha(s+1)}=\Delta \varphi_{\alpha(s)}$ означает совпадение функции $\varphi_{\alpha(s+1)}$ и элемента коцепи $\Delta \varphi_{\alpha(s)}$, который соответствует индексу $\alpha(s+1)$.

Мы все подготовили для того, чтобы перейти к формулировке и доказательству основного результата данного параграфа.

Теорема 3.7. Пусть области $D, G, G_{i}$, компакты $K_{i}$, операторы $\mathbf{M}_{i} u$ функции $f_{i}-$ те же, что и во введении. Предположим, что выполнень следующие условия:

1) система $\left(f_{1}, \ldots, f_{n}\right)$ имеет регулярный рост на луче $t z, t>0$, для каждого z из замыкания множества $\mathbb{S} \backslash I_{G}$; 
2) система $\left(f_{1}, \ldots, f_{n}\right)$ имеет слабый регулярный рост на луче $t z, t>0$, для кажсдого $z \in \mathbb{S} \cap I_{G}$.

Тогда система уравнений свертки $\mathbf{M}_{1}[h]=g_{1}, \ldots, \mathbf{M}_{n}[h]=g_{n}$ разрешима в пространстве $H(D)$ при любой допустимой правой части $\left(g_{1}, \ldots, g_{n}\right) \in$ $H\left(G_{1}\right) \times \cdots \times H\left(G_{n}\right)$.

ДокАЗАтЕльство. Согласно лемме 1.2 достаточно показать, что для любой функции $F \in I(D, \Lambda)$ найдется элемент $\left(\varphi_{1}, \ldots, \varphi_{n}\right)$ пространства $P_{G_{1}} \times \cdots \times P_{G_{n}}$ такой, что в комплексной плоскости вьполнено тождество

$$
F(z) \equiv \varphi_{1}(z) f_{1}(z)+\cdots+\varphi_{n}(z) f_{n}(z)
$$

Прежде всего мы хотим установить некоторые вспомогательные неравенства. Поскольку $F$ лежит в $I(D, \Lambda)$, а следовательно, и в $P_{D}$, то в силу определения последнего найдутся компакт $K \subset D$ и число $\tilde{A}>0$ такие, что

$$
|F(z)| \leqslant \tilde{A} \exp \left[H_{K}(z)\right] \quad \forall z \in \mathbb{C}
$$

Область $D$ представляет из себя сумму множеств $G+K_{1}+\cdots+K_{n}$. Поэтому легко видеть, что найдется выпуклый компакт $K_{0} \subset G$, для которого верно включение $K \subset K_{0}+K_{1}+\cdots+K_{n}$. Из этого включения следует, что всюду в $\mathbb{C}$ опорная функция компакта $K$ не превосходит опорной функции суммы $K_{0}+K_{1}+\cdots+K_{n}$. Учитывая теперь, что опорная функция суммы выпуклых множеств равна сумме опорных функций этих множеств, получаем неравенство

$$
|F(z)| \leqslant \tilde{A} \exp \left[H_{K_{0}}(z)+H_{K_{1}}(z)+\cdots+H_{K_{n}}(z)\right] \quad \forall z \in \mathbb{C} .
$$

Поскольку некоторое вздутие компакта $K_{0}$ все еще будет лежать в области $G$, то найдется $\varepsilon>0$ такое, что

$$
H_{K_{0}}(z)+(n M(M+11)+10) \varepsilon|z|<H_{G}(z) \quad \forall z \neq 0
$$

где $M$ - число элементов множества $\Xi$. В силу равномерной непрерывности функ-

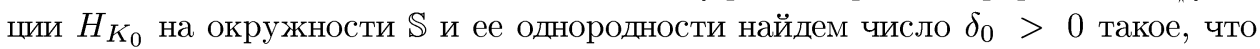
$10 M \delta_{0}<1$ и имеет место неравенство

$$
\left|H_{K_{0}}(z)-H_{K_{0}}(y)\right|<\varepsilon \quad \forall y \in B\left(z, 10 M \delta_{0}\right), \quad z \in \mathbb{S} .
$$

Пусть $Z$ обозначает замыкание множества $\mathbb{S} \backslash I_{G}$. Для выбранного числа $\varepsilon>0$ и множества $Z$ рассмотрим построенное выше покрытие комплексной плоскости $V_{1}(d), \ldots, V_{n}(d)$. Мы можем это сделать, поскольку выполнение условий і) и іi) для функций $f_{1}, \ldots, f_{n}$ предполагается в нашей теореме. При этом можно считать, что определенные при построении этого покрытия числа $\delta_{l}$ и $\tilde{\delta}_{p}$ удовлетворяют неравенству $\delta_{l}, \tilde{\delta}_{p}<\delta_{0}, l=1, \ldots, l_{0}, p=1, \ldots, l_{1}$.

Дальнейшее доказательство теоремы представляет из себя цепочку последовательных индуктивных построений, последнее звено которой обеспечит существование функций $\varphi_{1}, \ldots, \varphi_{n}$ с нужными свойствами. Другими словами, мы хотим показать, что для каждого $m=1, \ldots, M$ существует набор функщий $\left\{\varphi_{\alpha(s), m}^{i}\right\} \in \Phi_{m}$, 
удовлетворяюший перечисленным ниже условиям. Сушествование такого набора для $m=M$ повлечет за собой существование требуемых функций $\varphi_{1}, \ldots, \varphi_{n}$.

Прежде чем сформулировать указанные условия, введем еще некоторые обозначения. Чтобы сделать формулировки условий и дальнейшие рассуждения более компактными, дополнительно к набору $\left\{\varphi_{\alpha(s), m}^{i}\right\}$, где индексы меняются в пределах, указанных выше, введем функции $\varphi_{\alpha(1), m}^{0}$, или, что то же самое, функции $\varphi_{j, m}^{0}$, которые для всех $j=1, \ldots, n$ и $m=1, \ldots, M$ равны $F / f$ ( $f$ построена выше по функциям $\left.f_{1}, \ldots, f_{n}\right)$. Отметим, что функция $F / f$ является целой, поскольку $F \in I(D, \Lambda)$, т.е. $F$ обращается в нуль по крайней мере в точках $\lambda_{j}$ с кратностью, не меньшей чем $m_{j}$, где $\Lambda=\left\{\lambda_{j}, m_{j}\right\}$ - все нули и их кратности функции $f$. Кроме того, мы здесь и далее считаем, что $k_{0}(m)=0$ для всех $m=1, \ldots, M$. Итак, для каждого $m=1, \ldots, M$ будем строить набор $\left\{\varphi_{\alpha(s), m}^{i}\right\} \in \Phi_{m}$, удовлетворяюший следующим условиям.

1) Пусть $j(m)$ - число, определенное при описании целочисленных векторов $k(m)=\left(k_{1}(m), \ldots, k_{n}(m)\right)$. Для каждого $s=1, \ldots, j(m)$ и каждого вектора $\alpha(s)$ вьполнено равенство

$$
\varphi_{\alpha(s), m}^{k_{s-1}(m)}(z)=\sum_{i=k_{s-1}(m)+1}^{n} \varphi_{\alpha(s), m}^{i}(z) F_{i}(z), \quad z \in V_{\alpha(s)}(M-m+2)
$$

2 ) Для каждого $s=1, \ldots, j(m)$ и каждого $i=k_{s-1}(m)+1, \ldots, n$ сушествуют аналитические коцепи $h_{\alpha(s), m}^{i, j}, j=1, \ldots, 4$, такие, что

$$
\varphi_{\alpha(s), m}^{i}(z)=\sum_{j=1}^{4} h_{\alpha(s), m}^{i, j}(z), \quad z \in V_{\alpha(s)}(M-m+2)
$$

и выполнено следуюшее:

а) если среди компонент вектора $\alpha(s)$ нет компоненты, равной $i$, то $h_{\alpha(s), m}^{i, 1} \equiv 0$;

б) $h_{\alpha(s), m}^{k_{s-1}(m)+1,2} \equiv 0$ для всех $s=1, \ldots, j(m)$;

если $k_{s}(m)=k_{s-1}(m)+1$, то

$$
h_{\alpha(s), m}^{i, 2} \equiv 0 \quad \forall i=k_{s-1}(m)+2, \ldots, n
$$

если $k_{s}(m)>k_{s-1}(m)+1$, то при $i=k_{s-1}(m)+2, \ldots, k_{s}(m)-1$

$$
h_{\alpha(s), m}^{i, 2}(z)=\sum_{r=k_{s-1}(m)+1}^{i-1} \varphi_{\alpha(s), m}^{i, r}(z) F_{r}(z), \quad z \in V_{\alpha(s)}(M-m+2)
$$

а при $i=k_{s}(m), \ldots, n$

$$
h_{\alpha(s), m}^{i, 2}(z)=\sum_{r=k_{s-1}(m)+1}^{k_{s}(m)-1} \varphi_{\alpha(s), m}^{i, r}(z) F_{r}(z), \quad z \in V_{\alpha(s)}(M-m+2)
$$


здесь $\varphi_{\alpha(s), m}^{i, r}$ - аналитические коцепи такие, что

$$
\Delta \varphi_{\alpha(s), m}^{i, r}(z)=\varphi_{\alpha(s+1), l(m, s, r)}^{i}(z), \quad z \in V_{\alpha(s+1)}(M-m+2)
$$

и номер $l(m, s, r)$ однозначно определяется равенствами: $j(l(m, s, r))=s+1$, $k_{1}(l(m, s, r))=k_{1}(m), \ldots, k_{s-1}(l(m, s, r))=k_{s-1}(m), k_{s}(l(m, s, r))=r ;$

в) если $k_{s}(m)=k_{s-1}(m)+1$, то

$$
h_{\alpha(s), m}^{i, 3} \equiv 0 \quad \forall i=k_{s-1}(m)+1, \ldots, n
$$

если $k_{s}(m)>k_{s-1}(m)+1$, то

$$
\begin{gathered}
h_{\alpha(s), m}^{i, 3}(z)=-\sum_{j=i+1}^{n} \varphi_{\alpha(s), m}^{j, i}(z) F_{j}(z), \quad z \in V_{\alpha(s)}(M-m+2), \\
i=k_{s-1}(m)+1, \ldots, k_{s}(m)-1, \quad h_{\alpha(s), m}^{i, 3} \equiv 0, \quad i=k_{s}(m), \ldots, n ;
\end{gathered}
$$

г) если $k_{s-1}(m)=s-1\left(\right.$ т.е. $\left.k_{j}(m)=k_{j-1}(m)+1 \forall j=1, \ldots, s-1\right)$, то

$$
h_{\alpha(s), m}^{i, 4} \equiv 0 \quad \forall i=k_{s-1}(m)+1, \ldots, n
$$

в противном случае

$$
\begin{gathered}
h_{\alpha(s), m}^{i, 4}(z)=\sum_{p} \sum_{r=k_{p-1}(m)+1}^{k_{p}(m)-1}(-1)^{s-p} \varphi_{\alpha(s), t(m, s, p, r)}^{i, k_{s-1}(m)}(z) F_{r}(z), \\
z \in V_{\alpha(s)}(M-m+2), \quad i=k_{s-1}(m)+1, \ldots, n
\end{gathered}
$$

где внешнее суммирование ведется по всем $p=1, \ldots, s-1$ таким, что $k_{p}(m)>$ $k_{p-1}(m)+1$, и номер $t(m, s, p, r)$ однозначно определяется из соотношений:

$$
\begin{gathered}
j(t(m, s, p, r))=s, \\
k_{1}(t(m, s, p, r))=k_{1}(m), \ldots, k_{p-1}(t(m, s, p, r))=k_{p-1}(m), \\
k_{p}(t(m, s, p, r))=r \\
k_{p+1}(t(m, s, p, r))=k_{p}(m), \ldots, k_{s-1}(t(m, s, p, r))=k_{s-2}(m) .
\end{gathered}
$$

3) а) Для каждого $s=1, \ldots, j(m)$ такого, что $k_{s}(m)>k_{s-1}(m)+1$

$$
\Delta \varphi_{\alpha(s), m}^{i}=0, \quad i=k_{s-1}(m)+1, \ldots, k_{s}(m)-1
$$

б) для каждого $s=1, \ldots, j(m)-1$

$$
\Delta \varphi_{\alpha(s), m}^{k_{s}(m)}(z)=\varphi_{\alpha(s+1), m}^{k_{s}(m)}(z), \quad z \in V_{\alpha(s+1)}(M-m+2)
$$


в) если $k_{s}(m)>s$, то для каждого $i=k_{s}(m), \ldots, n$ (при $i=k_{s}(m)$ последний член отсутствует) и всех $z \in V_{\alpha(s+1)}(M-m+2)$ имеем

$$
\begin{aligned}
\Delta h_{\alpha(s), m}^{i, 2} & (z)+\Delta h_{\alpha(s), m}^{i, 4}(z)=-\sum_{p} \sum_{r=k_{p-1}(m)+1}^{k_{p}(m)-1}(-1)^{s+1-p} \\
& \times\left[h_{\alpha(s+1), t(m, s+1, p, r)}^{i, 1}(z)+h_{\alpha(s+1), t(m, s+1, p, r)}^{i, 3}(z)\right] F_{r}(z) \\
& -\sum_{p} \sum_{r=k_{p-1}(m)+1}^{k_{p}(m)-1}(-1)^{s+1-p} \sum_{j=k_{s}(m)}^{i-1} \varphi_{\alpha(s+1), t(m, s+1, p, r)}^{i, j}(z) F_{j}(z) F_{r}(z)
\end{aligned}
$$

где суммирование по $p$ и индексы $t(m, s+1, p, r)$ определены в условии $2, \Gamma)$.

4) Сушествует выпуклый компакт $L_{m} \subset G$ такой, что $L_{m-1} \subset L_{m}, m>1$, и

а) $H_{L_{m}}(z)+(n(M-m)(M+11)+10) \varepsilon|z|<H_{G}(z), z \neq 0$;

б) $\int_{V_{\alpha(s), m}(M-m+2)}\left|h_{\alpha(s), m}^{i, 1}\right|^{2} \exp \left\{-2\left[H_{L_{m}}+H_{s, m}-H_{K_{i}}+(s-1) \psi\right]\right\} d \sigma<\infty$, где функция $\psi(z)$ определена вьше и $H_{s, m}(z)=\sum_{j=1, j \neq k_{1}(m), \ldots, k_{s-1}(m)}^{n} H_{K_{j}}(z)$;

в) $\int_{V_{\alpha(s), m}(M-m+2)}\left|\varphi_{\alpha(s), m}^{i, r}\right|^{2} \exp \left\{-2\left[H_{L_{m}}+H_{s, m}-H_{K_{i}}-H_{K_{r}}+s \psi\right]\right\} d \sigma<\infty$.

Итак, мы должны построить наборы $\left\{\varphi_{\alpha(s), m}^{i}\right\} \in \Phi_{m}$, удовлетворяющие условиям 1)-4). Однако перед этим мы хотим показать, что существование такого набора для $m=M$ влечет за собой существование необходимого нам набора функций $\varphi_{1}, \ldots, \varphi_{n}$.

Пусть набор $\left\{\varphi_{\alpha(s), M}^{i}\right\} \in \Phi_{M}$ удовлетворяет условиям 1)-4). Отметим вначале, что $j(M)=1$, т.е. $\left(k_{1}(M), \ldots, k_{n}(M)\right)$ совпадает с вектором $(n, \ldots, n)$. Таким образом, набор $\left\{\varphi_{\alpha(s), M}^{i}\right\} \in \Phi_{M}$ состоит из функций $\varphi_{j, M}^{i}, i, j=1, \ldots, n$. Согласно условию 2) имеем $\Delta \varphi_{j, M}^{i}=0 \forall i=1, \ldots, n-1$. Кроме того, все функции $\varphi_{j, M}^{0}$ являются сужением одной и той же функции $F / f$. Поэтому $\Delta \varphi_{j, M}^{0}=0$. Отсюда с учетом условия 1) получаем

$$
0=\Delta \varphi_{j, M}^{0}=\sum_{i=1}^{n} \Delta \varphi_{j, M}^{i}=\Delta \varphi_{j, M}^{n} .
$$

Следовательно, в силу определения кограничного оператора имеют место равенства

$$
\varphi_{j, M}^{i}(z)-\varphi_{k, M}^{i}(z)=0, \quad z \in V_{j}(2) \cap V_{k}(2), \quad i, j, k=1, \ldots, n .
$$

Это означает, что для всех $i=1, \ldots, n$ определена целая функция $\varphi_{i}$, которая на $V_{j}(2), j=1, \ldots, n$, совпадает с $\varphi_{j, M}^{i}$. Умножая обе части равенства из условия 1$)$ на $f$ и вспоминая определения функций $\varphi_{j, M}^{0}$ и $F_{i}$, получаем

$$
F(z) \equiv \varphi_{1}(z) f_{1}(z)+\cdots+\varphi_{n}(z) f_{n}(z)
$$

Остается показать, что $\varphi_{i} \in P_{G_{i}}, i=1, \ldots, n$, т.е. для $\varphi_{i}$ выполнены необходимые оценки. Для этого достаточно оценить функции $\varphi_{j, M}^{i}$, которые согласно условию 2) представляются в виде суммы $h_{j, M}^{i, k}, k=1, \ldots, 4$. Так как $k_{1-1}(M)=$ $k_{0}(M)=0=1-1$, то по условию $\left.2, \Gamma\right)$ функции $h_{j, M}^{i, 4}$ являются тождественным 
нулем. Поэтому, учитывая представления $h_{j, M}^{i, 2}$ и $h_{j, M}^{i, 3}$ из условий 2, б) и 2, в), достаточно оценить функции $h_{j, M}^{i, 1}, \varphi_{j, M}^{i, r} F_{r}$ и $\varphi_{j, M}^{k, i} F_{k}$.

По условию $4, б)$ для случая $m=M$ получаем

$$
\int_{V_{j}(2)}\left|h_{j, M}^{i, 1}(z)\right|^{2} \exp \left\{-2 H_{L_{M}}(z)-2 \sum_{p=1, p \neq i}^{n} H_{K_{p}}(z)\right\} d \sigma<\infty
$$

Точно так же условие 4,в) дает нам:

$$
\int_{V_{j}(2)}\left|\varphi_{j, M}^{i, r} F_{r}\right|^{2} \exp \left\{-2 H_{L_{M}}-2 \sum_{p=1, p \neq i, r}^{n} H_{K_{p}}-2 \psi-2 \ln \left|F_{r}\right|\right\} d \sigma<\infty
$$

Отсюда и из леммы 3.6 следует, что

$$
\int_{V_{j}(2)}\left|\varphi_{j, M}^{i, r}(z) F_{r}(z)\right|^{2} \exp \left\{-2 H_{L_{M}}(z)-2 \sum_{p=1, p \neq i}^{n} H_{K_{p}}(z)-16 \varepsilon|z|\right\} d \sigma<\infty
$$

Аналогичное неравенство имеет место, конечно, и для функций $\varphi_{j, M}^{k, i} F_{k}$. Используя теперь неравенство Коши-Буняковского для интегралов, уже нетрудно получить оценку

$$
\int_{V_{j}(2)}\left|\varphi_{j, M}^{i}(z)\right|^{2} \exp \left\{-2 H_{L_{M}}(z)-2 \sum_{p=1, p \neq i}^{n} H_{K_{p}}(z)-16 \varepsilon|z|\right\} d \sigma<\infty
$$

В силу аддитивности интеграла по множеству интегрирования и неотрицательности подынтегральной функции находим отсюда, что

$$
\int_{\mathbb{C}}\left|\varphi_{i}(z)\right|^{2} \exp \left\{-2 H_{L_{M}}(z)-2 \sum_{p=1, p \neq i}^{n} H_{K_{p}}(z)-16 \varepsilon|z|\right\} d \sigma<\infty
$$

Отсюда, используя неравенство о среднем для субгармонических функций и неравенство Коши-Буняковского для интегралов, получаем

$$
\begin{aligned}
\left|\varphi_{i}(y)\right| \leqslant & c \int_{B(y, 1)}\left|\varphi_{i}(z)\right| d \sigma \\
\leqslant & c\left[\int_{B(y, 1)}\left|\varphi_{i}(z)\right|^{2} \exp \left\{-2 H_{L_{M}}(z)-2 \sum_{p=1, p \neq i}^{n} H_{K_{p}}(z)-16 \varepsilon|z|\right\} d \sigma\right]^{\frac{1}{2}} \\
& \times\left[\int_{B(y, 1)} \exp \left\{2 H_{L_{M}}(z)+2 \sum_{p=1, p \neq i}^{n} H_{K_{p}}(z)+16 \varepsilon|z|\right\} d \sigma\right]^{\frac{1}{2}} \\
\leqslant & c_{1} \sup _{z \in B(y, 1)} \exp \left[H_{L_{M}}(z)+\sum_{p=1, p \neq i}^{n} H_{K_{p}}(z)+8 \varepsilon|z|\right]
\end{aligned}
$$


где $c_{1}>0$ не зависит от $y \in \mathbb{C}$. Используя теперь равномерную непрерывность опорных функций на окружности $\mathbb{S}$ и их положительную однородность (как и в $(3.10))$, из предыдущего уже нетрудно получить оценку

$$
\left|\varphi_{i}(y)\right| \leqslant c_{2} \exp \left[H_{L_{M}}(y)+\sum_{p=1, p \neq i}^{n} H_{K_{p}}(y)+9 \varepsilon|y|\right], \quad y \in \mathbb{C},
$$

где $c_{2}>0$ не зависит от $y$. Из условия 4, a) для случая $m=M$ следует, что

$H_{L_{M}}(z)+\sum_{p=1, p \neq i}^{n} H_{K_{p}}(z)+9 \varepsilon|z| \leqslant H_{G}(z)-\varepsilon|z|+\sum_{p=1, p \neq i}^{n} H_{K_{p}}(z)=H_{G_{i}}(z)-\varepsilon|z|$

для всех $z$. Это означает, что выпуклый компакт $T$, являюшийся замыканием $9 \varepsilon$-вздутия множества $L_{M}+\sum_{p=1, p \neq i}^{n} K_{p}$, лежит в $G$. По доказанному

$$
\left|\varphi_{i}(z)\right| \leqslant c_{2} \exp H_{T}(z), \quad z \in \mathbb{C} .
$$

Следовательно, $\varphi_{i} \in P_{G_{i}}, i=1, \ldots, n$.

Таким образом, нам остается доказать существование набора $\left\{\varphi_{\alpha(s), m}^{i}\right\} \in \Phi_{m}$, удовлетворяющего условиям 1$)-4$ ), для всех $m=1, \ldots, M$. Покажем вначале, что такой набор существует для $m=1$.

Из определения множества векторов $\Xi$ и правила их упорядочивания следует, что $k_{s}(1)=s$ для всех $s=1, \ldots, n$ и $j(1)=n$. Для каждых $i, j=1, \ldots, n$ определим функции

$$
\begin{aligned}
& \varphi_{i, 1}^{i}(z)=\frac{F(z)}{f_{i}(z)}, \quad z \in V_{i}(M+1), \\
& \varphi_{j, 1}^{i}(z)=0, \quad z \in V_{i}(M+1), \quad \forall j \neq i .
\end{aligned}
$$

Для $s=2, \ldots, n$ положим

$$
\varphi_{\alpha(s), 1}^{s-1}(z)=\Delta \varphi_{\alpha(s-1), 1}^{s-1}(z), \quad z \in V_{\alpha(s)}(M+1) .
$$

Пусть $i=s, \ldots, n$. Если $\varphi_{\alpha(s), 1}^{s-1}$ не равна тождественно нулю, то положим

$$
\varphi_{\alpha(s), 1}^{i}(z)=-\frac{\Delta \varphi_{\alpha(s-1), 1}^{i}(z)}{F_{s-1}(z)}, \quad z \in V_{\alpha(s)}(M+1) .
$$

В противном случае полагаем

$$
\varphi_{\alpha(s), 1}^{i}(z)=0, \quad z \in V_{\alpha(s)}(M+1) .
$$

Таким образом, при всех возможных (для $m=1$ ) значениях $s$ и $i$ определены функции $\varphi_{\alpha(s), 1}^{i}$. Покажем, что набор $\left\{\varphi_{\alpha(s), 1}^{i}\right\}$ принадлежит пространству $\Phi_{1}$. Для этого мы вначале докажем, что $\varphi_{\alpha(s), 1}^{i}$ тождественно равна нулю, если вектор $\alpha(s)$ не содержит компоненты, равной $i$. Согласно (3.11) это выполнено для $s=1$. Предположим, что это же выполнено и для всех $s<k$. 
Пусть теперь $s=k$. Из определений $\varphi_{\alpha(k), 1}^{i}$ и кограничного оператора имеем

$$
\varphi_{\alpha_{1}, \ldots, \alpha_{k} ; 1}^{i}(z)=\Theta(z) \sum_{j=1}^{k}(-1)^{j-1} \varphi_{\alpha_{1}, \ldots, \alpha_{j-1}, \alpha_{j+1}, \ldots, \alpha_{k} ; 1}^{i}(z),
$$

где $\Theta(z)=0$, либо $\Theta(z)=1$, либо $\Theta(z)=-1 / F_{k-1}(z), z \in V_{\alpha(k)}(M+1)$.

Пусть $\alpha_{j} \neq i$ для всех $j=1, \ldots, k$. Тогда по допущению индукции функция $\varphi_{\alpha_{1}, \ldots, \alpha_{j-1}, \alpha_{j+1}, \ldots, \alpha_{k} ; 1}^{i}$ тождественно равна нулю для любого $j=1, \ldots, k$. Поэтому $\varphi_{\alpha(k), 1}^{i}$ также является тождественным нулем. Это доказывает требуемое.

Покажем теперь, что функция $\varphi_{\alpha(s), 1}^{i}$ аналитична на множестве $V_{\alpha(s)}(M+1)$. Пусть вначале $s=1$. Согласно (3.11) для каждого $i=1, \ldots, n$ на $V_{i}(M+1)$ отлична от нуля только функция $\varphi_{i, 1}^{i}$. Отсутствие у нее аналитичности может быть вызвано лишь делением на $f_{i}$ при ее определении. Пусть $\Lambda=\left\{\lambda_{j}, m_{j}\right\}$-все обшие нули и их кратности функций $f_{1}, \ldots, f_{n}$. Из определения $q_{\delta}(z, x, \Lambda)$ и построения множества $V_{i}(M+1)$ следует, что последнее содержит только те нули функции $f_{i}$, которые являются членами последовательности $\left\{\lambda_{j}\right\}$. Причем кратность $\lambda_{j}$, попавшего в $V_{i}(M+1)$, как нуля $f_{i}$ равна $m_{j}$. Поскольку $F$ принадлежит множеству $I(D, \Lambda)$, которое состоит из функций, обрашаюшихся в нуль во всех точках $\lambda_{j} \mathrm{c}$ кратностью, не меньшей чем $m_{j}$, то функция $\varphi_{i, 1}^{i}=F / f_{i}$ аналитична на $V_{i}(M+1)$.

Предположим, что все функции $\varphi_{\alpha(s), 1}^{i}$ аналитичны на соответствуюших множествах при $s<k$, и пусть теперь $s=k$. Если $\varphi_{\alpha(k), 1}^{i} \equiv 0$, то доказывать нечего. Если же $\varphi_{\alpha(k), 1}^{i}$ не является тождественным нулем, то согласно ее определению не будет тождественным нулем и функция $\varphi_{\alpha(k), 1}^{k-1}$. По доказанному выше это означает, что вектор $\alpha(k)$ содержит компоненту, равную $k-1$. Но тогда верно включение $V_{\alpha(k)}(M+1) \subset V_{k-1}(M+1)$. Как отмечалось ранее, функция $F_{k-1}$ аналитическая и не имеет нулей на множестве $V_{k-1}(M+1)$, а следовательно, и на множестве $V_{\alpha(k)}(M+1)$. Поэтому деление на $F_{k-1}$ при определении функции $\varphi_{\alpha(k), 1}^{i}$ не может являться причиной неаналитичности последней. Кроме того, по допущению индукции функции $\varphi_{\alpha(k-1), 1}^{i}$ аналитические. Следовательно, аналитическими являются и функции $\Delta \varphi_{\alpha(k-1), 1}^{i}$. Все сказанное с учетом определения $\varphi_{\alpha(k), 1}^{i}$ означает, что $\varphi_{\alpha(k), 1}^{i}-$ аналитическая функция на множестве $V_{\alpha(k)}(M+1)$. Действительно, $\varphi_{\alpha(s), 1}^{i}$ - аналитическая функция в окрестности замыкания множества $V_{\alpha(s)}(M+1)$ (нетрудно видеть, что все приведенные выше рассуждения проходят и в этом случае).

Из построения $\varphi_{\alpha(s), 1}^{i}$ и определения кограничного оператора легко следует кососимметричность $\varphi_{\alpha(s), 1}^{i}$ по индексам $\alpha(s)=\left(\alpha_{1}, \ldots, \alpha_{s}\right)$. Таким образом, мы показали, что набор $\left\{\varphi_{\alpha(s), 1}^{i}\right\}$ принадлежит пространству $\Phi_{1}$. Проверим теперь выполнение условий 1)-4) для этого набора. Поскольку $k_{s}(1)=s$ для всех $s=1, \ldots, n$, то для проверки равенства из условия 1$)$ нужно показать, что

$$
\varphi_{\alpha(s), 1}^{s-1}(z)=\sum_{i=s}^{n} \varphi_{\alpha(s), 1}^{i}(z) F_{i}(z), \quad z \in V_{\alpha(s)}(M+1),
$$

для каждого $s=1, \ldots, n$. Пусть вначале $s=1$. В этом случае равенство (3.14) легко следует из (3.11) и определения функций $\varphi_{j, 1}^{0}$ и $F_{i}$. Предположим, что мы 
уже доказали (3.14) для всех $s<k$, и пусть теперь $s=k$. Если $\varphi_{\alpha(k), 1}^{k-1} \equiv 0$, то по построению $\varphi_{\alpha(k), 1}^{i}$ тождественно равны нулю для всех $i=k, \ldots, n$. В этом случае (3.14) выполнено тривиально. Пусть $\varphi_{\alpha(k), 1}^{k-1}$ не является тождественным нулем. Тогда $\varphi_{\alpha(k), 1}^{i}$ для всех $i=k, \ldots, n$ определяются по формуле (3.13). Coгласно допущению индукции (3.14) верно при $s=k-1$, т.е.

$$
\varphi_{\alpha(k-1), 1}^{k-2}(z)=\sum_{i=k-1}^{n} \varphi_{\alpha(k-1), 1}^{i}(z) F_{i}(z), \quad z \in V_{\alpha(k-1)}(M+1) .
$$

Если $k=2$, то, действуя оператором $\Delta$ на обе части этого равенства и вспоминая, что $\Delta \varphi_{j, 1}^{0}=0$, получаем

$$
0=\Delta \varphi_{j, 1}^{0}(z)=\sum_{i=1}^{n} \Delta \varphi_{j, 1}^{i}(z) F_{i}(z)
$$

Если же $k>2$, то точно так же с учетом свойства кограничного оператора $\left(\Delta^{2}=0\right)$ и равенства $(3.12)$ получаем

$$
0=\Delta^{2} \varphi_{\alpha(k-2), 1}^{k-2}(z)=\Delta \varphi_{\alpha(k-1), 1}^{k-2}(z)=\sum_{i=k-1}^{n} \Delta \varphi_{\alpha(k-1), 1}^{i}(z) F_{i}(z) .
$$

Элементарные преобразования двух последних соотношений дают равенство

$$
\Delta \varphi_{\alpha(k-1), 1}^{k-1}(z)=\sum_{i=k}^{n}\left[\frac{\Delta \varphi_{\alpha(k-1), 1}^{i}(z)}{F_{k-1}(z)}\right] F_{i}(z), \quad z \in V_{\alpha(k)}(M+1) .
$$

Вместе с (3.12) и (3.13) это обеспечивает вьполнение (3.14) для случая $s=k$. Таким образом, условие 1) выполнено для набора $\left\{\varphi_{\alpha(s), 1}^{i}\right\}$. Так как $s=k_{s}(1)=$ $k_{s-1}(1)+1$ для всех $s=1, \ldots, n$, то условие 3$)$ выполнено в силу (3.12). Для всех возможных $s$ и $i$ положим $h_{\alpha(s), 1}^{i, 1}=\varphi_{\alpha(s), 1}^{i}, h_{\alpha(s), 1}^{i, 2}=h_{\alpha(s), 1}^{i, 3}=h_{\alpha(s), 1}^{i, 4}=0$. Тогда в силу доказанного выше выполнено условие 2, a). Выполняются также и условия 3, б) -г), поскольку $s=k_{s}(1)=k_{s-1}(1)+1 \forall s=1, \ldots, n$.

Остается проверить условия 4, a) и 4, б). Требуемый в этих условиях компакт $L_{1}$ мы будем строить при помощи индукции. Мы докажем существование возрастающей последовательности компактов $L_{1,1} \subset \cdots \subset L_{1, n}$ из области $G$ таких, что для каждого $s=1, \ldots, n$ выполнены неравенства

$$
\begin{gathered}
H_{L_{1, s}}(z)+((n-s)(M+11)+n(M-1)(M+11)+10) \varepsilon|z|<H_{G}(z), \quad \begin{array}{r}
z \neq 0 \\
\int_{V_{\alpha(s)}(M+1)}\left|h_{\alpha(s), 1}^{i, 1}\right|^{2} \exp \left\{-2\left[H_{L_{1, s}}+\sum_{j=s, j \neq i}^{n} H_{K_{j}}+(s-1) \psi\right]\right\} d \sigma<\infty \\
i=s, \ldots, n .
\end{array}
\end{gathered}
$$

Предположим, что мы построили такую последовательность компактов. Тогда в качестве $L_{1}$ можно взять компакт $L_{1, n}$. Действительно, неравенство (3.15) при 
$s=n$ дает нам условие 4, а). Кроме того, из включения $L_{1, s} \subset L_{1, n}$ следует, что $H_{L_{1, s}}(z) \leqslant H_{L_{1, n}}(z), z \in \mathbb{C}$. Поэтому неравенство (3.16) сохранится, если компакт $L_{1, s}$ в нем заменить на $L_{1, n}$. А так как $k_{s}(1)=s \forall s=1, \ldots, n$, то (3.16) означает выполнение условия $4, \sigma)$.

Приступим теперь к построению компакта $L_{1,1}$. Согласно $(3.11)$ и определению $h_{j, 1}^{i, 1}$ неравенство (3.16) нужно проверить лишь для функций $h_{j, 1}^{i, 1}=F / f_{i}$, $i=1, \ldots, n$. Пусть $z \in V_{i}(M+1) \backslash B\left(0, R_{0}\right)$ такое, что $z /|z| \notin X(M+1)$, где $X(d)$ - множество, определенное перед леммой 3.3. Тогда, как уже отмечалось выше, найдутся $l$ и $k$ такие, что $z \in U_{k, l}^{i}(M+1) \subset B\left(z_{k, l},(M+1) \delta_{l}\left|z_{k, l}\right|\right)$. (Здесь мы используем те же обозначения, что и при построении покрытия $V_{1}(d), \ldots, V_{n}(d)$.) Согласно (2.2) и (3.8) имеем

$$
\begin{gathered}
\left|\frac{F(y)}{q_{2(M+1) \delta_{l}}\left(y, z_{k, l}, \Lambda\right)}\right| \leqslant|F(y)| \leqslant \tilde{A} \exp \left\{\sum_{j=0}^{n} H_{K_{j}}(y)\right\} \\
y \in \partial B\left(z_{k, l}, 4(M+1) \delta_{l}\left|z_{k, l}\right|\right)
\end{gathered}
$$

В силу выбора $\delta_{l}$ выполнено неравенство $(M+1) \delta_{l}<1 / 2$. Следовательно,

$$
B\left(z_{k, l}, 4(M+1) \delta_{l}\left|z_{k, l}\right|\right) \subset B\left(z, 10(M+1) \delta_{l}|z|\right) .
$$

Поэтому, используя неравенства (3.3) (напомним, что $h_{f_{i}} \equiv H_{K_{i}}$ ) и (3.10), из предыдушего получаем

$$
\begin{gathered}
\left|\frac{F(y)}{q_{2(M+1) \delta_{l}}\left(y, z_{k, l}, \Lambda\right)}\right| \leqslant \tilde{A} \exp \left\{\sum_{j=0}^{n} H_{K_{j}}(z)+2 \varepsilon|z|\right\} \\
y \in \partial B\left(z_{k, l}, 4(M+1) \delta_{l}\left|z_{k, l}\right|\right) .
\end{gathered}
$$

Так как $F \in I(D, \Lambda)$, то согласно определениям $I(D, \Lambda)$ и $q_{2(M+1) \delta_{l}}\left(y, z_{k, l}, \Lambda\right)$ частное $F(y) / q_{2(M+1) \delta_{l}}\left(y, z_{k, l}, \Lambda\right)$ является аналитической функцией (и даже целой). Следовательно, по принципу максимума последнее неравенство выполнено всюду в круге $B\left(z_{k, l}, 4(M+1) \delta_{l}\left|z_{k, l}\right|\right)$ и, в частности, в точке $z$. Тогда, учитывая определение $U_{k, l}^{i}(M+1)$ и тождество $h_{f_{i}} \equiv H_{K_{i}}$, получаем

$$
\begin{aligned}
\left|h_{i, 1}^{i, 1}(z)\right| & =\left|\varphi_{i, 1}^{i}(z)\right|=\left|F(z) f_{i}^{-1}(z)\right|=\frac{\left|F(z) q_{2(M+1) \delta_{l}}^{-1}\left(z, z_{k, l}, \Lambda\right)\right|}{\left|f_{i}(z) q_{2(M+1) \delta_{l}}^{-1}\left(z, z_{k, l}, \Lambda\right)\right|} \\
& \leqslant \tilde{A} \exp \left[\sum_{j=0, j \neq i}^{n} H_{K_{j}}(z)+(M+3) \varepsilon|z|\right] .
\end{aligned}
$$

Совершенно аналогично доказывается, что для точек $z \in V_{i}(M+1) \backslash B\left(0, R_{0}\right)$ таких, что $z /|z| \in X(M+1)$, найдутся числа $\tilde{A}_{1}>0$ и $B>0$ (не зависящие от $z$ ) такие, что верно неравенство

$$
\left|h_{i, 1}^{i, 1}(z)\right| \leqslant \tilde{A}_{1} \exp \left[B|z|+\sum_{j=1, j \neq i}^{n} H_{K_{j}}(z)\right] .
$$


Здесь в отличие от предыдушего мы ведем суммирование с $j=1$, поскольку функция $H_{K_{0}}$ нас в данном случае не интересует, а ее рост компенсируется величиной числа $B$.

Далее для построения $L_{1,1}$ мы хотим применить лемму 3.2. В качестве рассматриваемых там области, компакта и числа $a>0$ возьмем соответственно область $G$, компакт, являющийся замыканием $K_{0}+B(0,(M+4) \varepsilon)$, который согласно (3.9) лежит в $G$, и число $[(n-1)(M+11)+n(M-1)(M+11)+10] \varepsilon$. Проверим вьполнение условий этой леммы. Указанная в них оценка следует из (3.9). В качестве $X \subset \mathbb{S}$ возьмем $X(M+1)$. По построению $X(M+1)$ компактно принадлежит углу, порожденному множеством $\mathbb{S} \backslash Z$, а роль $Z$ в нашей теореме играет замыкание множества $\mathbb{S} \backslash I_{G}$. Следовательно, $X(M+1)$ компактно принадлежит int $I_{G}$. Таким образом, мы вправе применить лемму 3.2. Согласно ей для числа $A=B+\varepsilon$ в области $G$ найдется выпуклый компакт $L_{1,1}$, содержащий $K_{0}+B(0,(M+4) \varepsilon)$ и такой, что верны неравенства

$$
\begin{gathered}
H_{L_{1,1}}(z)+((n-1)(M+11)+n(M-1)(M+11)+10) \varepsilon|z|<H_{G}(z), \quad z \neq 0, \\
H_{L_{1,1}}(z)>(B+\varepsilon)|z| \quad \forall z: z /|z| \in X(M+1) .
\end{gathered}
$$

Первое из этих неравенств дает нам (3.15). Включение $K_{0}+B(0,(M+4) \varepsilon) \subset L_{1,1}$ означает, что

$$
H_{K_{0}}(z)+(M+4) \varepsilon|z| \leqslant H_{L_{1,1}}(z) \quad \forall z \in \mathbb{C} .
$$

Из последних неравенств и полученных выше оценок на $h_{i, 1}^{i, 1}$ следует, что

$$
\left|h_{i, 1}^{i, 1}(z)\right| \leqslant \tilde{A}_{2} \exp \left[H_{L_{1,1}}(z)-\varepsilon|z|+\sum_{j=1, j \neq i}^{n} H_{K_{j}}(z)\right], \quad z \in V_{i}(M+1) \backslash B\left(0, R_{0}\right) .
$$

Ранее отмечалось, что функция $\varphi_{i, 1}^{i} \equiv h_{i, 1}^{i, 1}$ аналитична на замыкании множества $X(M+1)$. Следовательно, она ограничена на $X(M+1) \cap B\left(0, R_{0}\right)$. Поэтому можно считать, что последнее неравенство выполнено в каждой точке $z \in X(M+1)$. Но тогда конечен интеграл

$$
\int_{V_{i}(M+1)}\left|h_{i, 1}^{i, 1}(z)\right|^{2} \exp \left\{-2\left[H_{L_{1,1}}(z)+\sum_{j=1, j \neq i}^{n} H_{K_{j}}(z)\right]\right\} d \sigma
$$

т.е. вьполнено (3.16).

Предположим, что мы уже построили компакты $L_{1,1}, \ldots, L_{1, s-1}$ с нужными свойствами. Докажем теперь существование $L_{1, s}$. Достаточно проверить (3.16) лишш для функций $h_{\alpha(s), 1}^{i, 1} \equiv \varphi_{\alpha(s), 1}^{i}, i=s, \ldots, n$, не равных тождественно нулю. Но в этом случае согласно (3.13) имеем

$$
h_{\alpha(s), 1}^{i, 1}(z)=-\frac{\Delta \varphi_{\alpha(s-1), 1}^{i}(z)}{F_{s-1}(z)}=-\frac{\Delta h_{\alpha(s-1), 1}^{i, 1}(z)}{F_{s-1}(z)}, \quad z \in V_{\alpha(s)}(M+1) .
$$

Кроме того, функция $\varphi_{\alpha(s), 1}^{s-1}$ не является тождественным нулем. Согласно доказанному выше это означает, что вектор $\alpha(s)$ должен содержать компоненту, 
равную $s-1$. Следовательно, $V_{\alpha(s)}(M+1) \subset V_{s-1}(M+1)$. В силу последнего равенства $h_{\alpha(s), 1}^{i, 1} F_{s-1}=-\Delta h_{\alpha(s-1), 1}^{i, 1}$, т.е. $h_{\alpha(s), 1}^{i, 1} F_{s-1}$ линейно выражается через $h_{\alpha(s-1), 1}^{i, 1}$. По допущению индукции $h_{\alpha(s-1), 1}^{i, 1}$ удовлетворяют условию (3.16), переписанному для индекса $s-1$. Поэтому, используя неравенство Коши-Буняковского для интегралов, нетрудно показать, что

$$
\int_{V_{\alpha(s)}(M+1)}\left|h_{\alpha(s), 1}^{i, 1} F_{s-1}\right|^{2} \exp \left\{-2\left[H_{L_{1, s-1}}+\sum_{j=s, j \neq i}^{n} H_{K_{j}}+(s-2) \psi\right]\right\} d \sigma<\infty .
$$

Пусть $\Theta>0$ - то же, что и в лемме 3.5. Положим

$$
A=(M+1) \Theta+\max _{y \in \mathbb{S}}\left|H_{L_{1, s-1}}(y)\right|+\max _{y \in \mathbb{S}}\left|H_{K_{s-1}}(y)\right| .
$$

Используем вновь лемму 3.2 для области $G$, компакта $K$, являющегося замыканием множества $L_{1, s-1}+B(0,(M+1) \varepsilon)$, и числа $a=((n-s)(M+11)+n(M-1) \times$ $(M+11)+10) \varepsilon$. Согласно условию $(3.15)$, переписанному для индекса $s-1$, компакт $K$ удовлетворяет требуемому в лемме 3.2 неравенству. Тогда по утверждению этой леммы найдется компакт $L_{1, s} \subset G$, удовлетворяюший неравенству (3.15) и такой, что

$$
\begin{gathered}
H_{L_{1, s-1}}(z)+(M+1) \varepsilon|z| \leqslant H_{L_{1, s}}(z) \quad \forall z \quad\left(\text { т.е. } L_{1, s-1}+B(0,(M+1) \varepsilon) \subset L_{1, s}\right) ; \\
H_{L_{1, s}}(z) \geqslant A|z| \quad \forall z: z /|z| \in X(M+1) .
\end{gathered}
$$

Отсюда и из интегральной оценки на $h_{\alpha(s), 1}^{i, 1} F_{s-1}$ с учетом леммы 3.5 легко следует неравенство (3.16). Таким образом, мы построили набор $\left\{\varphi_{\alpha(s), 1}^{i}\right\} \in \Phi_{1}$, удовлетворяюший условиям 1)-4). Допустим, что аналогичные наборы $\left\{\varphi_{\alpha(s), k}^{i}\right\} \in \Phi_{k}$ найдены для всех номеров $k \leqslant m$. Построим теперь набор $\left\{\varphi_{\alpha(s), m+1}^{i}\right\} \in \Phi_{m+1}$. Прежде всего напомним, как определяется вектор $\left(k_{1}(m+1), \ldots, k_{n}(m+1)\right)$ через вектор $\left(k_{1}(m), \ldots, k_{n}(m)\right)$. Имеют место равенства:

$$
\begin{gathered}
j(m+1)=j(m)+n-k_{j(m)-1}(m)-2, \\
k_{s}(m+1)=k_{s}(m) \quad \forall s=0, \ldots, j(m)-2, \\
k_{j(m)-2+p}(m+1)=k_{j(m)-1}(m)+p \quad \forall p=1, \ldots, n-k_{j(m)-1}(m) .
\end{gathered}
$$

Приступим теперь к последовательному построению функций $\varphi_{\alpha(s), m+1}^{i}$. Для каждого $s=1, \ldots, j(m)-2$ и всех возможных $i, j, r$ положим

$$
\begin{gathered}
\varphi_{\alpha(s), m+1}^{i}(z)=\varphi_{\alpha(s), m}^{i}(z), \quad h_{\alpha(s), m+1}^{i, j}(z)=h_{\alpha(s), m}^{i, j}(z) \\
\varphi_{\alpha(s), m+1}^{i, r}(z)=\varphi_{\alpha(s), m}^{i, r}(z), \quad z \in V_{\alpha(s)}(M-m+1) .
\end{gathered}
$$

Чтобы определить функции $\varphi_{\alpha(j(m)-1), m+1}^{i}$, нам необходимо сделать некоторые дополнительные построения, основанные на решении $\bar{\partial}$-задачи с интегральными оценками. Через $j, \alpha(s)$ будем обозначать набор индексов $\left(j, \alpha_{1}, \ldots, \alpha_{s}\right)$. Пусть 
$E_{1}(z), \ldots, E_{n}(z)$ - разбиение единицы, построенное в лемме 3.4 для покрытия $V_{1}(M-m+2), \ldots, V_{n}(M-m+2)$ комплексной плоскости. Положим

$$
\begin{gathered}
\widetilde{\Theta}_{j, \alpha(j(m)-1)}^{i}(z)=\left\{\begin{array}{l}
E_{j}(z) \varphi_{j, \alpha(j(m)-1), m}^{i}(z), \quad z \in V_{j, \alpha(j(m)-1)}(M-m+2), \\
0, \quad z \in V_{\alpha(j(m)-1)}(M-m+2) \backslash V_{j, \alpha(j(m)-1)}(M-m+2),
\end{array}\right. \\
\Theta_{\alpha(j(m)-1)}^{i}(z)=\sum_{j=1}^{n} \widetilde{\Theta}_{j, \alpha(j(m)-1)}^{i}(z), \\
z \in V_{\alpha(j(m)-1)}(M-m+2), \quad i=k_{j(m)-1}(m)+1, \ldots, n .
\end{gathered}
$$

Функции $\widetilde{\Theta}_{j, \alpha(j(m)-1)}^{i}(z)$, а вместе с ними и $\Theta_{\alpha(j(m)-1)}^{i}(z)$ бесконечно дифференцируемы, поскольку $E_{j}(z)$ обращаются в нуль в окрестности границы множества $V_{j}(M-m+2)$. По допушению индукции $\varphi_{\alpha(s), m}^{i}$ удовлетворяют условиям 1$\left.)-4\right)$. Согласно равенству $\Delta^{2}=0$ и условию 3, б) $\Delta \varphi_{\alpha(j(m)), m}^{k_{j(m)-1}(m)}=0$. Кроме того, по условию 3, а) $\Delta \varphi_{\alpha(j(m)), m}^{i}=0 \forall i=k_{j(m)-1}(m)+1, \ldots, k_{j(m)}(m)-1=n-1$. Следовательно, по условию 1) получаем

$$
0=\sum \Delta \varphi_{\alpha(j(m)), m}^{i}=\Delta \varphi_{\alpha(j(m)), m}^{n} .
$$

Таким образом, коцепи $\varphi_{\alpha(j(m)), m}^{i}, i=k_{j(m)-1}(m)+1, \ldots, n$, являются коциклами. Но тогда, как обычно (см., например, $[16$, с. 308]), получаем

$$
\Delta \Theta_{\alpha(j(m)-1)}^{i}=\varphi_{\alpha(j(m)), m}^{i}, \quad i=k_{j(m)-1}(m)+1, \ldots, n .
$$

Пусть $L \subset G$ - вьпуклый компакт, построенньй по лемме 3.2 для области $G$, компакта, являюшегося замыканием суммы $L_{m}+B(0,(M-m+11) \varepsilon)$, множества $X(M-m+2) \subset \mathbb{S}$, а также чисел $A=\tilde{b}+\max _{z \in \mathbb{S}}\left|H_{L_{m}}(z)\right|$ и $a=((n-1)(M+$ $11)+1+n(M-m-1)(M+11)+10) \varepsilon(\tilde{b}-$ то же, что и в оценке для производной $E_{i}(z)$ в лемме 3.4$)$. Мы вправе применить здесь эту лемму, поскольку для компакта $L_{m}$ выполнено условие 4, a). Согласно утверждению леммы 3.2 для каждого $z \neq 0$ имеют место неравенства

$$
\begin{aligned}
H_{L}(z)+ & ((n-1)(M+11)+1+n(M-m-1)(M+11)+10) \varepsilon|z|<H_{G}(z), \\
& H_{L}(z)>\left(\tilde{b}+\max _{z \in \mathbb{S}}\left|H_{L_{m}}(z)\right|\right)|z|, \quad z /|z| \in X(M-m+2)
\end{aligned}
$$

и верно включение $L_{m}+B(0,(M-m+11) \varepsilon) \subset L$, т.е.

$$
H_{L_{m}}(z)+(M-m+11) \varepsilon|z| \leqslant H_{L}(z) \quad \forall z .
$$

Из условий $4, \sigma)$ и 4, в), как и для функций $\varphi_{j, M}^{i}$, следует, что

$$
\begin{aligned}
& \int_{V_{\alpha(j(m))}(M-m+2)}\left|\varphi_{\alpha(j(m)), m}^{i}\right|^{2} \\
& \quad \times \exp \left\{-2\left[H_{L_{m}}+H_{j(m), m}-H_{K_{i}}+(j(m)-1) \psi\right]\right\} d \sigma<\infty .
\end{aligned}
$$


Это же неравенство будет, очевидно, верньм, если $\varphi_{\alpha(j(m)), m}^{i}$ в нем заменить на $\widetilde{\Theta}_{\alpha(j(m))}^{i}$. А поскольку $\widetilde{\Theta}_{\alpha(j(m))}^{i}$ равна нулю вне $V_{\alpha(j(m))}(M-m+2)$, то $(3.21)$ будет верно, если $\varphi_{\alpha(j(m)), m}^{i}$ и $V_{\alpha(j(m))}(M-m+2)$ заменить соответственно на $\widetilde{\Theta}_{j, \alpha(j(m)-1)}^{i}$ и $V_{\alpha(j(m)-1)}(M-m+2)$. Но тогда, используя неравенство Коши-Буняковского для интегралов и включение $L_{m} \subset L$, нетрудно видеть, что

$$
\begin{gathered}
\int_{V_{\alpha(j(m)-1)}(M-m+2)}\left|\Theta_{\alpha(j(m)-1)}^{i}\right|^{2} \\
\times \exp \left\{-2\left[H_{L}+H_{j(m), m}-H_{K_{i}}+(j(m)-1) \psi\right]\right\} d \sigma<\infty \\
i=k_{j(m)-1}(m)+1, \ldots, n
\end{gathered}
$$

Для каждого $i=k_{j(m)-1}(m)+1, \ldots, n$ положим

$$
Y_{\alpha(j(m)-1)}^{i}(z)=\frac{d \Theta_{\alpha(j(m)-1)}^{i}(z)}{d \bar{z}}, \quad z \in V_{\alpha(j(m)-1)}(M-m+2)
$$

$\Phi$ ункции $\varphi_{\alpha(j(m)), m}^{i}$ аналитические, поэтому $Y_{\alpha(j(m)-1)}^{i}$ можно определить по тем же формулам, что и $\Theta_{\alpha(j(m)-1)}^{i}$, с единственной заменой $E_{j}$ на $d E_{i} / d \bar{z}$. Верно также неравенство

$$
\begin{aligned}
& \int_{V_{\alpha(j(m)-1)}(M-m+2)}\left|Y_{\alpha(j(m)-1)}^{i}\right|^{2} \\
& \quad \times \exp \left\{-2\left[H_{L}+H_{j(m), m}-H_{K_{i}}+(j(m)-1) \psi\right]\right\} d \sigma<\infty .
\end{aligned}
$$

Действительно, из (3.19), (3.20) и оценок на $\left|d E_{i} / d \bar{z}\right|$ в лемме 3.4 следует, что

$$
\ln \left|\frac{d E_{i}(z)}{d \bar{z}}\right|+H_{L_{m}}(z) \leqslant H_{L}(z)+\ln \tilde{c} .
$$

Отсюда и из (3.21), как и выше, получаем (3.23).

Кограничный оператор и оператор дифференцирования перестановочны друг с другом. Поэтому из (3.17), аналитичности функций $\varphi_{\alpha(j(m)), m}^{i}$ и определения $Y_{\alpha(j(m)-1)}^{i}$ следует, что $\Delta Y_{\alpha(j(m)-1)}^{i}=0$, т.е. $Y_{\alpha(j(m)-1)}^{i}$ - коцикл для всех $i=$ $k_{j(m)-1}(m)+1, \ldots, n$.

Далее мы разберем две ситуации: I) $j(m)-1>1$; II) $j(m)-1=1$.

Пусть вначале $j(m)-1>1$. В этом случае мы определим еще коцепи $Q_{\alpha(j(m)-2)}^{i}$, $i=k_{j(m)-1}(m)+1, \ldots, n$, по формулам

$$
\begin{aligned}
\widetilde{Q}_{j, \alpha(j(m)-2)}^{i}(z) & =\left\{\begin{array}{c}
E_{j}(z) Y_{j, \alpha(j(m)-2), m}^{i}(z), \quad z \in V_{j, \alpha(j(m)-2)}(M-m+2), \\
0, \quad z \in V_{\alpha(j(m)-2)}(M-m+2) \backslash V_{j, \alpha(j(m)-2)}(M-m+2),
\end{array}\right. \\
Q_{\alpha(j(m)-2)}^{i}(z) & =\sum_{j=1}^{n} \widetilde{Q}_{j, \alpha(j(m)-2)}^{i}(z), \quad z \in V_{\alpha(j(m)-2)}(M-m+2) .
\end{aligned}
$$


Как и выше, функции $Q_{\alpha(j(m)-2)}^{i}$ бесконечно дифференцируемы, а поскольку $Y_{\alpha(j(m)-1)}^{i}-$ коцикл, то (см. [16, с. 308]) имеют место равенства

$$
\Delta Q_{\alpha(j(m)-2)}^{i}=Y_{\alpha(j(m)-1)}^{i}, \quad i=k_{j(m)-1}(m)+1, \ldots, n .
$$

Пусть $\widetilde{E}_{\alpha(j(m)-2)}$ - функция, построенная в лемме 3.1 , где в качестве $\Omega$ и $K$ нужно взять соответственно $V_{\alpha(j(m)-2)}(M-m+2)$ и замыкание множества $V_{\alpha(j(m)-2)}(M-m+1)$ (это можно сделать в силу леммы 3.3$)$. При этом можно считать, что функции $\widetilde{E}_{\alpha(j(m)-2)}$ и $\widetilde{E}_{\beta(j(m)-2)}$, у которых векторы $\alpha(j(m)-2)$ и $\beta(j(m)-2)$ получаются друг из друга перестановкой компонент, равны. Тогда $\widetilde{E}_{\alpha(j(m)-2)} Q_{\alpha(j(m)-2)}^{i}$ кососимметричны по индексам $\alpha(j(m)-2)$. Кроме того, $\widetilde{E}_{\alpha(j(m)-2)} Q_{\alpha(j(m)-2)}^{i} \in C^{\infty}(\mathbb{C})$, поскольку функция $\widetilde{E}_{\alpha(j(m)-2)}$ равна нулю в окрестности границы множества $V_{\alpha(j(m)-2)}(M-m+2)$ и вне его. Из (3.23) и определения $Q_{\alpha(j(m)-2)}^{i}$, как и вьше, следует, что

$\int_{\mathbb{C}}\left|\widetilde{E}_{\alpha(j(m)-2)}^{i} Q_{\alpha(j(m)-2)}^{i}\right|^{2} \exp \left\{-2\left[H_{L}+H_{j(m), m}-H_{K_{i}}+(j(m)-1) \psi\right]\right\} d \sigma<\infty$.

Поэтому согласно [17, теорема 4.4.2] сушествует решение $T_{\alpha(j(m)-2)}^{i}$ уравнения

$$
\frac{d T_{\alpha(j(m)-2)}^{i}}{d \bar{z}}=\widetilde{E}_{\alpha(j(m)-2)} Q_{\alpha(j(m)-2)}^{i},
$$

принадлежащее $C^{\infty}(\mathbb{C})$ и удовлетворяюшее неравенству

$$
\int_{\mathbb{C}}\left|T_{\alpha(j(m)-2)}^{i}\right|^{2} \exp \left\{-2\left[H_{L_{m+1,1}}+H_{j(m), m}-H_{K_{i}}+(j(m)-1) \psi\right]\right\} d \sigma<\infty,
$$

где $L_{m+1,1}$ - замыкание множества $L+B(0, \varepsilon)$. При этом, поскольку набор $\widetilde{E}_{\alpha(j(m)-2)} Q_{\alpha(j(m)-2)}^{i}$ является коцепью для каждого $i=k_{j(m)-1}(m)+1, \ldots, n$, то $T_{\alpha(j(m)-2)}^{i}$ также можно считать коцепю. Положим

$A_{\alpha(j(m)-1)}^{i}(z)=\Theta_{\alpha(j(m)-1)}^{i}(z)-\Delta T_{\alpha(j(m)-2)}^{i}(z), \quad z \in V_{\alpha(j(m)-2)}(M-m+1)$.

Так как $\widetilde{E}_{\alpha(j(m)-2)}$ равна единице на множестве $V_{\alpha(j(m)-2)}(M-m+1)$, то из $(3.24)$, $(3.25)$ и определения $Y_{\alpha(j(m)-1)}^{i}$ получаем

$$
\begin{aligned}
& \frac{d A_{\alpha(j(m)-1)}^{i}(z)}{d \bar{z}}=\frac{d \Theta_{\alpha(j(m)-1)}^{i}(z)}{d \bar{z}}-\Delta\left[\frac{d T_{\alpha(j(m)-2)}^{i}(z)}{d \bar{z}}\right] \\
& =Y_{\alpha(j(m)-1)}^{i}(z)-\Delta Q_{\alpha(j(m)-2)}^{i}(z)=0, \quad z \in V_{\alpha(j(m)-2)}(M-m+1),
\end{aligned}
$$

т.е. функция $A_{\alpha(j(m)-1)}^{i}$ аналитична на множестве $V_{\alpha(j(m)-2)}(M-m+1)$. Поскольку $L \subset L_{m+1,1}$, то из $(3.22)$ и (3.26) следует, что

$$
\begin{aligned}
& \int_{V_{\alpha(j(m)-1)}(M-m+1)}\left|A_{\alpha(j(m)-1)}^{i}\right|^{2} \\
& \quad \times \exp \left\{-2\left[H_{L_{m+1,1}}+H_{j(m), m}-H_{K_{i}}+(j(m)-1) \psi\right]\right\} d \sigma<\infty .
\end{aligned}
$$


Равенство $\Delta^{2}=0$ дает нам: $\Delta A_{\alpha(j(m)-1)}^{i}=\Delta \Theta_{\alpha(j(m)-1)}^{i}$. Тогда в силу $(3.17)$ имеем

$$
\Delta A_{\alpha(j(m)-1)}^{i}=\varphi_{\alpha(j(m)), m}^{i}, \quad i=k_{j(m)-1}(m)+1, \ldots, n .
$$

Итак, мы полностью разобрали случай, когда $j(m)-1>1$.

Пусть теперь $j(m)-1=1$. Чуть выше мы показали, что $Y_{\alpha(j(m)-1)}^{i}-$ коцикл, т.е. в нашем случае $Y_{k}^{i}(z)-Y_{j}^{i}(z)=0 \quad \forall z \in V_{k}(M-m+2) \cap V_{j}(M-m+2)$. Поэтому для любого $i=k_{j(m)-1}(m)+1, \ldots, n$ определена функция $Y^{i} \in C^{\infty}(\mathbb{C})$, которая на множестве $V_{j}(M-m+2)$ совпадает с $Y_{j}^{i}$. В силу $(3.23)$

$$
\int_{\mathbb{C}}\left|Y^{i}\right|^{2} \exp \left\{-2\left[H_{L}+H_{j(m), m}-H_{K_{i}}+(j(m)-1) \psi\right]\right\} d \sigma<\infty .
$$

Тогда по $\left[17\right.$, теорема 4.4.2] сушествует решение $T^{i} \in C^{\infty}(\mathbb{C})$ уравнения

$$
\frac{d T^{i}(z)}{d \bar{z}}=Y^{i}(z)
$$

удовлетворяюшее неравенству

$$
\int_{\mathbb{C}}\left|T^{i}\right|^{2} \exp \left\{-2\left[H_{L_{m+1,1}}+H_{j(m), m}-H_{K_{i}}+(j(m)-1) \psi\right]\right\} d \sigma<\infty .
$$

Положим $A_{\alpha(j(m)-1)}^{i}=\Theta_{\alpha(j(m)-1)}^{i}-T^{i}$. В силу (3.29) и определения $Y_{\alpha(j(m)-1)}^{i}$ функция $A_{\alpha(j(m)-1)}^{i}$ аналитична на множестве $V_{\alpha(j(m)-1)}(M-m+2)$. Так как $L \subset L_{m+1,1}$, то из (3.22) и (3.30) следует, что $A_{\alpha(j(m)-1)}^{i}$ удовлетворяет (3.27). Функция $T^{i}$ одна и та же для всех индексов $\alpha(j(m)-1)$. Поэтому $\Delta T^{i}=0$ и $\Delta A_{\alpha(j(m)-1)}^{i}=\Delta \Theta_{\alpha(j(m)-1)}^{i}$. Отсюда с учетом (3.17) получаем (3.28).

Мы закончили необходимые построения и можем завершить теперь определение набора $\left\{\varphi_{\alpha(s), m+1}^{i}\right\}$. Для всех $i=k_{j(m)-1}(m)+1=k_{j(m)-1}(m+1), \ldots, n$ положим

$$
\varphi_{\alpha(j(m)-1), m+1}^{i, k_{j(m)-1}(m+1)-1}=A_{\alpha(j(m)-1)}^{i} .
$$

Для всех остальных возможных $i$ и $r$ пусть $\varphi_{\alpha(j(m)-1), m+1}^{i, r}=\varphi_{\alpha(j(m)-1), m}^{i, r}$. Это определение корректно, поскольку $k_{j(m)-2}(m)=k_{j(m)-2}(m+1)$ и $k_{j(m)-1}(m)+1=k_{j(m)-1}(m+1)$. Кроме того, пусть $h_{\alpha(j(m)-1), m+1}^{i, 1}=$ $h_{\alpha(j(m)-1), m}^{i, 1}$, ф функции $h_{\alpha(j(m)-1), m+1}^{i, j}, j=2,3,4, i=k_{j(m)-2}(m+1)+1, \ldots, n$, определены согласно формулам из условий $2, \sigma)-\Gamma)$, переписанным для индекса $m+1$. Наконец, положим

$$
\begin{aligned}
& \varphi_{\alpha(j(m)-1), m+1}^{k_{j(m)-2}(m+1)}=\varphi_{\alpha(j(m)-1), m}^{k_{j(m)-2}(m)}, \\
& \varphi_{\alpha(j(m)-1), m+1}^{i}=\sum_{j=1}^{4} h_{\alpha(j(m)-1), m+1}^{i, j}, \quad i=k_{j(m)-2}(m+1)+1, \ldots, n .
\end{aligned}
$$

Таким образом, функции $\varphi_{\alpha(j(m)-1), m+1}^{i}$ полностью определены. Если будет $k_{j(m)-1}(m)=n-1$, т.е. $j(m+1)=j(m)-1$, то построение закончено. В противном случае для всех $s=j(m), \ldots, j(m+1)$ и $i=k_{s-1}(m+1)+1, \ldots, n$ пусть

$$
h_{\alpha(s), m+1}^{i, 2}=h_{\alpha(s), m+1}^{i, 3}=0,
$$


а функции $h_{\alpha(s), m+1}^{i, 4}$ определены по формуле из условия $\left.2, \Gamma\right)$, переписанного для индекса $m+1$. Для каждого $s=j(m), \ldots, j(m+1)$ положим также

$$
\begin{gathered}
\varphi_{\alpha(s), m+1}^{k_{s-1}(m+1)}=\Delta \varphi_{\alpha(s-1), m+1}^{k_{s-1}(m+1)} \\
\varphi_{\alpha(s), m+1}^{i}=\sum_{j=1}^{4} h_{\alpha(s), m+1}^{i, j}, \quad i=k_{s-1}(m+1)+1, \ldots, n
\end{gathered}
$$

Тогда нам остается определить лишь функции $h_{\alpha(s), m+1}^{i, 1}, s=j(m), \ldots, j(m+1)$, $i=k_{s-1}(m+1)+1, \ldots, n$. Если

$$
\Delta h_{\alpha(s-1), m+1}^{k_{s-1}(m+1), 1}-\sum_{p} \sum_{r=k_{p-1}(m+1)+1}^{k_{p}(m+1)-1}(-1)^{s-p} h_{\alpha(s), t(m+1, s, p, r)}^{k_{s-1}(m+1), 1} F_{r} \equiv 0
$$

(суммирование по $p$ и номера $t(m+1, s, p, r)$ определены в условии $2, \Gamma)$ ), то полагаем $h_{\alpha(s), m+1}^{i, 1} \equiv 0, i=k_{s-1}(m+1)+1, \ldots, n$. В противном случае пусть

$$
\begin{aligned}
h_{\alpha(s), m+1}^{i, 1}= & -\left[\Delta h_{\alpha(s-1), m+1}^{i, 1}\right. \\
& -\sum_{p} \sum_{\substack{r=k_{p-1}(m+1)+1 \\
k_{p}(m+1)-1}}^{i=k_{s-1}(m+1)+1, \ldots, n .} .
\end{aligned}
$$

Таким образом, набор $\left\{\varphi_{\alpha(s), m+1}^{i}\right\}$ полностью определен. Теперь мы должны проверить, что $\left\{\varphi_{\alpha(s), m+1}^{i}\right\} \in \Phi_{m+1}$ и вьполнены условия 1)-4). По построению необходимое включение будет иметь место, если $h_{\alpha(s), m+1}^{i, 1}$ являются аналитическими коцепями. Проверка этого входит в проверку условий 1)-4), которую мы будем осушествлять последовательно с использованием индукции по $s$. Для всех $j=1, \ldots, j(m+1)-j(m)+2$ мы хотим построить компакты $L_{m+1, j}$ такие, что $L_{m} \subset L_{m+1,1} \subset \cdots \subset L_{m+1, j(m+1)-j(m)+2}$, и функции $\varphi_{\alpha(s), m+1}^{i}$, $s=1, \ldots, j(m)-2+j$, удовлетворяют условиям 1)-4), где в пп. 4, б), в) компакт $L_{m+1}$ нужно заменить на $L_{m+1, j}$, а условие 4 , а) заменить на неравенство

$$
H_{L_{m+1, j}}(z)+((n-j)(M+11)+n(M-m-1)(M+11)+10) \varepsilon|z|<H_{G}(z) .
$$

Если мы построим такую последовательность компактов, то теорема будет полностью доказана, поскольку в качестве компакта $L_{m+1}$ можно будет взять тогда $L_{m+1, j(m+1)-j(m)+2}$.

Напомним, что $L_{m+1,1}$ является замыканием множества $L+B(0, \varepsilon)$ (в частности, $\left.L \subset L_{m+1,1}\right)$. Поэтому согласно (3.18) компакт $L_{m+1,1}$ удовлетворяет (3.31). По построению $\varphi_{\alpha(s), m+1}^{i}, s=1, \ldots, j(m)-2$, совпадают с $\varphi_{\alpha(s), m}^{i}$. Следовательно, для указанных $s$ все наши условия выполнены в силу того, что по допушению индукции 1)-4) выполнены для $\varphi_{\alpha(s), m}^{i}$. Рассмотрим теперь $\varphi_{\alpha(j(m)-1), m+1}^{i}$. Они 
строились в соответствии с условиями 2, б)-г). Поэтому эти последние выполнены. Верно также 2, а), поскольку оно выполнено для $h_{\alpha(j(m)-1), m}^{i, 1}$, а по определению $h_{\alpha(j(m)-1), m+1}^{i, 1}=h_{\alpha(j(m)-1), m}^{i, 1}$. Проверим условие 1$)$. По построению с учетом условия 1) для $\varphi_{\alpha(j(m)-1), m}^{i}$ и условия 2, в) для $\varphi_{\alpha(j(m)-1), m+1}^{i}$ имеем

$$
\begin{gathered}
\sum_{i=k_{j(m)-2}(m+1)+1}^{n} \varphi_{\alpha(j(m)-1), m+1}^{i} F_{i}=\sum_{i=k_{j(m)-2}(m+1)+1}^{k_{j(m)-1}(m+1)-2} \varphi_{\alpha(j(m)-1), m}^{i} F_{i} \\
\quad+\left(\varphi_{\alpha(j(m)-1), m}^{k_{j(m)-1}(m+1)-1}+h_{\alpha(j(m)-1), m+1}^{k_{j(m)-1}(m+1)-1,3}\right) F_{k_{j(m)-1}(m+1)-1} \\
\quad+\sum_{i=k_{j(m)-1}(m+1)}^{n}\left(\varphi_{\alpha(j(m)-1), m}^{i}+\varphi_{\alpha(j(m)-1), m+1}^{i, k_{j(m)-1}(m+1)-1} F_{k_{j(m)-1}(m+1)-1}\right) F_{i} \\
=\sum_{i=k_{j(m)-2}(m)+1}^{n} \varphi_{\alpha(j(m)-1), m}^{i} F_{i}=\varphi_{\alpha(j(m)-1), m}^{k_{j(m)-2}(m)}=\varphi_{\alpha(j(m)-1), m+1}^{k_{j(m)-2}(m+1)} .
\end{gathered}
$$

Это дает условие 1). По построению $\varphi_{\alpha(j(m)-1), m+1}^{i}=\varphi_{\alpha(j(m)-1), m}^{i}$ для всех $i=$ $k_{j(m)-2}(m)+1=k_{j(m)-2}(m+1)+1, \ldots, k_{j(m)-1}(m)-1=k_{j(m)-1}(m+1)-2$. Следовательно, условие $3, \mathrm{a})$ верно для $\varphi_{\alpha(j(m)-1), m+1}^{i}, i=k_{j(m)-2}(m+1)+1$, $\ldots, k_{j(m)-1}(m+1)-2$. Поскольку

$$
\varphi_{\alpha(j(m)-1), m+1}^{i, k_{j(m)-1}(m+1)-1}=A_{\alpha(j(m)-1)}^{i}, \quad i=k_{j(m)-1}(m+1), \ldots, n
$$

то из $(3.28)$, условий 3, б $), 1)$ для $\varphi_{\alpha(j(m)-1), m}^{i}$ и условия 2, в $)$ для $\varphi_{\alpha(j(m)-1), m+1}^{i}$ получаем

$$
\begin{aligned}
\Delta \varphi_{\alpha(j(m)-1), m+1}^{k_{j(m)-1}(m+1)-1} & =\Delta \varphi_{\alpha(j(m)-1), m}^{k_{j(m)-1}(m+1)-1}+\Delta h_{\alpha(j(m)-1), m+1}^{k_{j(m)-1}(m+1)-1} \\
& =\Delta \varphi_{\alpha(j(m)-1), m}^{k_{j(m)-1}(m)} \sum_{i=k_{j(m)-1}(m+1)}^{n} \Delta \varphi_{\alpha(j(m)-1), m+1}^{i, k_{j(m)-1}(m+1)-1} F_{i} \\
& =\varphi_{\alpha(j(m)), m}^{k_{j(m)-1}(m)}-\sum_{i=k_{j(m)-1}(m)+1}^{n} \varphi_{\alpha(j(m)), m}^{i} F_{i} \equiv 0 .
\end{aligned}
$$

Таким образом, мы проверили условие 3, a). Условие 3, б) выполнено по построению.

Проверим 3,в). Прежде всего отметим следующее. Поскольку $k_{1}(m+1)=$ $k_{1}(m), \ldots, k_{j(m)-2}(m+1)=k_{j(m)-2}(m), k_{j(m)-1}(m+1)=k_{j(m)-1}(m)+1$, то все номера $t(m+1, j(m), p, r)$ из условия 3, в), за исключением одного (когда $p=$ $j(m)-1$ и $\left.r=k_{j(m)-1}(m+1)-1=k_{j(m)-1}(m)\right)$, совпадают с соответствующими номерами $t(m, j(m), p, r)$, т.е. $t(m+1, j(m), p, r)=t(m, j(m), p, r)$. Номер же $t\left(m+1, j(m), j(m)-1, k_{j(m)-1}(m+1)-1\right)$, как легко видеть, равен $m$. Поэтому 
для всех $i=k_{j(m)-1}(m+1), \ldots, n$ из определений $h_{\alpha(j(m)-1), m+1}^{i, j},(3.28)$ и условий 2, б), г), 3, в) для $\varphi_{\alpha(s), m}^{i}$ получаем

$$
\begin{aligned}
& \Delta h_{\alpha(j(m)-1), m+1}^{i, 2}+\Delta h_{\alpha(j(m)-1), m+1}^{i, 4}=\Delta h_{\alpha(j(m)-1), m}^{i, 2}+\Delta h_{\alpha(j(m)-1), m}^{i, 4} \\
& +\Delta \varphi_{\alpha(j(m)-1), m+1}^{i, k_{j(m)-1}(m)} F_{k_{j(m)-1}(m)} \\
& =-\sum_{p} \sum_{r=k_{p-1}(m)+1}^{k_{p}(m)-1}(-1)^{j(m)-p} \times \\
& \times\left[h_{\alpha(j(m)), t(m, j(m), p, r)}^{i, 1}+h_{\alpha(j(m)), t(m, j(m), p, r)}^{i, 3}\right] F_{r} \\
& -\sum_{p} \sum_{r=k_{p-1}(m)+1}^{k_{p}(m)-1}(-1)^{j(m)-p}\left[\sum_{j=k_{j(m)-1}(m)}^{i-1} \varphi_{\alpha(j(m)), t(m, j(m), p, r)}^{i, j} F_{j}\right] F_{r} \\
& +h_{\alpha(j(m)), m}^{i, 1} F_{k_{j(m)-1}(m)}+h_{\alpha(j(m)), m}^{i, 3} F_{k_{j(m)-1}(m)} \\
& +\sum_{j=k_{j(m)-1}(m)+1}^{i-1} \varphi_{\alpha(j(m)), m}^{i, j} F_{j} F_{k_{j(m)-1}(m)} \\
& +\sum_{p} \sum_{r=k_{p-1}(m)+1}^{k_{p}(m)-1}(-1)^{j(m)-p} \varphi_{\alpha(j(m)), t(m, j(m), p, r)}^{i, k_{j(m)-1}(m)} F_{r} F_{k_{j(m)-1}(m)} \\
& =-\sum_{p} \sum_{r=k_{p-1}(m+1)+1}^{k_{p}(m+1)-1}(-1)^{j(m)-p_{\times}} \\
& \times\left[h_{\alpha(j(m)), t(m+1, j(m), p, r)}^{i, 1}+h_{\alpha(j(m)), t(m+1, j(m), p, r)}^{i, 3}\right] F_{r} \\
& -\sum_{p} \sum_{r=k_{p-1}(m+1)+1}^{k_{p}(m+1)-1}(-1)^{j(m)-p} \times \\
& \times\left[\sum_{j=k_{j(m+1)-1}(m)}^{i-1} \varphi_{\alpha(j(m)), t(m+1, j(m), p, r)}^{i, j} F_{j}\right] F_{r} .
\end{aligned}
$$

Таким образом, условие 3, в) выполнено.

Остается проверить условие 4) с учетом указанных выше замен. Мы уже установили, что компакт $L_{m+1,1}$ удовлетворяет $(3.31)$. Условие 4, б) выполнено в силу допущения индукции, поскольку $h_{\alpha(j(m)-1), m+1}^{i, 1}=h_{\alpha(j(m)-1), m}^{i, 1}$ и $L_{m} \subset L_{m+1,1}$. Для функций $\varphi_{\alpha(j(m)-1), m+1}^{i, k_{j(m)-1}(m+1)-1}$ условие 4, в) вьполнено в силу $(3.27)$, так как $H_{j(m), m}=H_{j(m)-1, m}-H_{K_{j}}=H_{j(m)-1, m+1}-H_{K_{j}}$, где $j=k_{j(m)-1}(m)$. Для всех остальных функций $\varphi_{\alpha(j(m)-1), m+1}^{i, r}$ условие 4,в), как и 4,б), выполнено по допущению индукции.

Итак, мы построили компакт $L_{m+1,1}$ и проверили условия 1)-4) (с указанными вьше заменами) для функций $\varphi_{\alpha(s), m+1}^{i}, s=1, \ldots, j(m)-1$. Предположим, что мы уже построили компакты $L_{m+1,1}, \ldots, L_{m+1, j}$ и проверили все необходимые 
условия. Рассмотрим теперь функции $\varphi_{\alpha(s), m+1}^{i}$, где $s=j(m)-2+j+1$. Прежде всего отметим, что функции $h_{\alpha(s), m+1}^{i}$ строились вполне аналогично функциям $h_{\alpha(s), 1}^{i}=\varphi_{\alpha(s), 1}^{i}$. Поэтому точно так же, как и для $h_{\alpha(s), 1}^{i}$, доказывается, что $h_{\alpha(s), m+1}^{i}$ являются аналитическими коцепями и удовлетворяют условию 2 , а). Кроме того, как и в случае $m=1$, с использованием лемм 3.2 и 3.5 по компакту $L_{m+1, j}$ строится компакт $L_{m+1, j+1}$ такой, что выполнены (3.31) и условие 4, б), где $L_{m+1}$ заменен на $L_{m+1, j+1}$. Заметим также, что условие 4, в) в данном случае отсутствует, так как функций $\varphi_{\alpha(s), m+1}^{i, r}$ не существует, а поскольку $k_{s}(m+1)=k_{s-1}(m+1)+1$, то по построению выполнены условия 2, б), в) и 3, a). Наконец, по построению же выполнены условия 2, г) и 3, б).

Остается проверить условия 1) и 3, в). Проверим вначале 1 ). Согласно условиям 1) и 3, a), б), выполненным по допущению индукции для $\varphi_{\alpha(k), m+1}^{i}, k<s$, с учетом равенства $\Delta^{2}=0$ получаем

$$
\begin{aligned}
0 & =\Delta^{2} \varphi_{\alpha(s-2), m+1}^{k_{s-2}(m+1)}=\Delta \varphi_{\alpha(s-1), m+1}^{k_{s-2}(m+1)}=\sum_{i=k_{s-2}(m+1)+1}^{n} \Delta \varphi_{\alpha(s-1), m+1}^{i} F_{i} \\
& =\sum_{i=k_{s-1}(m+1)}^{n} \Delta \varphi_{\alpha(s-1), m+1}^{i} F_{i} \\
& =\varphi_{\alpha(s), m+1}^{k_{s-1}(m+1)} F_{k_{s-1}(m+1)}+\sum_{i=k_{s-1}(m+1)+1}^{n} \Delta \varphi_{\alpha(s-1), m+1}^{i} F_{i} .
\end{aligned}
$$

Таким образом,

$$
\varphi_{\alpha(s), m+1}^{k_{s-1}(m+1)} F_{k_{s-1}(m+1)}=-\sum_{i=k_{s-1}(m+1)+1}^{n} \Delta \varphi_{\alpha(s-1), m+1}^{i} F_{i} .
$$

С другой стороны, согласно 2, в) $h_{\alpha(s-1), m+1}^{k_{s-1}(m+1), 3} \equiv 0$. Поэтому в силу 3, в) имеем

$$
\begin{aligned}
\varphi_{\alpha(s), m+1}^{k_{s-1}(m+1)}= & \Delta \varphi_{\alpha(s-1), m+1}^{k_{s-1}(m+1)}=\Delta h_{\alpha(s-1), m+1}^{k_{s-1}(m+1), 1}+\Delta h_{\alpha(s-1), m+1}^{k_{s-1}(m+1), 2}+\Delta h_{\alpha(s-1), m+1}^{k_{s-1}(m+1), 4} \\
= & \Delta h_{\alpha(s-1), m+1}^{k_{s-1}(m+1), 1}-\sum_{p} \sum_{r=k_{p-1}(m+1)+1}^{k_{p}(m+1)-1}(-1)^{s-p} \\
& \times\left[h_{\alpha(s), t(m+1, s, p, r)}^{k_{s-1}(m+1), 1}+h_{\alpha(s), t(m+1, s, p, r)}^{k_{s-1}(m+1), 3} F_{r} .\right.
\end{aligned}
$$

Отсюда с учетом условия 2, в) для $\varphi_{\alpha(s), t(m+1, s, p, r)}^{i}$, условия 2, г) для $\varphi_{\alpha(s), m+1}^{i}$ и равенств $h_{\alpha(s), m+1}^{i, 2} \equiv h_{\alpha(s), m+1}^{i, 3} \equiv 0$ получаем

$$
\begin{aligned}
\varphi_{\alpha(s), m+1}^{k_{s-1}(m+1)}= & \Delta h_{\alpha(s-1), m+1}^{k_{s-1}(m+1), 1}-\sum_{p} \sum_{r=k_{p-1}(m+1)+1}^{k_{p}(m+1)-1}(-1)^{s-p}\left[h_{\alpha(s), t(m+1, s, p, r)}^{k_{s-1}(m+1), 1}\right] F_{r} \\
& +\sum_{i=k_{s-1}(m+1)+1}^{n} h_{\alpha(s), m+1}^{i, 4} F_{i} \\
= & \Delta h_{\alpha(s-1), m+1}^{k_{s-1}(m+1), 1}-\sum_{p} \sum_{r=k_{p-1}(m+1)+1}^{k_{p}(m+1)-1}(-1)^{s-p}\left[h_{\alpha(s), t(m+1, s, p, r)}^{k_{s-1}(m+1), 1}\right] F_{r}
\end{aligned}
$$


114

$$
+\sum_{i=k_{s-1}(m+1)+1}^{n} \varphi_{\alpha(s), m+1}^{i} F_{i}-\sum_{i=k_{s-1}(m+1)+1}^{n} h_{\alpha(s), m+1}^{i, 1} F_{i} .
$$

Из этих соотношений следует, что для доказательства 1) достаточно установить равенство

$$
\begin{aligned}
& \Delta h_{\alpha(s-1), m+1}^{k_{s-1}(m+1), 1}-\sum_{p} \sum_{r=k_{p-1}(m+1)+1}^{k_{p}(m+1)-1}(-1)^{s-p}\left[h_{\alpha(s), t(m+1, s, p, r)}^{k_{s-1}(m+1), 1}\right] F_{r} \\
& =\sum_{i=k_{s-1}(m+1)+1}^{n} h_{\alpha(s), m+1}^{i, 1} F_{i} .
\end{aligned}
$$

Если левая часть этого равенства тождественно равна нулю, то по построению $h_{\alpha(s), m+1}^{i, 1} \equiv 0, i=k_{s-1}(m+1)+1, \ldots, n$. Следовательно, в этом случае равенство имеет место. Если же левая часть не является тождественным нулем, то согласно определению $h_{\alpha(s), m+1}^{i, 1}$ нужно установить равенство

$$
\begin{aligned}
& \quad \sum_{i=k_{s-1}(m+1)+1}^{n}\left[-\Delta h_{\alpha(s-1), m+1}^{i, 1}\right. \\
& \left.\quad+\sum_{p} \sum_{r=k_{p-1}(m+1)+1}^{k_{p}(m+1)-1}(-1)^{s-p} h_{\alpha(s), t(m+1, s, p, r)}^{i, 1} F_{r}\right] F_{k_{s-1}(m+1)}^{-1} F_{i} \\
& =\Delta h_{\alpha(s-1), m+1}^{k_{s-1}(m+1), 1}-\sum_{p} \sum_{r=k_{p-1}(m+1)+1}^{k_{p}(m+1)-1}(-1)^{s-p}\left[h_{\alpha(s), t(m+1, s, p, r)}^{k_{s-1}(m+1), 1}\right] F_{r} .
\end{aligned}
$$

По условию 2, в $) h_{\alpha(s-1), m+1}^{i, 3} \equiv 0, i=k_{s-1}(m+1)+1, \ldots, n$. Поэтому в силу (3.32) и условия 3, в) для $\varphi_{\alpha(s-1), m+1}^{i}, i=k_{s-1}(m+1)+1, \ldots, n$, получаем

$$
\begin{aligned}
& \varphi_{\alpha(s), m+1}^{k_{s-1}(m+1)} F_{k_{s-1}(m+1)}=-\sum_{i=k_{s-1}(m+1)+1}^{n}\left(\Delta h_{\alpha(s-1), m+1}^{i, 1}\right. \\
& \left.+\Delta h_{\alpha(s-1), m+1}^{i, 2}+\Delta h_{\alpha(s-1), m+1}^{i, 4}\right) F_{i} \\
& =-\sum_{i=k_{s-1}(m+1)+1}^{n}\left(\Delta h_{\alpha(s-1), m+1}^{i, 1}\right. \\
& \left.\quad-\sum_{p} \sum_{r=k_{p-1}(m+1)+1}^{k_{p}(m+1)-1}(-1)^{s-p} h_{\alpha(s), t(m+1, s, p, r)}^{i, 1} F_{r}\right) F_{i} \\
& +\sum_{i=k_{s-1}(m+1)+1}^{n} \sum_{p}^{k_{p}(m+1)-1} \sum_{\substack{r=k_{p-1}(m+1)+1 \\
i, k_{s-1}(m+1)}}(-1)^{s-p} \times \\
& \times \varphi_{\alpha(s), t(m+1, s, p, r)} F_{k_{s-1}(m+1)} F_{r} F_{i}
\end{aligned}
$$




$$
\begin{aligned}
& +\sum_{i=k_{s-1}(m+1)+2} \sum_{p} \sum_{r=k_{p-1}(m+1)+1}^{k_{p}(m+1)-1}(-1)^{s-p} \times \\
& \quad \times \sum_{j=k_{s-1}(m+1)+1}^{i-1} \varphi_{\alpha(s), t(m+1, s, p, r)}^{i, j} F_{j} F_{r} F_{i} \\
& +\sum_{i=k_{s-1}(m+1)+1}^{n} \sum_{p} \sum_{r=k_{p-1}(m+1)+1}^{k_{p}(m+1)-1}(-1)^{s-p} h_{\alpha(s), t(m+1, s, p, r)}^{i, 3} F_{r} F_{i} .
\end{aligned}
$$

Здесь мы использовали разделение суммы по $j$ на две части $\left(j=k_{s-1}(m+1)\right.$ и $j=$ $\left.k_{s-1}(m+1)+1, \ldots, i-1\right)$. При этом суммирование по $i$ в предпоследнем слагаемом ведется с $k_{s-1}(m+1)+2\left(\right.$ а не с $\left.k_{s-1}(m+1)+1\right)$, поскольку при $i=k_{s-1}(m+1)+1$ для каждого $p$ и $r$ сушествует лишш одна функция $\varphi_{\alpha(s), t(m+1, s, p, r)}^{k_{s-1}(m+1)+1, k_{s-1}(m+1)} \cdot$ Из условия 2,в) следует, что сумма трех последних строк равна нулю. Кроме того, по условию $2, \Gamma)$ для $\varphi_{\alpha(s), m+1}^{i}$ пятая строка представляет собой сумму функций $h_{\alpha(s), m+1}^{i, 4} F_{i} F_{k_{s-1}(m+1)}$ по всем $i=k_{s-1}(m+1)+1, \ldots, n$. Учитьвая сказанное и сравнивая (3.33) и (3.35), легко получаем (3.34). Таким образом, равенство из условия 1) имеет место.

Осталось проверить 3, в). По построению $h_{\alpha(s), m+1}^{i, 2} \equiv 0, i=k_{s-1}(m+1)+1=$ $k_{s}(m+1), \ldots, n$. Поэтому из условий $\left.2, г\right)$ и 2, б) получаем

$$
\begin{aligned}
& \Delta h_{\alpha(s), m+1}^{i, 2}+\Delta h_{\alpha(s), m+1}^{i, 4}=\Delta h_{\alpha(s), m+1}^{i, 4} \\
& \quad=\sum_{p} \sum_{r=k_{p-1}(m+1)+1}^{k_{p}(m+1)-1}(-1)^{s-p} \Delta \varphi_{\alpha(s), t(m+1, s, p, r)}^{i, k_{s-1}(m+1)} F_{r} \\
& =\sum_{p} \sum_{r=k_{p-1}(m+1)+1}^{k_{p}(m+1)-1}(-1)^{s-p} \varphi_{\alpha(s+1), \tau(p, r)}^{i} F_{r} \\
& =-\sum_{p}^{k_{p}(m+1)-1} \sum_{r=k_{p-1}(m+1)+1}(-1)^{s+1-p} \varphi_{\alpha(s+1), \tau(p, r)}^{i} F_{r} .
\end{aligned}
$$

Через $\tau(p, r)$ мы обозначили здесь номер $l(k, s, q)$ из условия $2, б)$ такой, что $k=$ $t(m+1, s, p, r)$ и $q=k_{s-1}(m+1)$. Согласно $\left.2, \Gamma\right)$ и $\left.2, б\right) \tau(p, r)$ однозначно определяется из равенств:

$$
\begin{gathered}
j(\tau(p, r))=s+1, \\
k_{1}(\tau(p, r))=k_{1}(m+1), \ldots, k_{p-1}(\tau(p, r))=k_{p-1}(m+1), \quad k_{p}(\tau(p, r))=r, \\
k_{p+1}(\tau(p, r))=k_{p}(m+1), \ldots, k_{s}(\tau(p, r))=k_{s-1}(m+1) .
\end{gathered}
$$

Эти равенства в точности совпадают с равенствами, согласно которым определяется номер $t(m+1, s+1, p, r)$ в условии $2, г)$. Поэтому из предыдущего с учетом 
условия 2, б) и того, что $k_{s}(\tau(p, r))+1=k_{s-1}(m+1)+1=k_{s}(m+1)$, получаем

$$
\begin{aligned}
& \Delta h_{\alpha(s), m+1}^{i, 2}+\Delta h_{\alpha(s), m+1}^{i, 4}= \\
& =-\sum_{p} \sum_{r=k_{p-1}(m+1)+1}^{k_{p}(m+1)-1}(-1)^{s+1-p} \sum_{j=1}^{4} h_{\alpha(s+1), t(m+1, s+1, p, r)}^{i, j} F_{r} \\
& =-\sum_{p} \sum_{r=k_{p-1}(m+1)+1}^{k_{p}(m+1)-1}(-1)^{s+1-p_{\times}} \\
& \times\left[h_{\alpha(s+1), t(m+1, s+1, p, r)}^{i, 1}+h_{\alpha(s+1), t(m+1, s+1, p, r)}^{i, 3}\right] F_{r} \\
& -\sum_{p} \sum_{r=k_{p-1}(m+1)+1}^{k_{p}(m+1)-1}(-1)^{s+1-p} \sum_{j=k_{s}(m+1)}^{i-1} \varphi_{\alpha(s+1), t(m+1, s+1, p, r)}^{i, j} F_{j} F_{r} \\
& -\sum_{p} \sum_{r=k_{p-1}(m+1)+1}^{k_{p}(m+1)-1}(-1)^{s+1-p} h_{\alpha(s+1), t(m+1, s+1, p, r)}^{i, 4} F_{r} .
\end{aligned}
$$

Таким образом, 3,в) будет доказано, если верно равенство

$$
\sum_{p} \sum_{r=k_{p-1}(m+1)+1}^{k_{p}(m+1)-1}(-1)^{s+1-p} h_{\alpha(s+1), t(m+1, s+1, p, r)}^{i, 4} F_{r} \equiv 0 .
$$

По условию 2, г) с учетом равенства $k_{s}(t(m+1, s+1, p, r))=k_{s-1}(m+1)$ имеем

$$
h_{\alpha(s+1), t(m+1, s+1, p, r)}^{i, 4}=\sum_{q} \sum_{\nu=k_{q-1}(m)+1}^{k_{q}(m)-1}(-1)^{s+1-q} \varphi_{\alpha(s+1), \gamma(p, r, q, \nu)}^{i, k_{s-1}(m+1)} F_{\nu}
$$

где $\gamma(p, r, q, \nu)$ обозначает номер $t(k, s+1, q, \nu)$ из условия $2, г)$ такой, что $k=$ $t(m+1, s+1, p, r)$. Нетрудно видеть, что $\gamma(p, r, q, \nu)=\gamma(q-1, \nu, p, r)$, если $p<q$, и $\gamma(p, r, q, \nu)=\gamma(q, \nu, p+1, r)$, если $p \geqslant q$. Но тогда

$$
\begin{aligned}
& (-1)^{s+1-p+s+1-q} \varphi_{\alpha(s+1), \gamma(p, r, q, \nu)}^{i, k_{s-1}(m+1)} F_{r} F_{\nu} \\
& \quad+(-1)^{s+1-q+1+s+1-p} \varphi_{\alpha(s+1), \gamma(q-1, \nu, p, r)}^{i, k_{s-1}(m+1)} F_{\nu} F_{r} \equiv 0 .
\end{aligned}
$$

Аналогичное тождество верно и для номеров $\gamma(p, r, q, \nu)$ и $\gamma(q, \nu, p+1, r)$. Таким образом, (3.36) выполнено, и теорема полностью доказана.

В качестве примера на применение теоремы 3.7 рассмотрим систему линейных дифференциальных уравнений конечного порядка с постоянными коэффициентами. Эта система является частным случаем системы (1.1), который возникает в ситуации, когда функционалы $\mu_{i}, i=1, \ldots, n$, представляют собой линейные комбинации $\delta$-функции и ее производных. Преобразованиями Лапласа таких функционалов являются многочлены. Они имеют минимальный экспоненциальный тип, т.е. их сопряженные диаграммы $K_{i}$ совпадают с началом координат. Тогда области $G_{i}$ совпадают с $D$. Как отмечалось вьше, любая система многочленов имеет регулярньй рост (и, следовательно, слабый регулярный рост) всюду в плоскости. Таким образом, имеет место 
СлЕДСТВИЕ 3.8. Пусть система (1.1) является системой линейных дифференциальных уравнений конечного порядка с постоянными коэффичиентами. Тогда она разрешима в $H(D)$ при любой допустимой правой части из $H^{n}(D)$.

\section{§4. Необходимые условия разрешимости}

В этом параграфе мы приведем некоторые необходимые условия разрешимости системы (1.1). В частности, покажем, что условия теоремы 3.7 дают критерий разрешимости для выпуклых областей с гладкой гранищей.

Прежде всего сделаем некоторые замечания, касаюшиеся гранищы выпуклого множества $T \subset \mathbb{C}$. Пусть $z$ - точка относительной границы $\partial T$. Выпуклое множество на плоскости, отличное от точки, может лежать на некоторой прямой, и тогда является интервалом этой прямой, либо представляет собой выпуклую область плюс некоторое подмножество ее границы. В первом случае границей являются коншы интервала (если они есть), а во втором относительная граница совпадает с обычной границей области. Введем множество

$$
\Gamma(z)=\left\{\eta: H_{T}(\eta)=\operatorname{Re} z \eta\right\} .
$$

Оно непустое. Действительно, известно, что через каждую граничную точку $z$ выпуклого множества проходит хотя бы одна опорная прямая. Если вектор $\bar{\eta}$ перпендикулярен этой прямой, то $\eta \in \Gamma(z)$. Множество $\Gamma(z)$ является конусом в силу того, что функция $H_{T}(\eta)$ положительно однородна порядка один. Пусть $\eta, \zeta \in \Gamma(z)$. Тогда в силу выпуклости $H_{T}(\eta)$ имеем

$$
\begin{aligned}
& H_{T}(\tau \eta+(1-\tau) \zeta)-\operatorname{Re}(\tau \eta+(1-\tau) \zeta) z \\
& \quad \leqslant \tau\left(H_{T}(\eta)-\operatorname{Re} z \eta\right)+(1-\tau)\left(H_{T}(\zeta)-\operatorname{Re} z \zeta\right)=0, \quad \tau \in[0,1] .
\end{aligned}
$$

$\mathrm{C}$ другой стороны, из определения опорной функции следует, что $\operatorname{Re} z \xi \leqslant H_{T}(\xi)$ $\forall \xi \in \mathbb{C}$. Поэтому верно равенство $\operatorname{Re} z \xi=H_{T}(\xi), \xi=\tau \eta+(1-\tau) \zeta, \tau \in[0,1]$. Таким образом, $\Gamma(z)$ является выпуклым конусом. Возможны следующие ситуации: I) $Г(z)$ - луч; II) $Г(z)$ - угол раствора не больше $\pi$; III) $\Gamma(z)$ - вся плоскость. Единственная оставшаяся возможность, когда $\Gamma(z)$ - прямая, не реализуется. Если множество $\Gamma(z)$ содержит прямую, то оно является либо полуплоскостью, либо плоскостью. В случае I) множество $T$ представляет собой область вместе с некоторым подмножеством своей границы. Если $\Gamma(z)$ - полуплоскость, то $T$ лежит на прямой. В случае III) $T$ состоит лишь из точки $z$. Отметим еше, что для каждого $\eta \in \mathbb{C} \backslash \bar{I}_{T}$ опорная прямая, перпендикулярная вектору $\bar{\eta}$, пересекает $\partial T$. В случае неограниченного множества возможна ситуация, когда для одной или обеих точек $\eta \in \partial I_{T} \backslash I_{T}$ опорная прямая, перпендикулярная вектору $\bar{\eta}$, не пересекает $\partial T$, а является асимптотой для граничной кривой.

Через $\Gamma(T)$ обозначим замыкание объединения всех множеств $\Gamma(z), z \in \partial T$, которые являются лучами. Г( $z)$ будет лучом, если $z$-гладкая точка границы, т.е. через нее проходит только одна опорная прямая. В частности, если $T$ - область с гладкой $\left(C^{1}\right)$ границей, то $\Gamma(z)$ - луч для любого $z \in \partial T$. В этом случае имеет место равенство $\Gamma(T)=\mathbb{C} \backslash$ int $I_{T}$. В связи с этим $\Gamma(T)$ можно назвать множеством гладких направлений множества $T$. 
В работах [3] и [4] уже рассматривались множества (обозначаемые там $\mathbf{P}_{D}^{*}$ ), которые, подобно $\Gamma(T)$, строятся по конусам $\Gamma(z)$. Причем множества эти вводились для областей $D$ в многомерном комплексном пространстве. Для таких областей мы можем также ввести множество $\Gamma(D)$. Сравнивая определения $\Gamma(D)$ здесь и $\mathbf{P}_{D}^{*}$ в [4], легко убедиться в том, что имеет место включение $\Gamma(D) \subset \mathbf{P}_{D}^{*}$, которое, вообше говоря, является строгим. Однако для плоских областей множества $\Gamma(D)$ и $\mathbf{P}_{D}^{*}$ совпадают. Это совпадение позволяет нам воспользоваться здесь одним из результатов работы [4], касающихся множества $\mathbf{P}_{D}^{*}$. Точнее говоря, нам понадобится теорема 8.2 этой работы, которую мы сейчас сформулируем для случая плоских областей.

Лемма 4.1. Пусть $G$ - выпуклая область в $\mathbb{C}, z_{0} \in \partial G$ такова, что $\Gamma\left(z_{0}\right)-$ луч, $\left\{\eta_{0}\right\}=\mathbb{S} \cap \Gamma\left(z_{0}\right) u \varepsilon^{\prime}, \delta^{\prime}>0$. Найдется $\eta^{*} \in B\left(\eta_{0}, \delta^{\prime}\right)$ такое, что для любой последовательности $\left\{t_{k}\right\}_{k=1}^{\infty}, \quad 1 \leqslant t_{k} \leqslant 2^{-1} t_{k+1}, \quad k=1,2, \ldots$, любых пололительных $\delta \leqslant \delta_{0}\left(\varepsilon^{\prime}\right)$ и $\varepsilon \leqslant \varepsilon_{0}\left(\delta, \varepsilon^{\prime}\right)$ существует целая функиия $\varphi(\eta)$ әкспоненциального типа, удовлетворяющая следующим условиям:

1) $h_{\varphi}(\eta) \leqslant H_{G}(\eta), \eta \in \mathbb{C}$

2) существует $\eta_{0}^{*} \in B\left(\eta^{*}, \delta\right) \cap \mathbb{S}$ такое, ито $h_{\varphi}\left(\eta_{0}^{*}\right)=H_{G}\left(\eta_{0}^{*}\right)$;

3) $h_{\varphi}(\eta) \leqslant H_{G}(\eta)-\varepsilon, \eta \in \mathbb{S} \backslash B\left(\eta^{*}, \delta\right)$;

4) существует $R_{0}>0$ такое, что

$\frac{\ln |\varphi(t \eta)|}{t} \leqslant H_{G}(\eta)-\varepsilon, \quad \eta \in \mathbb{S} \cap \overline{B\left(\eta^{*}, \delta\right)}, \quad t \notin \bigcup_{k=1}^{\infty}\left[(1-\delta) t_{k},(1+\delta) t_{k}\right] \cup\left[0, R_{0}\right] ;$

5) существует $R_{1}>0$ такое, что

$$
\frac{\ln |\varphi(t \eta)|}{t} \leqslant H_{G}\left(\eta_{0}\right)+\varepsilon^{\prime}, \quad \eta \in \mathbb{S} \cap \overline{B\left(\eta^{*}, \delta\right)}, \quad t \geqslant R_{1}
$$

Нам также понадобится предложение 9.3 из работы [4]. Чтобы его сфформулировать, нужно вначале ввести понятие нижнего индикатора.

Пусть $\psi(z)$ - субгармоническая функция порядка не выше $\rho>0$ и конечного типа при порядке $\rho$. Нижним индикатором $\psi(z)$ (см. [11], [12], [18]) называется функция

$$
\underline{h}_{\psi}(z)=\lim _{\delta \rightarrow 0} \underset{t \rightarrow+\infty}{\lim _{t \rightarrow+\infty}} \frac{1}{\pi \delta^{2}} \int_{B(z, \delta)} \frac{\psi(t y)}{t^{\rho}} d \sigma .
$$

Всегда выполнено неравенство

$$
\underline{h}_{\psi}(z) \leqslant h_{\psi}(z) \quad \forall z \in \mathbb{C},
$$

где $h_{\psi}$ - индикатор $\psi(z)$, который определяется так же, как и для функции $\psi=$ $\ln |f|$ ( $f$ целая). Используя нижний индикатор, можно дать следующее необходимое и достаточное условие регулярности роста. Функция $\psi(z)$ имеет регулярный pocm на луче $t z, t>0$, тогда и только тогда, когда $\underline{h}_{\psi}(z)=h_{\psi}(z)$. 
ЛЕмма 4.2 [4]. Пусть $\psi(z)$ - субгармоническая функиия порядка не выше $\rho>0$ и конечного типа при порядке $\rho, z_{0} \in \mathbb{S}$. Eсли $\underline{h}_{\psi}\left(z_{0}\right)<C_{0}$, то существуют $\delta_{0}>0$ и возрастающая $(\kappa+\infty)$ последовательность $\left\{t_{k}\right\}$ такие, что

$$
\frac{\psi\left(t_{k} z\right)}{t_{k}^{\rho}} \leqslant C_{0} \quad \forall z \in B\left(z_{0}, \delta_{0}\right), \quad k=1,2, \ldots
$$

Пусть функции $f_{i}$, компакты $K_{i}$ и области $G_{i}, D$ - те же, что и в предыдущих параграфах. Положим

$$
\psi(z)=\max _{1 \leqslant i \leqslant n}\left[\ln \left|f_{i}(z)\right|+\sum_{j=1, j \neq i}^{n} H_{K_{j}}(z)\right] .
$$

Функция $\psi(z)$ является субгармонической порядка не вьше один и конечного типа при порядке один. Нетрудно видеть, что ее индикатор вычисляется по формуле (напомним, что $h_{f_{i}} \equiv H_{K_{i}}$ )

$$
h_{\psi}(z)=\sum_{j=1}^{n} H_{K_{j}}(z)
$$

ПРЕДЛОЖЕНИЕ 4.3. Если система (1.1) разрешима в $H(D)$ для любой допустимой правой части из $H\left(G_{1}\right) \times \cdots \times H\left(G_{n}\right)$, то функция $\psi(z)$ имеет регулярный рост на каждом луче из множества $\Gamma(G)$.

ДокАЗАТЕЛЬСТво. Пусть $\eta^{\prime} \in \mathbb{S} \cap \Gamma(G)$. Предположим, что $\psi(z)$ не имеет регулярного роста на луче $t \eta^{\prime}$. Тогда в силу сказанного вьше $\underline{h}_{\psi}\left(\eta^{\prime}\right)<h_{\psi}\left(\eta^{\prime}\right)$. Выберем $\varepsilon^{\prime}>0$ так, что $\underline{h}_{\psi}\left(\eta^{\prime}\right)+6 \varepsilon^{\prime}<h_{\psi}\left(\eta^{\prime}\right)$. По лемме 4.2 найдем последовательность $\left\{t_{k}\right\}$ и число $\delta_{0} \in(0,1 / 3)$ такие, что

$$
\frac{\psi\left(t_{k} \eta\right)}{t_{k}} \leqslant h_{\psi}\left(\eta^{\prime}\right)-5 \varepsilon^{\prime}=\sum_{j=1}^{n} H_{K_{j}}\left(\eta^{\prime}\right)-5 \varepsilon^{\prime}, \quad \eta \in B\left(\eta^{\prime}, 2 \delta_{0}\right), \quad k=1,2, \ldots
$$

Уменьшая при необходимости $\delta_{0}>0$, как и в $(3.3)$, получаем

$$
\sum_{j=1}^{n}\left|H_{K_{j}}(\eta)-H_{K_{j}}(\xi)\right|<\varepsilon^{\prime} \quad \forall \eta \in B\left(\xi, 2 \delta_{0}\right), \quad \forall \xi \in \mathbb{S} .
$$

Переходя к подпоследовательности, можно считать, что $2 t_{k} \leqslant t_{k+1}, k=1,2, \ldots$ Согласно определению множества $\Gamma(G)$ существуют $\eta_{0} \in \mathbb{S} \cap B\left(\eta^{\prime}, \delta_{0}\right)$ и $z_{0} \in$ $\partial G$ такие, что $\eta_{0} \in \Gamma\left(z_{0}\right)$ и $\Gamma\left(z_{0}\right)-$ луч. Из двух последних неравенств с учетом выбора $\delta_{0}$ получаем

$$
\begin{aligned}
\psi\left(t_{k} \eta\right) & \leqslant \sum_{j=1}^{n} H_{K_{j}}\left(t_{k} \eta\right)-4 \varepsilon^{\prime} t_{k} \leqslant \sum_{j=1}^{n} H_{K_{j}}\left(t_{k} \eta\right)-3 \varepsilon^{\prime}\left|t_{k} \eta\right|+\varepsilon^{\prime} t_{k}\left(3\left(1+\delta_{0}\right)-4\right) \\
& \leqslant \sum_{j=1}^{n} H_{K_{j}}\left(t_{k} \eta\right)-3 \varepsilon^{\prime}\left|t_{k} \eta\right|, \quad \eta \in B\left(\eta_{0}, \delta_{0}\right), \quad k=1,2, \ldots
\end{aligned}
$$


В силу полунепрерывности снизу функции $H_{G}(\eta)$ найдется $\delta^{\prime} \in(0, \delta / 2)$ такое, что

$$
H_{G}\left(\eta_{0}\right) \leqslant H_{G}(\eta)+\varepsilon^{\prime} \quad \forall \eta \in B\left(\eta_{0}, 2 \delta^{\prime}\right) .
$$

Переходя к подпоследовательности, можно считать, что $1 \leqslant t_{k} \leqslant 2^{-1} t_{k+1}$, $k=1,2, \ldots$ Пусть $\varphi(\eta)$ - функция, существование которой утверждается в лемме 4.1. При этом на число $\delta$, фигурирующее в условиях 1)-5) этой леммы, наложим дополнительное ограничение: $\delta<\delta^{\prime} / 3$. Тогда для любых $t \in\left[(1-\delta) t_{k},(1+\delta) t_{k}\right]$, $k=1,2, \ldots$, и $\eta \in \mathbb{S} \cap B\left(\eta^{*}, \delta\right)$ точка $t \eta$ принадлежит кругу $B\left(\eta^{*}, \delta^{\prime}\right)$, который, в свою очередь, лежит в круге $B\left(\eta_{0}, \delta_{0}\right)$. Согласно свойству 2$)$ леммы 4.1 и определению индикатора найдем последовательность $\left\{r_{m}\right\}_{m=1}^{\infty}$ такую, что $r_{m} \rightarrow+\infty$ и

$$
\lim _{m \rightarrow \infty} \frac{\ln \left|\varphi\left(r_{m} \eta_{0}^{*}\right)\right|}{r_{m}}=H_{G}\left(\eta_{0}^{*}\right) .
$$

Поскольку $\psi(\eta)$ представляет собой максимум конечного числа функций, то найдутся номер $i=1, \ldots, n$ и подпоследовательность $\left\{r_{m_{j}}\right\}_{j=1}^{\infty}$ такие, что

$$
\psi\left(r_{m_{j}} \eta_{0}^{*}\right)=\ln \left|f_{i}\left(r_{m_{j}} \eta_{0}^{*}\right)\right|+\sum_{p=1, p \neq i}^{n} H_{K_{p}}\left(r_{m_{j}} \eta_{0}^{*}\right), \quad j=1,2, \ldots
$$

В силу ограниченности множеств $K_{p}$ для каждого $p=1, \ldots, n$ найдется точка $z_{p} \in \partial K_{p} \subset K_{p}$ такая, что $\eta_{0}^{*}$ принадлежит $\Gamma\left(z_{p}\right)$. Рассмотрим функцию

$$
F(\eta)=\varphi(\eta) f_{i}(\eta) \prod_{p=1, p \neq i}^{n} \exp \left[z_{p} \eta\right]
$$

Покажем, что $F \in I(D, \Lambda)$. Так как в определении $F$ участвует функция $f_{i}$, то достаточно установить включение $F \in \mathbf{P}_{D}$. С учетом свойства 3$)$ леммы 4.1 получаем

$$
\begin{aligned}
h_{F}(\eta) & \leqslant h_{\varphi}(\eta)+h_{f_{i}}(\eta)+\sum_{p=1, p \neq i}^{n} \operatorname{Re}\left[z_{p} \eta\right] \leqslant h_{\varphi}(\eta)+\sum_{p=1}^{n} H_{K_{p}}(\eta) \\
& \leqslant H_{G}(\eta)-\varepsilon+\sum_{p=1}^{n} H_{K_{p}}(\eta)=H_{D}(\eta)-\varepsilon \quad \forall \eta \in \mathbb{S} \backslash B\left(\eta^{*}, \delta\right) .
\end{aligned}
$$

Точно так же из свойства 4) функции $\varphi(\eta)$ следует, что

$$
\begin{aligned}
& \frac{\ln |F(t \eta)|}{t}=\frac{\ln |\varphi(t \eta)|}{t}+\frac{\ln \left|f_{i}(t \eta)\right|}{t}+\sum_{p=1, p \neq i}^{n} \operatorname{Re}\left[z_{p} \eta\right] \\
& \leqslant H_{G}(\eta)-\varepsilon+\frac{\ln \left|f_{i}(t \eta)\right|}{t}+\sum_{p=1, p \neq i}^{n} H_{K_{p}}(\eta), \\
& \eta \in \mathbb{S} \cap \overline{B\left(\eta^{*}, \delta\right)}, \quad t \notin \bigcup_{k=1}^{\infty}\left[(1-\delta) t_{k},(1+\delta) t_{k}\right] \cup\left[0, R_{0}\right] .
\end{aligned}
$$


Наконец, согласно (4.1), (4.2) и свойству 5) функции $\varphi(\eta)$ имеем

$$
\begin{aligned}
\frac{\ln |F(t \eta)|}{t} & =\frac{\ln |\varphi(t \eta)|}{t}+\frac{\ln \left|f_{i}(t \eta)\right|}{t}+\sum_{p=1, p \neq i}^{n} \operatorname{Re}\left[z_{p} \eta\right] \\
& \leqslant H_{G}(\eta)+2 \varepsilon^{\prime}+\frac{\ln \left|f_{i}(t \eta)\right|}{t}+\sum_{p=1, p \neq i}^{n} H_{K_{p}}(\eta) \\
& \leqslant H_{G}(\eta)+2 \varepsilon^{\prime}+\sum_{p=1}^{n} H_{K_{p}}(\eta)-3 \varepsilon^{\prime}=H_{D}(\eta)-\varepsilon^{\prime}, \\
& \eta \in \mathbb{S} \cap \overline{B\left(\eta^{*}, \delta\right)}, \quad t \in \bigcup_{k=1}^{\infty}\left[(1-\delta) t_{k},(1+\delta) t_{k}\right] \backslash\left[0, R_{1}\right] .
\end{aligned}
$$

Отсюда и из неравенств (4.5) и (4.6) следует, что

$$
h_{F}(\eta) \leqslant H_{D}(\eta)-\tilde{\varepsilon}, \quad \eta \in \mathbb{S}
$$

где $\tilde{\varepsilon}=\min \left\{\varepsilon, \varepsilon^{\prime}\right\}$. Пусть $K$ - сопряженная диаграмма функции $F$ и $L$ - замыкание множества $K+B(0, \tilde{\varepsilon} / 2)$. Тогда в силу последней оценки $L-$ компакт в области $D$. Кроме того, для некоторого $C>0$ выполнено неравенство (см. [2, с. 32])

$$
|F(\eta)| \leqslant C \exp H_{L}(\eta) \quad \forall \eta \in \mathbb{C} .
$$

Оно означает, что $F$ принадлежит пространству $P_{D}$. Следовательно, как отмечалось выше, $F$ принадлежит и множеству $I(D, \Lambda)$. По условию система (1.1) разрешима в $H(D)$ при любой допустимой правой части из $H\left(G_{1}\right) \times \cdots \times H\left(G_{n}\right)$. Тогда по лемме 1.2 найдутся функции $\varphi_{p} \in \mathbf{P}_{G_{p}}, p=1, \ldots, n$, такие, что верно тождество $F \equiv \varphi_{1} f_{1}+\cdots+\varphi_{n} f_{n}$. Согласно определению пространства $\mathbf{P}_{G_{p}}$ найдем компакт $L_{p} \subset G_{p}, p=1, \ldots, n$, и $A>0$ такие, что

$$
\left|\varphi_{p}(\eta)\right| \leqslant A \exp H_{L_{p}}(\eta), \quad \eta \in \mathbb{C}, \quad p=1, \ldots, n .
$$

Выберем $\tau>0$, удовлетворяющее неравенствам

$$
H_{L_{p}}(\eta) \leqslant H_{G_{p}}(\eta)-\tau|\eta|, \quad \eta \in \mathbb{C}, \quad p=1, \ldots, n .
$$

Тогда с учетом определений области $G_{i}$ фуункции $\psi(\eta)$ и $(4.4)$ получаем

$$
\begin{aligned}
& \left|F\left(r_{m_{j}} \eta_{0}^{*}\right)\right|=\left|\sum_{p=1}^{n} f_{p}\left(r_{m_{j}} \eta_{0}^{*}\right) \varphi_{p}\left(r_{m_{j}} \eta_{0}^{*}\right)\right| \\
& \quad \leqslant A \sum_{p=1}^{n}\left|f_{p}\left(r_{m_{j}} \eta_{0}^{*}\right)\right| \exp H_{L_{p}}\left(r_{m_{j}} \eta_{0}^{*}\right) \\
& \quad \leqslant A \sum_{p=1}^{n}\left|f_{p}\left(r_{m_{j}} \eta_{0}^{*}\right)\right| \exp \left(H_{G_{p}}\left(r_{m_{j}} \eta_{0}^{*}\right)-\tau\left|r_{m_{j}} \eta_{0}^{*}\right|\right) \\
& \quad=A \sum_{p=1}^{n}\left|f_{p}\left(r_{m_{j}} \eta_{0}^{*}\right)\right| \exp \left[\sum_{s=1, s \neq p}^{n} H_{K_{s}}\left(r_{m_{j}} \eta_{0}^{*}\right)+H_{G}\left(r_{m_{j}} \eta_{0}^{*}\right)-\tau\left|r_{m_{j}} \eta_{0}^{*}\right|\right] \\
& \leqslant A n\left|f_{i}\left(r_{m_{j}} \eta_{0}^{*}\right)\right| \exp \sum_{s=1, s \neq i}^{n}\left(H_{K_{s}}\left(r_{m_{j}} \eta_{0}^{*}\right)+H_{G}\left(r_{m_{j}} \eta_{0}^{*}\right)-\tau\left|r_{m_{j}} \eta_{0}^{*}\right|\right) .
\end{aligned}
$$


С другой стороны, в силу (4.3), определения $F(\eta)$ и выбора точек $z_{p}$ имеем

$$
\begin{aligned}
\left|F\left(r_{m_{j}} \eta_{0}^{*}\right)\right| & =\left|\varphi\left(r_{m_{j}} \eta_{0}^{*}\right)\right|\left|f_{i}\left(r_{m_{j}} \eta_{0}^{*}\right)\right|\left|\prod_{p=1, p \neq i}^{n} \exp \left(z_{p} r_{m_{j}} \eta_{0}^{*}\right)\right| \\
& =\left|f_{i}\left(r_{m_{j}} \eta_{0}^{*}\right)\right|\left|\varphi\left(r_{m_{j}} \eta_{0}^{*}\right)\right| \exp \sum_{p=1, s \neq i}^{n} H_{K_{p}}\left(r_{m_{j}} \eta_{0}^{*}\right) \\
& =\left|f_{i}\left(r_{m_{j}} \eta_{0}^{*}\right)\right| \exp \sum_{s=1, s \neq i}^{n}\left(H_{K_{s}}\left(r_{m_{j}} \eta_{0}^{*}\right)+H_{G}\left(r_{m_{j}} \eta_{0}^{*}\right)-\tau_{j}\left|r_{m_{j}} \eta_{0}^{*}\right|\right),
\end{aligned}
$$

где $\tau_{j} \rightarrow 0$, когда $j \rightarrow \infty$. Это противоречит последнему неравенству. Следовательно, наше предположение о том, что $\psi(\eta)$ не имеет регулярного роста на луче $t \eta^{\prime}$, неверно. Предложение доказано.

ЗАмечАниЕ 4.1. Можно показать, что при условии разрешимости системы (1.1) функция $\psi(\eta)$ будет иметь регулярный рост не только в направлениях гладкости области $G$. Регулярный рост будет иметь место, например, в направлениях $\eta$, удовлетворяюших следующему условию: по крайней мере два компакта из совокупности $K_{i}, i=1, \ldots, n$, имеют дважды гладкую границу в окрестности точек $z \in \partial K_{i}$ таких, что $\eta \in \Gamma(z)$.

Теорема 4.4. Пусть система (1.1) разрешима в $H(D)$ для любой допустимой правой части из $H\left(G_{1}\right) \times \cdots \times H\left(G_{n}\right)$. Если для некоторого $\eta_{0} \in \mathbb{S} \backslash I_{G}$ функиия $\psi(\xi)$ имеет регулярный рост на луче $t \eta_{0}$, то система функций $\left(f_{1}, \ldots, f_{n}\right)$ также имеет регулярный рост на луче $t \eta_{0}$.

ДоКАЗАТЕЛЬСТво. Пусть $\eta_{0} \in \mathbb{S} \backslash I_{G}$ и функция $\psi(\xi)$ имеет регулярный рост на луче $t \eta_{0}$. Тогда согласно определению 2.1 найдется последовательность $\left\{z_{k}\right\}_{k=1}^{\infty} \in$ $\mathbf{E}\left(\eta_{0}\right)$ такая, что

$$
\lim _{k \rightarrow \infty} \frac{\psi\left(z_{k}\right)}{\left|z_{k}\right|}=h_{\psi}\left(\eta_{0}\right)
$$

Как и в $(2.4)$, для каждого $m=1,2, \ldots$ найдем $R_{m}, \delta_{m}>0$ такие, что

$$
\frac{\ln \left|f_{i}(t z)\right|}{t} \leqslant h_{f_{i}}\left(\eta_{0}\right)+2^{-m}, \quad z \in B\left(\eta_{0}, 6 \delta_{m}\right), \quad t \geqslant R_{m}, \quad i=1, \ldots, n
$$

Можно считать, что $\delta_{m} \rightarrow 0$, когда $m \rightarrow \infty$. Пусть $\varepsilon>0$ и $2^{-m} \leqslant \varepsilon$ при $m \geqslant m_{0}$. Покажем, что для некоторого $m \geqslant m_{0}$ существует номер $k_{0}$ такой, что выполнены неравенства

$$
\max _{1 \leqslant i \leqslant n}\left[\ln \left|\frac{f_{i}(z)}{q_{\delta_{m}}\left(z, z_{k}, \Lambda\right)}\right|-h_{f_{i}}(z)\right] \geqslant-\varepsilon|z|, \quad z \in B\left(z_{k}, 4^{-1} \delta_{m}\left|z_{k}\right|\right), \quad k \geqslant k_{0} .
$$

Предположим, что нам удалось это сделать. Тогда согласно предложению 2.2 система $\left(f_{1}, \ldots, f_{n}\right)$ имеет регулярньй рост на луче $t \eta_{0}$. Действительно, $(4.9)$ обеспечивает выполнение (2.7), а (4.8) - выполнение (2.8). Наконец, (2.9) легко следует из (4.7) и определения функции $\psi(\eta)$. 
Приведем доказательство неравенства (4.9) от противного. Предположим, что оно неверно. Тогда с учетом (4.7) для каждого $m \geqslant m_{0}$ найдем номер $k_{m}>m$ и точку $z_{m} \in B\left(z_{k_{m}}, 4^{-1} \delta_{m}\left|z_{k_{m}}\right|\right)$ такие, что $\delta_{m}\left|z_{k_{m}}\right| \geqslant 1$,

$$
\begin{gathered}
\frac{\psi\left(z_{k_{m}}\right)}{\left|z_{k_{m}}\right|} \geqslant h_{\psi}\left(\eta_{0}\right)-2^{-m} \\
\max _{1 \leqslant i \leqslant n}\left[\ln \left|\frac{f_{i}\left(z_{m}\right)}{q_{\delta_{m}}\left(z_{m}, z_{k_{m}}, \Lambda\right)}\right|-h_{f_{i}}\left(z_{m}\right)\right]<-\varepsilon\left|z_{m}\right| .
\end{gathered}
$$

Из неравенства (4.10) и определения функции $\psi(\eta)$ получаем

$$
\max _{1 \leqslant p \leqslant n}\left[\frac{\ln \left|f_{p}\left(z_{k_{m}}\right)\right|}{\left|z_{k_{m}}\right|}-h_{f_{p}}\left(\eta_{0}\right)\right] \geqslant-2^{-m}
$$

Переходя к подпоследовательности, можно считать, что для некоторого $i=1, \ldots, n$ и всех $m \geqslant m_{0}$ максимум здесь достигается при $p=i$. Тогда

$$
\frac{\ln \left|f_{i}\left(z_{k_{m}}\right)\right|}{\left|z_{k_{m}}\right|} \geqslant h_{f_{i}}\left(\eta_{0}\right)-2^{-m}, \quad m \geqslant m_{0}
$$

Отсюда с учетом (4.8) имеем

$$
\ln \left[\frac{\left|f_{i}(z)\right|}{\left|f_{i}\left(z_{k_{m}}\right)\right|}\right] \leqslant 2^{-m+1}\left|z_{k_{m}}\right|, \quad z \in B\left(z_{k_{m}}, 6 \delta_{m}\left|z_{k_{m}}\right|\right), \quad m \geqslant m_{1},
$$

где номер $m_{1} \geqslant m_{0}$ выбран так, что $\left|z_{k_{m}}\right| \geqslant R_{m}$ для всех $m \geqslant m_{1}$. Следовательно, по теореме об оценке снизу модуля аналитической функции (см. [15, с. 39]) для любого $m \geqslant m_{1}$ верно неравенство

$$
\ln \left[\frac{\left|f_{i}(z)\right|}{\left|f_{i}\left(z_{k_{m}}\right)\right|}\right] \geqslant-\tau 2^{-m+1}\left|z_{k_{m}}\right|, \quad \tau=3+\ln 48,
$$

которое выполнено в круге $B\left(z_{k_{m}}, \delta_{m}\left|z_{k_{m}}\right|\right)$, но вне исключительных кружков с обшей суммой радиусов, равной $8^{-1} \delta_{m}\left|z_{k_{m}}\right|$. Поскольку сумма диаметров этих кружков равна $4^{-1} \delta_{m}\left|z_{k_{m}}\right|$, то для каждого $m \geqslant m_{1}$ найдется $r_{m} \in[1 / 2,3 / 4]$ такое, что

$$
\ln \left[\frac{\left|f_{i}(z)\right|}{\left|f_{i}\left(z_{k_{m}}\right)\right|}\right] \geqslant-\tau 2^{-m+1}\left|z_{k_{m}}\right|, \quad z \in \partial B\left(z_{k_{m}}, r_{m} \delta_{m}\left|z_{k_{m}}\right|\right) .
$$

Отсюда с учетом (4.12) получаем

$\ln \left|f_{i}(z)\right| \geqslant h_{f_{i}}\left(z_{k_{m}}\right)-2^{-m}(1+2 \tau)\left|z_{k_{m}}\right|, \quad z \in \partial B\left(z_{k_{m}}, r_{m} \delta_{m}\left|z_{k_{m}}\right|\right), \quad m \geqslant m_{1}$. 
Так как $\delta_{m}\left|z_{k_{m}}\right| \geqslant 1$, то, представляя производную $f_{i}^{\prime}$ интегралом Коши по единичной окружности с центром в точке $z \in B\left(z_{k_{m}}, 5 \delta_{m}\left|z_{k_{m}}\right|\right)$ и учитывая (4.8), имеем оценку

$$
\left|f_{i}^{\prime}(z)\right| \leqslant \exp \left[h_{f_{i}}\left(z_{k_{m}}\right)+2^{-m}\left|z_{k_{m}}\right|\right], \quad z \in B\left(z_{k_{m}}, 5 \delta_{m}\left|z_{k_{m}}\right|\right), \quad m \geqslant m_{1}
$$

Отсюда следует, что для любых $y, z \in B\left(z_{k_{m}}, 2 \delta_{m}\left|z_{k_{m}}\right|\right)$ верно неравенство

$$
\left|f_{i}(z)-f_{i}(y)\right| \leqslant \int_{[z, y]}\left|f_{i}^{\prime}(\xi)\right||d \xi| \leqslant \exp \left[h_{f_{i}}\left(z_{k_{m}}\right)+2^{-m}\left|z_{k_{m}}\right|\right]|z-y| .
$$

Пусть точка $z \in \partial B\left(z_{k_{m}}, r_{m} \delta_{m}\left|z_{k_{m}}\right|\right)$ и точка $y \in B\left(z, \alpha_{m}\right)$, где $\alpha_{m}=4^{-1} \times$ $\exp \left[-(3+2 \tau) 2^{-m}\left|z_{k_{m}}\right|\right]$. Тогда в силу (4.13) имеем

$$
\begin{aligned}
\left|f_{i}(y)\right| & \geqslant\left|f_{i}(z)\right|-\exp \left[h_{f_{i}}\left(z_{k_{m}}\right)+2^{-m}\left|z_{k_{m}}\right|\right]|z-y| \\
& \geqslant \exp \left[h_{f_{i}}\left(z_{k_{m}}\right)-(1+2 \tau) 2^{-m}\left|z_{k_{m}}\right|\right]\left(1-4^{-1} \exp \left[-2^{-m}\left|z_{k_{m}}\right|\right]\right) .
\end{aligned}
$$

Согласно определению опорной функции найдем точку $y_{0} \in D$ такую, что

$$
\operatorname{Re}\left[y_{0} \eta_{0}\right] \geqslant H_{D}\left(\eta_{0}\right)-\frac{\varepsilon}{2}
$$

Так как $D=G_{i}+K_{i}$, то в области $G_{i}$ найдется компакт $L_{i}$ такой, что $L_{i}+K_{i}$ содержит $y_{0}$. Тогда

$$
\operatorname{Re}\left[y_{0} \eta\right] \leqslant H_{L_{i}}(\eta)+H_{K_{i}}(\eta) \quad \forall \eta
$$

Пусть $\varepsilon^{\prime}>0$ удовлетворяет условию

$$
H_{L_{i}}(\eta)+10 \varepsilon^{\prime}|\eta| \leqslant H_{G_{i}}(\eta) \quad \forall \eta
$$

Выберем номер $m_{2} \geqslant m_{1}$ такой, что для всех $m \geqslant m_{2}$ имеет место оценка

$$
h_{f_{i}}\left(z_{k_{m}}\right)-(1+2 \tau) 2^{-m}\left|z_{k_{m}}\right| \geqslant h_{f_{i}}(z)-\varepsilon^{\prime}|z|, \quad z \in B\left(z_{k_{m}}, 2 \delta_{m}\left|z_{k_{m}}\right|\right) .
$$

Это можно сделать в силу непрерывности и однородности опорной функции, а также того, что $\delta_{m} \rightarrow 0$. Тогда из (4.14) получаем

$$
\begin{gathered}
\left|f_{i}(z)\right| \geqslant 2^{-1} \exp \left[h_{f_{i}}(z)-\varepsilon^{\prime}|z|\right] \\
\forall z \in B\left(z_{k_{m}}, r_{m} \delta_{m}\left|z_{k_{m}}\right|+\alpha_{m}\right) \backslash B\left(z_{k_{m}}, r_{m} \delta_{m}\left|z_{k_{m}}\right|-\alpha_{m}\right), \quad m \geqslant m_{2} .
\end{gathered}
$$

Пусть $k(r)$ - число точек $z_{m}$, попавших в круг $B(0, r)$. Переходя к подпоследовательности, можно считать, что круги $B\left(z_{k_{m}}, 2 \delta_{m}\left|z_{k_{m}}\right|\right), m \geqslant m_{2}$, попарно не пересекаются и выполнено следующее:

$$
\lim _{r \rightarrow \infty} \frac{k(r)}{r}=0, \quad \sum_{m=1}^{\infty} \frac{1}{\left|z_{m}\right|}<\infty
$$


Тогда функция $\theta(z)=\prod_{m=m_{2}}^{\infty}\left(1-z / z_{m}\right)$ является целой и имеет первый порядок и минимальньй тип (см. [1]). Нули этой функции совпадают с последовательностью $\left\{z_{m}\right\}$. Кроме того, она имеет регулярный рост и регулярное нулевое множество (поскольку $\delta_{m}\left|z_{k_{m}}\right| \geqslant 1$, то ||$z_{m}|-| z_{j}|| \geqslant 2^{-1}$ для любых $j, m \geqslant m_{1}$ ). Следовательно, для каждого $\tilde{\varepsilon}>0$ найдется $R>0$ такое, что верна оценка (см. [1])

$$
|\theta(z)| \geqslant \exp [-\tilde{\varepsilon}|z|] \quad \forall z:|z| \geqslant R, \quad z \notin \bigcup_{m=m_{2}}^{\infty} B\left(z_{m}, 4^{-1}\right)
$$

В частности, поскольку $B\left(z_{m}, 4^{-1}\right) \subset B\left(z_{m}, 4^{-1} \delta_{m}\left|z_{k_{m}}\right|\right) \subset B\left(z_{k_{m}}, 2^{-1} \delta_{m}\left|z_{k_{m}}\right|\right)$, найдется номер $m_{3} \geqslant m_{2}$ такой, что

$$
\begin{gathered}
|\theta(z)| \geqslant \exp \left[-\varepsilon^{\prime}|z|\right], \\
z \in B\left(z_{k_{m}}, r_{m} \delta_{m}\left|z_{k_{m}}\right|+\alpha_{m}\right) \backslash B\left(z_{k_{m}}, r_{m} \delta_{m}\left|z_{k_{m}}\right|\right), \quad m \geqslant m_{3} .
\end{gathered}
$$

Для каждого $m \geqslant m_{3}$ рассмотрим функцию $q_{\delta_{m}}\left(z, z_{k_{m}}, \Lambda\right)$. Имеем

$$
\left|q_{\delta_{m}}\left(z, z_{k_{m}}, \Lambda\right)\right| \leqslant \prod \frac{\left(3 \delta_{m}\left|z_{k_{m}}\right|\right)^{l_{j}}}{\left|z_{k_{m}}-\lambda_{j}\right|^{l_{j}}}, \quad z \in B\left(z_{k_{m}}, 2 \delta_{m}\left|z_{k_{m}}\right|\right)
$$

где $\Lambda=\left\{\lambda_{j}, l_{j}\right\}$ - последовательность общих нулей и их кратностей функций $f_{1}, \ldots, f_{n}$, а произведение берется по всем $j$ таким, что $\lambda_{j} \in B\left(z_{k_{m}}, 2 \delta_{m}\left|z_{k_{m}}\right|\right)$. Функция $f_{i}$ обрашается в нуль во всех точках $\lambda_{j}$ с кратностью, не меньшей чем $l_{j}$. Поэтому из $(4.8),(4.12)$ и теоремы о нулях аналитической функции в круге (см. [15, с. 16]) следует, что

$$
\prod \frac{\left(3 \delta_{m}\left|z_{k_{m}}\right|\right)^{l_{j}}}{\left|z_{k_{m}}-\lambda_{j}\right|^{l_{j}}} \leqslant \frac{\exp \left[h_{f_{i}}\left(z_{k_{m}}\right)+2^{-m}\left|z_{k_{m}}\right|\right]}{\exp \left[h_{f_{i}}\left(z_{k_{m}}\right)-2^{-m}\left|z_{k_{m}}\right|\right]}=\exp \left[2^{-m+1}\left|z_{k_{m}}\right|\right] .
$$

Выберем номер $m_{4} \geqslant m_{3}$ такой, что при $m \geqslant m_{4}$

$$
\exp \left[2^{-m+1}\left|z_{k_{m}}\right|\right] \leqslant \exp \left[\varepsilon^{\prime}|z|\right], \quad z \in B\left(z_{k_{m}}, 2 \delta_{m}\left|z_{k_{m}}\right|\right) .
$$

Тогда из предыдушего получаем

$$
\left|q_{\delta_{m}}\left(z, z_{k_{m}}, \Lambda\right)\right| \leqslant \exp \left[\varepsilon^{\prime}|z|\right], \quad z \in B\left(z_{k_{m}}, 2 \delta_{m}\left|z_{k_{m}}\right|\right) .
$$

Выберем $m_{5} \geqslant m_{4}$ такое, что при $m \geqslant m_{5}$

$$
\alpha_{m} \geqslant 4^{-1} \exp \left[-\varepsilon^{\prime}|z|\right], \quad z \in B\left(z_{k_{m}}, 2 \delta_{m}\left|z_{k_{m}}\right|\right) .
$$

Пусть $e(z)$ - функция, построенная в лемме 3.1 , где полагаем $A=\varepsilon^{\prime}, a=8^{-1}$,

$$
\Omega=\bigcup_{m=m_{5}}^{\infty} B\left(z_{k_{m}}, r_{m} \delta_{m}\left|z_{k_{m}}\right|+\alpha_{m}\right), \quad K=\bigcup_{m=m_{5}}^{\infty} \overline{B\left(z_{k_{m}}, r_{m} \delta_{m}\left|z_{k_{m}}\right|\right)} .
$$

Через $q(z)$ обозначим функцию, которая всюду в $\mathbb{C}$ вне объединения $\bigcup_{m=m_{5}}^{\infty} B\left(z_{k_{m}}, 2 \delta_{m}\left|z_{k_{m}}\right|\right)$ равна нулю, а в каждом круге $B\left(z_{k_{m}}, 2 \delta_{m}\left|z_{k_{m}}\right|\right)$, где 
$m \geqslant m_{5}$, совпадает с функцией $q_{\delta_{m}}\left(z, z_{k_{m}}, \Lambda\right)$. Это определение корректно, так как круги $B\left(z_{k_{m}}, 2 \delta_{m}\left|z_{k_{m}}\right|\right), m \geqslant m_{5}$, попарно не пересекаются. По лемме 3.1 функция $e(z) q(z)$ бесконечно дифференцируема во всей плоскости, равна нулю вне $\Omega$ и совпадает с $q_{\delta_{m}}\left(z, z_{k_{m}}, \Lambda\right)$ на множестве $K$. Из оценок $(4.16),(4.20)$ и неравенства на производную функцию $e(z)$ в лемме 3.1 получаем

$$
\left|\frac{d e}{d \bar{z}}(z) q(z) \exp \left(y_{0} z\right)\right| \leqslant \exp \left[H_{L_{i}}(z)+H_{K_{i}}(z)+2 \varepsilon^{\prime}|z|\right] \quad \forall z .
$$

Согласно лемме 3.1 функция $e(z)$ постоянна всюду в плоскости, за исключением колец $B\left(z_{k_{m}}, r_{m} \delta_{m}\left|z_{k_{m}}\right|+\alpha_{m}\right) \backslash B\left(z_{k_{m}}, r_{m} \delta_{m}\left|z_{k_{m}}\right|\right)$, поэтому ее производная равна нулю вне этих колец. Следовательно, с учетом (4.18) и (4.19) верна оценка

$$
\left|\frac{d e}{d \bar{z}}(z) q(z) \exp \left(y_{0} z\right) f_{i}^{-1}(z) \theta^{-1}(z)\right| \leqslant \exp \left[H_{L_{i}}(z)+4 \varepsilon^{\prime}|z|\right] \quad \forall z,
$$

которая влечет за собой сходимость интеграла

$$
\int_{\mathbb{C}}\left|q(z) \exp \left(y_{0} z\right) f_{i}^{-1}(z) \theta^{-1}(z) \frac{d e}{d \bar{z}}(z)\right|^{2} \exp \left(-2 H_{L_{i}}(z)-10 \varepsilon^{\prime}|z|\right) d \sigma
$$

Тогда из $\left[17\right.$, теорема 4.4.2] следует, что существует функция $\nu \in C^{\infty}$, являюшаяся решением $\bar{\partial}$-уравнения

$$
\frac{d \nu}{d \bar{z}}(z)=\frac{d e}{d \bar{z}}(z) q(z) \exp \left(y_{0} z\right) f_{i}^{-1}(z) \theta^{-1}(z)
$$

и удовлетворяющая интегральной оценке

$$
\int_{\mathbb{C}}|\nu(z)|^{2} \exp \left(-2 H_{L_{i}}(z)-12 \varepsilon^{\prime}|z|\right) d \sigma<\infty .
$$

Рассмотрим функцию $F(z)=e(z) q(z) \exp \left(y_{0} z\right)-\nu(z) f_{i}(z) \theta(z)$. В силу (4.21) эта функция является целой. Покажем, что $F \in \mathbf{P}_{D}$. Поскольку $0 \leqslant e(z) \leqslant 1$ и $e(z)$ равна нулю вне $\Omega$, то из (4.16) и (4.20) следует, что

$$
\left|e(z) q(z) \exp \left(y_{0} z\right)\right| \leqslant \exp \left[H_{L_{i}}(z)+H_{K_{i}}(z)+\varepsilon^{\prime}|z|\right] \quad \forall z .
$$

Это неравенство влечет за собой интегральное неравенство

$$
\int_{\mathbb{C}}\left|e(z) q(z) \exp \left(y_{0} z\right)\right|^{2} \exp \left[-2 H_{L_{i}}(z)-H_{K_{i}}(z)-4 \varepsilon^{\prime}|z|\right] d \sigma<\infty .
$$

Поскольку $K_{i}$ - сопряженная диаграмма функции $f_{i}$, то найдется $B>0$ такое, что верно неравенство (см. $[2$, с. 32$])$

$$
\left|f_{i}(z)\right| \leqslant B \exp \left(H_{K_{i}}(z)+\varepsilon^{\prime}|z|\right) \quad \forall z
$$

Функция $\theta(z)$ имеет минимальный тип. Поэтому для некоторого $B_{1}>0$ имеем

$$
|\theta(z)| \leqslant B_{1} \exp \left(\varepsilon^{\prime}|z|\right) \quad \forall z .
$$


Из двух последних неравенств и (4.22) следует, что

$$
\int_{\mathbb{C}}\left|\nu(z) f_{i}(z) \theta(z)\right|^{2} \exp \left(-2 H_{L_{i}}(z)-2 H_{K_{i}}(z)-16 \varepsilon^{\prime}|z|\right) d \sigma<\infty .
$$

Отсюда с учетом (4.23) и неравенства Коши-Буняковского легко получаем

$$
\int_{\mathbb{C}}|F(z)|^{2} \exp \left(-2 H_{L_{i}}(z)-2 H_{K_{i}}(z)-16 \varepsilon^{\prime}|z|\right) d \sigma<\infty .
$$

Точно так же, как и при оценке функции $\varphi_{i}(y)$ в теореме 3.7 , сходимость этого интеграла влечет за собой неравенство

$$
|F(z)| \leqslant B_{2} \exp \left(H_{L_{i}}(z)+H_{K_{i}}(z)+9 \varepsilon^{\prime}|z|\right) \quad \forall z
$$

где $B_{2}>0$ не зависит от $z$. Пусть $L$ - замыкание множества $L_{i}+K_{i}+B\left(0,9 \varepsilon^{\prime}\right)$. Из (4.17) следует, что компакт $L$ лежит в области $D$. Но тогда последняя оценка означает, что $F \in \mathbf{P}_{D}$.

Докажем теперь, что $F \in I(D, \Lambda)$. Для этого нужно показать, что $F$ обращается в нуль во всех точках $\lambda_{j}$ с кратностью, не меньшей чем $l_{j}$. Согласно определению последовательности $\Lambda=\left\{\lambda_{j}, l_{j}\right\}$ этим свойством обладает функция $f_{i}$. Поэтому достаточно показать, что то же самое верно и для функции $e(z) q(z)$. Если $\lambda_{j} \notin \Omega$, то требуемое следует из того, что $e(z)$ и все ее производные равны нулю вне $\Omega$. Если же $\lambda_{j} \in \Omega$, то по определению функции $q(z)$ она обращается в нуль в $\lambda_{j}$ с кратностью $l_{j}$. Таким образом, $F \in I(\Delta, \Lambda)$.

По условию система (1.1) разрешима в $H(D)$ при любой допустимой правой части из $H\left(G_{1}\right) \times \cdots \times H\left(G_{n}\right)$. Следовательно, по лемме 1.2 сушествуют функции $\varphi_{p} \in \mathbf{P}_{G_{p}}, p=1, \ldots, n$, такие, что $F \equiv \varphi_{1} f_{1}+\cdots+\varphi_{n} f_{n}$. Согласно определению пространства $\mathbf{P}_{G_{p}}$ для каждого $p=1, \ldots, n$ найдем компакт $T_{p} \subset G_{p}$, которьй для некоторого $C_{p}>0$ удовлетворяет неравенству

$$
\left|\varphi_{p}(z)\right| \leqslant C_{p} \exp H_{T_{p}}(z) \quad \forall z .
$$

Как и ранее, найдем номер $m_{6} \geqslant m_{5}$ такой, что при $m \geqslant m_{6}$

$$
\begin{gathered}
H_{K_{p}}\left(z_{m}\right)+H_{T_{p}}\left(z_{m}\right)<\left(H_{K_{p}}\left(\eta_{0}\right)+H_{T_{p}}\left(\eta_{0}\right)+\frac{\varepsilon}{5}\right)\left|z_{m}\right|, \quad p=1, \ldots, n \\
\operatorname{Re}\left(y_{0} z_{m}\right)>\left(\operatorname{Re}\left(y_{0} \eta_{0}\right)-\frac{\varepsilon}{4}\right)\left|z_{m}\right| .
\end{gathered}
$$

Согласно (4.11), (4.24) и (4.25) для каждого $m \geqslant m_{6}$ получаем

$$
\begin{aligned}
\left|\frac{F\left(z_{m}\right)}{q_{\delta_{m}}\left(z_{m}, z_{k_{m}}, \Lambda\right)}\right| & \leqslant \sum_{p=1}^{n}\left|\frac{\varphi_{p}\left(z_{m}\right) f_{p}\left(z_{m}\right)}{q_{\delta_{m}}\left(z_{m}, z_{k_{m}}, \Lambda\right)}\right| \\
& \leqslant \sum_{p=1}^{n} C_{p} \exp \left(H_{T_{p}}\left(z_{m}\right)+H_{K_{p}}\left(z_{m}\right)-\varepsilon\left|z_{m}\right|\right) \\
& <\sum_{p=1}^{n} C_{p} \exp \left(\left(H_{T_{p}}\left(\eta_{0}\right)+H_{K_{p}}\left(\eta_{0}\right)-\frac{4 \varepsilon}{5}\right)\left|z_{m}\right|\right) \\
& \leqslant \sum_{p=1}^{n} C_{p} \exp \left(\left(H_{G_{p}}\left(\eta_{0}\right)+H_{K_{p}}\left(\eta_{0}\right)-\frac{4 \varepsilon}{5}\right)\left|z_{m}\right|\right) \\
& =C \exp \left(\left(H_{D}\left(\eta_{0}\right)-\frac{4 \varepsilon}{5}\right)\left|z_{m}\right|\right)
\end{aligned}
$$


где $C=C_{1}+\cdots+C_{n}$. С другой стороны, в точках $z_{m}, m \geqslant m_{6}$, функция $F(z)$ совпадает с функцией $q_{\delta_{m}}\left(z, z_{k_{m}}, \Lambda\right) \exp \left(y_{0} z\right)$, поскольку $\theta\left(z_{m}\right)=0$ и $e\left(z_{m}\right)=1$. Поэтому из (4.15) и (4.26) получаем

$$
\begin{aligned}
\left|\frac{F\left(z_{m}\right)}{q_{\delta_{m}}\left(z_{m}, z_{k_{m}}, \Lambda\right)}\right| & =\left|\exp \left(y_{0} z_{m}\right)\right|=\exp \operatorname{Re}\left(y_{0} z_{m}\right) \\
& >\exp \left(\operatorname{Re}\left(y_{0} \eta_{0}\right)-\frac{3 \varepsilon}{4}\right)\left|z_{m}\right| \geqslant \exp \left(H_{D}\left(\eta_{0}\right)-\frac{3 \varepsilon}{4}\right)\left|z_{m}\right|
\end{aligned}
$$

Это неравенство противоречит предыдушей оценке. Таким образом, (4.9) верно и теорема доказана.

TеОРема 4.5. Пусть $D$ - неограниченная область и система (1.1) разрешима в $H(D)$ при любой допустимой правой части из $H\left(G_{1}\right) \times \cdots \times H\left(G_{n}\right)$. Тогда система $\left(f_{1}, \ldots, f_{n}\right)$ имеет слабый регулярный рост на каждом луие uз int $I_{G}$.

ДокАЗАТЕЛЬСТво. Пусть $\eta_{0} \in \mathbb{S} \cap \operatorname{int} I_{G}$ и $r>\tau>1$ фиксированы. Выберем $\delta_{0}>0$ такое, что круг $B\left(\eta_{0}, 3 r \delta_{0}\right)$ компактно принадлежит $I_{G}$, и пусть $0<\delta \leqslant \delta_{0}$. По теореме об оценке снизу на окружностях целой функции конечного порядка и типа (см. $[15$, с. 40$])$ найдем последовательность $\left\{z_{k}\right\} \in \mathbf{E}\left(\eta_{0}, \delta\right)$ и число $A>0$ такие, что

$$
\ln \left|f_{1}\left(z_{k}\right)\right| \geqslant A\left|z_{k}\right|, \quad k=1,2, \ldots
$$

Согласно предложению 2.3 , чтобы доказать слабую регулярность роста системы $\left(f_{1}, \ldots, f_{n}\right)$ на луче $t \eta_{0}$, достаточно показать, что для некоторых $k_{0}$ и $B>0$ выполнены неравенства

$$
\max _{1 \leqslant i \leqslant n} \ln \left|\frac{f_{i}(z)}{q_{3 r \delta}\left(z, z_{k}, \Lambda\right)}\right| \geqslant-B|z|, \quad z \in B\left(z_{k}, \tau \delta\left|z_{k}\right|\right), \quad k \geqslant k_{0} .
$$

Предположим, что это неверно. Тогда найдутся последовательности $\left\{B_{m}\right\}$, $B_{m} \rightarrow+\infty,\left\{z_{m}\right\}$ и подпоследовательность $\left\{k_{m}\right\}$ такие, что $z_{m} \in B\left(z_{k_{m}}, \tau \delta\left|z_{k_{m}}\right|\right)$ И

$$
\max _{1 \leqslant i \leqslant n} \ln \left|\frac{f_{i}\left(z_{m}\right)}{q_{3 r \delta}\left(z_{m}, z_{k_{m}}, \Lambda\right)}\right|<-B_{m}\left|z_{m}\right|, \quad m=1,2, \ldots
$$

При этом можно считать, что круги $B\left(z_{k_{m}}, 3 r \delta\left|z_{k_{m}}\right|\right), m \geqslant 1$, попарно не пересекаются, и $\delta\left|z_{k_{m}}\right| \geqslant 1, m \geqslant 1$. Поскольку $f_{1}$ является функцией экспоненциального типа, то для некоторого $A_{1}>0$ выполнены неравенства

$$
\ln \left|f_{1}(z)\right| \leqslant A_{1}\left|z_{k_{m}}\right|, \quad z \in B\left(z_{k_{m}}, 18 r \delta\left|z_{k_{m}}\right|\right), \quad m \geqslant m_{1} .
$$

Пользуясь неравенствами $(4.27),(4.30)$ и проводя такие же рассуждения, как и в теореме 4.4, получим

$$
\begin{gathered}
\left|f_{1}(z)\right| \geqslant 2^{-1} \exp \left[-A_{2}|z|\right] \\
\forall z \in B\left(z_{k_{m}}, r_{m} r \delta\left|z_{k_{m}}\right|+\alpha_{m}\right) \backslash B\left(z_{k_{m}}, r_{m} r \delta\left|z_{k_{m}}\right|-\alpha_{m}\right), \quad m \geqslant m_{2},
\end{gathered}
$$


где $r_{m} \in[1,2]$ и $\alpha_{m}=2^{-1} \exp \left[-A_{3}\left|z_{k_{m}}\right|\right], A_{3}>0$. Далее по лемме 3.1 строим функцию $e(z) \in C^{\infty}$, которая на множестве $\bigcup_{m=m_{2}}^{\infty} B\left(z_{k_{m}}, r_{m} r \delta\left|z_{k_{m}}\right|\right)$ равна единище, вне объединения кругов $B\left(z_{k_{m}}, r r_{m} \delta\left|z_{k_{m}}\right|+\alpha_{m}\right), m \geqslant m_{2}$, равна нулю и для некоторого $A_{4}>0$ удовлетворяет неравенству

$$
\left|\frac{d e(z)}{d \bar{z}}\right| \leqslant A_{4}|z| \quad \forall z
$$

Как и при получении оценки (4.20), из теоремы о нулях аналитической функции в круге следует, что для некоторых $A_{5}>0$ и $m_{3} \geqslant m_{2}$ верно неравенство

$$
\left|q_{3 r \delta}\left(z, z_{k_{m}}, \Lambda\right)\right| \leqslant \exp \left[A_{5}|z|\right], \quad z \in B\left(z_{k_{m}}, 3 r \delta\left|z_{k_{m}}\right|\right), \quad m \geqslant m_{3} .
$$

Пусть функция $\theta(z)$ построена в теореме 4.4 по точкам $z_{m}$. Так же, как и в $(4.19)$, получаем

$$
\begin{gathered}
|\theta(z)| \geqslant \exp \left[-A_{6}|z|\right], \\
z \in B\left(z_{k_{m}}, r r_{m} \delta\left|z_{k_{m}}\right|+\alpha_{m}\right) \backslash B\left(z_{k_{m}}, r r_{m} \delta\left|z_{k_{m}}\right|\right), \quad m \geqslant m_{4},
\end{gathered}
$$

где $A_{6}>0$. Пусть $q(z)$ равна нулю вне кругов $B\left(z_{k_{m}}, 3 r \delta\left|z_{k_{m}}\right|\right), m \geqslant m_{4}$, а в каждом из $B\left(z_{k_{m}}, 3 r \delta\left|z_{k_{m}}\right|\right), m \geqslant m_{4}$, совпадает с функцией $q_{3 r \delta}\left(z, z_{k_{m}}, \Lambda\right)$. Выберем произвольную точку $y_{0} \in D$ такую, что $\operatorname{Re}\left(y_{0} \eta_{0}\right)>0$ (это можно сделать, так как $\left.H_{G}\left(\eta_{0}\right)=H_{D}\left(\eta_{0}\right)=+\infty\right)$. Уменьшая при необходимости $\delta_{0}>0$, можно считать, что $\operatorname{Re}\left(y_{0} \eta\right)>0 \forall \eta \in B\left(\eta_{0}, 3 r \delta_{0}\right)$. Тогда

$$
\operatorname{Re}\left(y_{0} z_{m}\right)>0, \quad m=1,2, \ldots
$$

Из (4.31)-(4.34) следует, что для некоторого $A_{7}>0$ выполнено неравенство

$$
\begin{gathered}
\left|\frac{d e}{d \bar{z}}(z) q(z) \exp \left(y_{0} z\right) f_{1}^{-1}(z) \theta^{-1}(z)\right| \leqslant \exp \left[A_{7}|z|\right], \\
z \in B\left(z_{k_{m}}, r r_{m} \delta\left|z_{k_{m}}\right|+\alpha_{m}\right) \backslash B\left(z_{k_{m}}, r r_{m} \delta\left|z_{k_{m}}\right|\right), \quad m \geqslant m_{4} .
\end{gathered}
$$

В силу выбора $\delta_{0}$ круг $B\left(\eta_{0}, 3 r \delta\right)$ компактно принадлежит множеству $I_{G}=I_{G_{1}}$. Тогда согласно определению опорной функции для каждой точки $z$ из $\overline{B\left(\eta_{0}, 3 r \delta\right)}$ найдется $y(z) \in G_{1}$ такое, что $\operatorname{Re}(y(z), z)>A_{7}|z|$. По непрерывности это неравенство будет выполнено в некоторой окрестности $U(z)$ точки $z$. Выделим из покрытия $\overline{B\left(\eta_{0}, 3 r \delta\right)}$ множествами $U(z), z \in \overline{B\left(\eta_{0}, 3 r \delta\right)}$, конечное подпокрытие $U\left(z_{s}\right)$, $s=1, \ldots, k$. Пусть $L_{1}-$ выпуклая оболочка объединения $\bigcup_{s=1}^{k}\left\{y\left(z_{s}\right)\right\}$. Тогда компакт $L_{1}$ лежит в области $G_{1}$, и в силу (4.36) и вложения $B\left(z_{k_{m}}, r r_{m} \delta\left|z_{k_{m}}\right|+\right.$ $\left.\alpha_{m}\right) \subset B\left(z_{k_{m}}, 3 r \delta\left|z_{k_{m}}\right|\right)$ выполнено неравенство

$$
\begin{gathered}
\left|\frac{d e}{d \bar{z}}(z) q(z) \exp \left(y_{0} z\right) f_{1}^{-1}(z) \theta^{-1}(z)\right| \leqslant \exp \left[H_{L_{1}}|z|\right], \\
z \in B\left(z_{k_{m}}, r r_{m} \delta\left|z_{k_{m}}\right|+\alpha_{m}\right) \backslash B\left(z_{k_{m}}, r r_{m} \delta\left|z_{k_{m}}\right|\right), \quad m \geqslant m_{4} .
\end{gathered}
$$


Так как функция $q(z) \frac{d e}{d \bar{z}}(z)$ не равна нулю лишь в кольцах

$$
B\left(z_{k_{m}}, r r_{m} \delta\left|z_{k_{m}}\right|+\alpha_{m}\right) \backslash B\left(z_{k_{m}}, r r_{m} \delta\left|z_{k_{m}}\right|\right), \quad m \geqslant m_{4},
$$

то последняя оценка имеет место всюду в плоскости, т.е.

$$
\left|\frac{d e}{d \bar{z}}(z) q(z) \exp \left(y_{0} z\right) f_{1}^{-1}(z) \theta^{-1}(z)\right| \leqslant \exp \left[H_{L_{1}}|z|\right] \quad \forall z .
$$

Выберем $\varepsilon^{\prime}>0$ такое, что

$$
H_{L_{1}}(z)+6 \varepsilon^{\prime}|z|<H_{G_{1}}(z) \quad \forall z \neq 0 .
$$

В силу (4.37) конечен интеграл

$$
\int_{\mathbb{C}}\left|q(z) \exp \left(y_{0} z\right) f_{1}^{-1}(z) \theta^{-1}(z) \frac{d e}{d \bar{z}}(z)\right|^{2} \exp \left(-2 H_{L_{1}}(z)-2 \varepsilon^{\prime}|z|\right) d \sigma .
$$

Тогда согласно теореме 4.4.2 из книги [17] найдется функция $\nu \in C^{\infty}$, являюшаяся решением $\bar{\partial}$-уравнения

$$
\frac{d \nu}{d \bar{z}}(z)=\frac{d e}{d \bar{z}}(z) q(z) \exp \left(y_{0} z\right) f_{i}^{-1}(z) \theta^{-1}(z)
$$

и удовлетворяюшая интегральной оценке

$$
\int_{\mathbb{C}}|\nu(z)|^{2} \exp \left(-2 H_{L_{1}}(z)-4 \varepsilon^{\prime}|z|\right) d \sigma<\infty
$$

Как и в предыдушей теореме, получаем

$$
\int_{\mathbb{C}}\left|\nu(z) f_{1}(z) \theta(z)\right|^{2} \exp \left(-2 H_{L_{1}}(z)-2 H_{K_{1}}(z)-8 \varepsilon^{\prime}|z|\right) d \sigma<\infty .
$$

В силу (4.33) и определения $q(z)$ получаем

$$
\left|e(z) q(z) \exp \left(y_{0} z\right)\right| \leqslant \exp \left(\left(A_{5}+\left|y_{0}\right|\right)|z|\right) \quad \forall z .
$$

Пусть $K=\overline{L_{1}+K_{1}+B\left(0,4 \varepsilon^{\prime}\right)}$ и $X=B\left(\eta_{0}, 3 r \delta\right) \cap \mathbb{S}$. Из (4.38) и равенства $D=G_{1}+K_{1}$ следует, что

$$
H_{K}(z)+2 \varepsilon^{\prime}|z|<H_{D}(z) \quad \forall z \neq 0 .
$$

Так как $X$ компактно принадлежит $I_{G}=I_{D}$, то по лемме 3.2 найдется компакт $L \subset D$ такой, что $K \subset L$ и выполнены неравенства

$$
\begin{gathered}
H_{L}(z)+2 \varepsilon^{\prime}|z|<H_{D}(z), \quad z \in \mathbb{C} \backslash\{0\}, \\
H_{L}(z)>\left(A_{5}+\left|y_{0}\right|\right)|z| \quad \forall z: z /|z| \in X .
\end{gathered}
$$


Согласно (4.40) с учетом вложения $K \subset L$ получаем

$$
\int_{\mathbb{C}}\left|\nu(z) f_{1}(z) \theta(z)\right|^{2} \exp \left(-2 H_{L}(z)\right) d \sigma<\infty .
$$

Поскольку $e(z)$ равна нулю вне угла, порожденного множеством $X$, то из (4.41) и (4.43) следует, что

$$
\int_{\mathbb{C}}\left|e(z) q(z) \exp \left(y_{0} z\right)\right|^{2} \exp \left(-2 H_{L}(z)-2 \varepsilon^{\prime}|z|\right) d \sigma<\infty .
$$

Отсюда с учетом (4.44) получаем интегральную оценку для функции $F(z)=e(z) \times$ $q(z) \exp \left(y_{0} z\right)-\nu(z) f_{i}(z) \theta(z)$ :

$$
\int_{\mathbb{C}}|F(z)|^{2} \exp \left(-2 H_{L}(z)-2 \varepsilon^{\prime}|z|\right) d \sigma<\infty .
$$

В силу (4.39) функция $F(z)$ целая. Пусть $T=\overline{L+B\left(0,2 \varepsilon^{\prime}\right)}$. Тогда из последнего неравенства (как и при получении оценки на $\varphi_{i}(z)$ в теореме 3.7 ) следует, что

$$
|F(z)| \leqslant C \exp H_{T}(z) \quad \forall z
$$

для некоторого $C>0$. В силу (4.42) компакт $T$ лежит в области $D$. Следовательно, согласно определению пространства $\mathbf{P}_{D}$ оно содержит функцию $F(z)$. Точно так же, как и в предыдущей теореме, показывается, что $F \in I(D, \Lambda)$. По условию система (1.1) разрешима в $H(D)$ для любой допустимой правой части из $H\left(G_{1}\right) \times \cdots \times H\left(G_{n}\right)$. Тогда по лемме 1.2 найдется элемент $\left(\varphi_{1}, \ldots, \varphi_{n}\right)$ пространства $\mathbf{P}_{G_{1}} \times \cdots \times \mathbf{P}_{G_{n}}$ такой, что

$$
F \equiv f_{1} \varphi_{1}+\cdots+f_{n} \varphi_{n}
$$

Используя (4.29) и проводя те же рассуждения, что и в теореме 4.4 , получаем

$$
\left|\frac{F\left(z_{m}\right)}{q_{3 r \delta}\left(z_{m}, z_{k_{m}}, \Lambda\right)}\right| \leqslant \exp \left(-B_{m}^{\prime}\left|z_{m}\right|\right), \quad m=1,2, \ldots,
$$

где $B_{m}^{\prime} \rightarrow+\infty$, когда $m \rightarrow \infty$. С другой стороны, с учетом $(4.35)$, как и в теореме 4.4, имеем

$$
\left|\frac{F\left(z_{m}\right)}{q_{3 r \delta}\left(z_{m}, z_{k_{m}}, \Lambda\right)}\right|=\left|\exp \left(y_{0} z_{m}\right)\right|=\exp \operatorname{Re}\left(y_{0} z_{m}\right)>1, \quad m \geqslant m_{4} .
$$

Полученное противоречие означает, что (4.28) верно, т.е. система $\left(f_{1}, \ldots, f_{n}\right)$ имеет слабый регулярный рост на луче $t \eta_{0}$. Теорема доказана.

ТЕОРема 4.6. Пусть $G$ - область с гладкой границей такая, что $I_{G}$ - открытое множество (возможно, пустое). Тогда для того, чтобы система (1.1) была разрешима в $H(D)$ для любой допустимой правой части из $H\left(G_{1}\right) \times \cdots \times H\left(G_{n}\right)$, необходимо и достаточно выполнение следуюших условий:

1) система функиий $\left(f_{1}, \ldots, f_{n}\right)$ имеет регулярный рост на луче каждого $\eta \in \mathbb{S} \backslash I_{G}$;

2) система функиий $\left(f_{1}, \ldots, f_{n}\right)$ имеет слабый регулярный рост на луче для каждого $\eta \in \mathbb{S} \cap I_{G}$.

ДокаЗАтЕльство. Достаточность следует из теоремы 3.7 и открытости множества $I_{G}$. Необходимость вытекает из предложения 4.3, теоремы 4.4 и того, что в условиях теоремы множество $\Gamma(G)$ совпадает с $\mathbb{C} \backslash I_{G}$. 


\section{Список литературы}

1. Левин Б. Я. Распределение корней целых функций. М.: Гостехиздат, 1956.

2. Напалков В. В. Уравнения свертки в многомерных пространствах. М.: Наука, 1982.

3. Кривошеев A.C. Критерий разрешимости неоднородных уравнений свертки в выпуклых областях пространства $\mathbb{C}^{n} / /$ Изв. АН СССР. Сер. матем. 1990. Т. 54 . №3. C. $480-500$.

4. Кривошеев A. C., Напалков B. В. Комплексный анализ и операторы свертки // УМН. 1992. T. 47. №6(288). C. 3-58.

5. Ehrenpreis L. Fourier analysis in several complex variables. N.Y.: Wiley-Interscience publishers, 1970.

6. Malgrange B. Systemes differentiells a coefficients constants. Seminaire Bourbaki, № 246 . Paris, 1962/63.

7. Напалков В. В. О системах неоднородных дифференциальных уравнений в частных производных бесконечного порядка // Матем. заметки. 1979. Т. 26. № 2. С. 217-226.

8. Дьедонне Ж., Швари Л. Двойственность в пространствах (F) и (LF) // Математика. 1958. T. 2. № 2. C. $77-107$.

9. Красичков-Терновский И. Ф. Инвариантные подпространства аналитических функций. І. Спектральный синтез на выпуклых областях // Матем. сб. 1972. Т. 87(129). № 4. C. 459-489.

10. Красичков-Терновский И. Ф. Инвариантные подпространства аналитических функций. II. Спектральный синтез на выпуклых областях // Матем. сб. 1972. Т. 88(130). № 1. C. 3-30.

11. Wiegerink J. J. Growth properties of Paley-Wiener functions on $\mathbb{C}^{n} / /$ Nederl. Akad. Wetensch. Proc. 1984. V. 87. P. 95-112.

12. Sigurdsson $R$. Convolution equations in domains of $\mathbb{C}^{n} / /$ Arkiv For Mat. 1991. V. 29. P. 285-305.

13. Хермандер Л. Анализ линейных дифференциальных операторов с частными производными. I. Теория распределений и анализ Фурье. М.: Мир, 1986.

14. Лейхтвейс K. Выпуклые множества. М.: Наука, 1985.

15. Леонтьев А. Ф. Целые функции. Ряды экспонент. М.: Наука, 1983.

16. Шабат Б. В. Введение в комплексный анализ. Ч.ІІІ. М.: Наука, 1985.

17. Хермандер Л. Введение в теорию функций нескольких комплексных переменных. М.: Мир, 1968.

18. Лелон П., Груман Л. Целые функции многих комплексных переменных. М.: Мир, 1989.

Поступило в редакцию

13.IV.1999 